\title{
FREE DIVISION RINGS OF FRACTIONS OF CROSSED PRODUCTS OF GROUPS WITH CONRADIAN LEFT-ORDERS
}

\author{
JOACHIM GRÄTER
}

\begin{abstract}
Let $D$ be a division ring of fractions of a crossed product $F[G, \eta, \alpha]$ where $F$ is a skew field and $G$ is a group with Conradian left-order $\leq$. For $D$ we introduce the notion of freeness with respect to $\leq$ and show that $D$ is free in this sense if and only if $D$ can canonically be embedded into the endomorphism ring of the right $F$-vector space $F((G))$ of all formal power series in $G$ over $F$ with respect to $\leq$. From this we obtain that all division rings of fractions of $F[G, \eta, \alpha]$ which are free with respect to at least one Conradian left-order of $G$ are isomorphic and that they are free with respect to any Conradian left-order of $G$. Moreover, $F[G, \eta, \alpha]$ possesses a division ring of fraction which is free in this sense if and only if the rational closure of $F[G, \eta, \alpha]$ in the endomorphism ring of the corresponding right $F$-vector space $F((G))$ is a skew field.
\end{abstract}

\section{INTRODUCTION}

This paper deals with crossed products $F[G, \eta, \alpha]$ of a group $G$ with Conradian leftorder $\leq$ over a skew field $F$. We are mainly interested in the case when $F[G, \eta, \alpha]$ possesses a division ring of fractions $D$. By this we mean a skew field $D$ which contains $F[G, \eta, \alpha]$ as a subring such that $D$ is generated by $F[G, \eta, \alpha]$ as a division ring. In Section 5 we introduce the notion of freeness with respect to a Conradian left-order $\leq$ of $G$. Our first main result states that if $D$ meets this freeness condition then any non-zero $x \in D$ which is not a unit in $F[G, \eta, \alpha]$ possesses a "unique" normal form representation which can roughly be described as a local formal power series expansion over an archimedean ordered group where the coefficients are stricly simpler than $x$ with respect to the complexity discussed in [D2, D3, DGH]. The archimedean ordered group corresponds to a factor group $C / C^{\prime}$ where $\left(C^{\prime}, C\right)$ is a convex jump of $G$ with respect to the Conradian left-order $\leq$ and the coefficients are elements of the rational closure of $F\left[C^{\prime}, \eta, \alpha\right]$ in $D$. From this we derive our next main result that any element of $D$ can be comprehended as an endomorphism of the right $F$-vector space $F((G))$ of all formal power series in $G$ over $F$. In order to achieve this we first consider $F[G, \eta, \alpha]$ as a subring of the endomorphism ring $\operatorname{End}(F((G)))$ where the elements of $F[G, \eta, \alpha]$ act on $F((G))$ via multiplication from the left. Then Theorem 8.1 states that $D$ is isomorphic to the rational closure $F(G, \eta, \alpha)$ of $F[G, \eta, \alpha]$ in $\operatorname{End}(F((G)))$. The following results are direct conclusions of our main theorems where the assumptions are as above. The rational closure $F(G, \eta, \alpha)$ of $F[G, \eta, \alpha]$ is a division ring if and only if $F[G, \eta, \alpha]$

2010 Mathematics Subject Classification. 16S35, 16S34, 20F60, 16S85, 16W60, 12 E15.

Key words. Crossed product, group ring, ordered group, Conradian left-order, locally indicable group, division ring of fractions, Hughes-free, formal power series.

Date: October 17, 2019 
possesses at least one division ring of fractions which is free with respect to $\leq$. In this case $F(G, \eta, \alpha)$ itself is free with respect to $\leq$, all such division rings of fractions are isomorphic to $F(G, \eta, \alpha)$, and every automorphisms of $F[G, \eta, \alpha]$ can be extended to an automorphism of $F(G, \eta, \alpha)$. For the division ring $D$ there are also other definitions of freeness which are due to I. Hughes and P.A. Linnell since any group with Conradian left-order is locally indicable and vice versa. In Section 8 we will study the relation between all these concepts of freeness and finally Section 9 contains a discussion of Hughes' two main theorems from [H1, $\mathrm{H} 2$ ] in the light of our results.

The topic of this paper is closely related to what has been investigated in [J] where, however, the results are restricted to the special class of Conradian left-orders with maximal rank. Our approach does not require any further assumption. The theorems we prove hold true for all Conradian left-orders and they include the results from [J] in this extended and generalized form.

Throughout our investigation we make intensive use of two concepts that have originally been introduced by N.I. Dubrovin. Firstly, there is the complexity $c p(x)$ of an element $x$ in $D$ we already mentioned above. It is an ordinal which describes how $x$ is built from elements of $F[G, \eta, \alpha]$ by addition, multiplication, and the invers operation. It enables us to prove statements about the elements of $D$ by transfinite induction. Section 4 provides all necessary background information which is needed to apply this concept. The proof of Theorem 8.1 also requires Dubrovin's remarkable work on $v$-compatible and continuous endomorphisms of the right $F$-vector space $F((G))$ as given in [D2, D3].

\section{Conradian Groups}

In order to make this paper self-contained we present in this short section all of the basics of left-ordered groups which will be used in the following. There will be nothing really new for the specialists but it hopefully improves the readability. A group $G$ equipped with a linear order $\leq$ is called left-ordered (with respect to $\leq$ ) if $a \leq b$ implies $c a \leq c b$ for all $a, b, c \in G$ and we also say that $\leq$ is a left-order of $G$. Rightorders are defined similarly but in this paper they are only needed on rare occasions. If they occur it will always be clear how to adopt the corresponding definitions and results stated previously for left-orders. For any left-order $\leq$ of $G$ the set $P_{\leq}=\{a \in G \mid e \leq a\}$ is called the positive cone of $\mathrm{G}$ with respect to $\leq$, where $e$ denotes the unit-element of $G$. Since $a \leq b \Longleftrightarrow a^{-1} b \in P_{\leq}$for all $a, b \in G$ the left-order $\leq$ is uniquely determined by $P_{\leq}$. It is easily seen that $P_{\leq} \cup P_{\leq}^{-1}=G, P_{\leq} \cap P_{\leq}^{-1}=\{e\}$, and $P_{\leq} P_{\leq} \subseteq P_{\leq}$where $A^{-1}=\left\{a^{-1} \mid a \in A\right\}$ and $A B=\{a b \mid a \in A, b \in B\}$ for arbitrary $A, B \subseteq G$ as usual. On the other hand if $P$ is a subset of $G$ satisfying $P \cup P^{-1}=G, P \cap P^{-1}=\{e\}$, and $P P \subseteq P$ then $a \leq^{\prime} b \Longleftrightarrow a^{-1} b \in P$ for all $a, b \in G$ defines a left-order of $G$ such that $P$ is the corresponding positive cone. If $U$ is a subgroup of $G$ then any left-order $\leq$ of $G$ induces a left-order of $U$ (also denoted by $\leq$ ) and we will always consider $U$ as a left-ordered group in this way unless otherwise stated. For groups $G$ and $G^{\prime}$ with left-orders $\leq$ and $\leq^{\prime}$ respectively a group homomorphism $\varphi: G \longrightarrow G^{\prime}$ is said to be order-preserving or an $o$-homomorphism if $a \leq b \Longrightarrow \varphi(a) \leq^{\prime} \varphi(b)$ for all $a, b \in G$. We call $G$ and $G^{\prime}$ order-isomorphic with respect to $\leq$ and $\leq$ if there 
exists a group isomorphism $\varphi: G \longrightarrow G^{\prime}$ which is order-preserving. The kernel of an $o$-homomorphism $\varphi: G \longrightarrow G^{\prime}$ is a convex normal subgroup of $G$. On the other hand, if $N$ is a convex normal subgroup of $G$ then $P=\left\{a N \mid a \in P_{\leq}\right\}$is a positive cone of the factor group $G / N$. The corresponding left-order $\leq^{\prime}$ of $G / N$ is called the canonical left-order of $G / N$ with respect to $\leq$ and $\varphi: G \longrightarrow G / N$ is an $o$-homomorphism.

Proposition 2.1. (cf. [C]) Let $G$ and $G^{\prime}$ be groups with left-orders $\leq$ and $\leq$ respectively. If $\varphi: G \longrightarrow G^{\prime}$ is a surjective o-homomorphism then the correspondence $C \longmapsto \varphi(C)$ induces an inclusion-preserving bijection between the set of all convex subgroups of $G$ containing the kernel of $\varphi$ and the set of all convex subgroups of $G^{\prime}$.

Definition 2.2. Let $G$ be a group and let $\leq$ be a left-order of $G$. Then $G$ is called archimedean left-ordered and $\leq$ an archimedean left-order of $G$ if for all $a, b \in G, e<b$ there is an $n \in \mathbb{N}$ such that $a<b^{n}$.

The following result is a generalization of the classical Hölder theorem (cf. [Hö,, $\mathrm{C}]$ ):

Theorem 2.3. Every archimedean left-ordered group is order-isomorphic to a subgroup of the naturally ordered additive group $\mathbb{R}$. Especially, any archimedean left-ordered group is commutative and possesses no proper convex subgroup.

Let $\leq$ be a left-order of the group $G$ and let $\mathcal{C}_{\leq}$denote the set of all convex subgroups of $G$. Then $\mathcal{C}_{\leq}$is a linearly ordered complete set with respect to the inclusion containing $\{e\}$ and $G$. A pair $\left(C^{\prime}, C\right)$ is called a convex jump in $G$ (with respect to $\leq$ ) whenever $C^{\prime}, C \in \mathcal{C}_{\leq}$and $C^{\prime}$ is a lower neighbour of $C$, that is, $C^{\prime}$ is a proper subgroup of $C$ and there is no convex subgroup of $G$ stricly between $C^{\prime}$ and $C$ (cf. [C, $\left.\mathrm{KM}\right]$ ).

Definition 2.4. Let $G$ be a left-ordered group with respect to $\leq$ and let $g$ be an arbitrary element of $G$. Then $C_{g}^{+}$denotes the minimal convex subgroup of $G$ containing $g$. If $g$ is non-trivial then $C_{g}^{-}$denotes the maximal convex subgroup of $G$ which does not contain $g$.

Cleary, $\left(C_{g}^{-}, C_{g}^{+}\right)$is a convex jump in $G$ for all non-trivial $g$ in $G$. On the other hand if $\left(C^{\prime}, C\right)$ is a convex jump in $G$ then $C^{\prime}=C_{g}^{-}$and $C=C_{g}^{+}$for any $g \in C$ which does not lie in $C^{\prime}$. If in addition $C^{\prime}$ is a normal subgroup in $C$ then the factor group $C / C^{\prime}$ is called a factor of $\mathcal{C}_{\leq}$.

Definition 2.5. (cf. $[\mathrm{C}, \overline{\mathrm{KM}}]$ ). Let $G$ be a left-ordered group with respect to $\leq$. Then $G$ and $\leq$ are said to be Conradian if for any convex jump $\left(C^{\prime}, C\right)$ in $G$ with respect to $\leq$ the convex subgroup $C^{\prime}$ is normal in $C$ such that the factor $C / C^{\prime}$ is archimedean ordered with respect to the canonical order.

Conradian left-orders can also be characterized without using convex subgroups (cf. $[\mathrm{C}, \mathrm{KM}]$ ). In this respect it is worth mentioning that unlike us [C] and [KM] deal with right-orders (cf. [GS, Section 2]).

Proposition 2.6. Let $G$ be a group with left-order $\leq$. Then the following statements are equivalent:

(1) The left-order $\leq$ is Conradian.

(2) For all $a, b \in G$ such that $e<a, b$ there is an $n \in \mathbb{N}$ satisfying $a b<(b a)^{n}$.

(3) For all $a, b \in G$ such that $e<a<b$ there is an $n \in \mathbb{N}$ satisfying $b<a^{-1} b^{n} a$. 
(4) For all $a, b \in G$ such that $e<a, b$ there is an $n \in \mathbb{N}$ satisfying $a<b a^{n}$.

As a consequence of this last result we obtain the first part of the following

Proposition 2.7. Let $G$ be a group with Conradian left-order $\leq$ and let $U$ be a subgroup of $G$. Then the left-order of $U$ induced by $\leq$ is Conradian. Moreover, if $\left(C^{\prime}, C\right)$ is a convex jump in $U$ then there exists a convex jump $\left(\bar{C}^{\prime}, \bar{C}\right)$ in $G$ extending $\left(C^{\prime}, C\right)$, that is, $\bar{C}^{\prime} \cap U=C^{\prime}$ and $\bar{C} \cap U=C$. Especially, $C / C^{\prime}$ is isomorphic to a subgroup of $\bar{C} / \bar{C}^{\prime}$.

Proof. Clearly, $U$ is Conradian because of Proposition 2.6. Thus, let $\left(C^{\prime}, C\right)$ be a convex jump in $U$, let $\bar{C}^{\prime}$ be the maximal convex subgroup of $G$ such that $\bar{C}^{\prime} \cap U \subseteq C^{\prime}$ and let $\bar{C}$ be the minimal convex subgroup of $G$ containing $C$. Obviously, $\bar{C}^{\prime} \cap U$ and $\bar{C} \cap U$ are convex subgroups of $U$ and $\left(\bar{C}^{\prime}, \bar{C}\right)$ is a convex jump in $G$. The canonical left-order of $\bar{C} / \bar{C}^{\prime}$ induces on $(\bar{C} \cap U) /\left(\bar{C}^{\prime} \cap U\right)$ the canonical left-order. Therefore, $C /\left(\bar{C}^{\prime} \cap U\right)$ and $C^{\prime} /\left(\bar{C}^{\prime} \cap U\right)$ are two different convex subgroups of the archimedean ordered group $(\bar{C} \cap U) /\left(\bar{C}^{\prime} \cap U\right)$ by Proposition 2.1. Because of Theorem 2.3 we conclude $\bar{C}^{\prime} \cap U=C^{\prime}$ and $\bar{C} \cap U=C$.

If $\leq$ is a Conradian left-order then Theorem 2.3 shows that any factor of $\mathcal{C}_{\leq}$is orderisomorphic to a subgroup of the additive group $\mathbb{R}$.

Definition 2.8. A Conradian left-order of a group $G$ has maximal rank if any factor of $\mathcal{C}_{\leq}$is order-isomorphic to a subgroup of the naturally ordered additive group $\mathbb{Q}$.

This definition of maximality is equivalent to the one introduced in [J].

Proposition 2.9. Any torsion-free abelian group $G$ possesses an order $\leq$ with maximal rank such that $\mathcal{C}_{\leq}$is well-ordered with respect to the inclusion.

Proof. To simplify notation, let $G$ be written additively. We first assume that $G$ is divisible and regard $G$ as a $\mathbb{Q}$-vector space with basis $B=\left\{g_{i} \mid i \in I\right\}$ where $I$ is endowed with a well-order $\leq_{I}$. Any non-zero $a \in G$ can uniquely be written as $a=q_{i_{1}} g_{i_{1}}+\cdots+q_{i_{n}} g_{i_{n}}$ with $n \in \mathbb{N}, i_{1}<_{I} i_{2}<_{I} \cdots<_{I} i_{n}$, and non-zero rational numbers $q_{i_{1}}, \ldots, q_{i_{n}}$. Defining $i_{a}:=i_{n} \in I$ and $q(a):=q_{i_{n}} \in \mathbb{Q}$ it is easily seen that $P=\{a \in G \mid a=0$ or $0<q(a)\}$ is a positive cone in $G$ with respect to an order $\leq$ of $G$ such that for any $i \in I$

$$
H_{i}^{-}=\left\{a \in G \mid a=0 \text { or } i_{a}<i\right\}, H_{i}^{+}=\left\{a \in G \mid a=0 \text { or } i_{a} \leq i\right\}
$$

are convex subgroups of $G$ such that $H_{i}^{+} / H_{i}^{-}$and $\mathbb{Q}$ are isomorphic as ordered groups. Moreover, $H_{i}^{+} \subset H_{j}^{+}$for all $i, j \in I, i<_{I} j$ and $H_{i(a)}^{+}=C_{a}^{+}, H_{i(a)}^{-}=C_{a}^{-}$for all non-zero $a \in G$. This completes the proof if $G$ is divisible and we turn to the general situation. We consider the divisible hull $G^{\prime}$ of $G$ for which the proposition has been proved and apply Proposition 2.7.

Proposition 2.10. Let $G$ be a group with Conradian left-order $\leq$. Then there exists a Conradian left-order $\leq^{\prime}$ with maximal rank such that $\mathcal{C}_{\leq} \subseteq \mathcal{C}_{\leq^{\prime}}$. If $\mathcal{C}_{\leq}$is well-ordered with respect to the inclusion then $\leq^{\prime}$ can be choosen that $\mathcal{C}_{\leq^{\prime}}$ is also well-ordered. 
Proof. If $\left(C^{\prime}, C\right)$ is a convex jump in $G$ with respect to $\leq$ then Proposition 2.9 shows that there exists a left-order $\leq_{\left(C^{\prime}, C\right)}$ of $C / C^{\prime}$ with maximal rank such that the corresponding convex subgroups are well-ordered with respect to the inclusion. Now, $\mathcal{C}_{\leq}$is a linearly ordered, complete, and subnormal system of subgroups of $G$ with respect to the inclusion and we can apply [KM, Proposition 3.2.1]. Thus, there exists a left-order $\leq^{\prime}$ of $G$ such that $\mathcal{C}_{\leq} \subseteq \mathcal{C}_{\leq \prime}$ and for any convex jump $\left(C^{\prime}, C\right)$ in $G$ with respect to $\leq$ the canonical left-order of $C / C^{\prime}$ with respect to $\leq^{\prime}$ coincides with $\leq_{\left(C^{\prime}, C\right)}$. If $\left(\bar{C}^{\prime}, \bar{C}\right)$ is a convex jump in $G$ with respect to $\leq^{\prime}$ then there is a convex jump $\left(C^{\prime}, C\right)$ in $G$ with respect to $\leq$ satisfying $C^{\prime} \subseteq \bar{C}^{\prime} \subset \bar{C} \subseteq C$ and $\bar{C}^{\prime}$ is normal in $\bar{C}$. We conclude by Proposition 2.1 that $\left(\bar{C}^{\prime} / C^{\prime}, \bar{C} / C^{\prime}\right)$ is a convex jump in $C / C^{\prime}$ with respect to $\leq_{\left(C^{\prime}, C\right)}$ and therefore $\bar{C} / \bar{C}^{\prime}$ is order-isomorphic to a subgroup of the additive group $\mathbb{Q}$. Finally, if $\mathcal{C}_{\leq}$well-ordered and if $C_{1} \supset C_{2} \supset \ldots$ is a strictly decreasing chain of convex subgroups of $G$ with respect to $\leq^{\prime}$ then there exists a convex jump $\left(C^{\prime}, C\right)$ of $G$ with respect to $\leq$ such that $C \supseteq C_{k} \supset C_{k+1} \supset \cdots \supset C^{\prime}$ for some $k \in \mathbb{N}$ since $\mathcal{C}_{\leq}$is wellordered. But then $C / C^{\prime} \supseteq C_{k} / C^{\prime} \supset C_{k+1} / C^{\prime} \supset \cdots \supset C^{\prime} / C^{\prime}$ is a strictly decreasing chain of convex subgroups of $C / C^{\prime}$ with respect to $\leq_{\left(C^{\prime}, C\right)}$ which is a contradiction.

G] provides a more detailed account of the results above and their proofs. Conradian left-orders can also be characterized by purely group-theoretic properties, that is, without mentioning terms from order theory.

Definition 2.11. Let $G$ be a group. Then $G$ is said to be indicable if there exists a surjective group homomorphism $\varphi: G \longrightarrow \mathbb{Z}$. And $G$ is called locally indicable whenever any non-trivial finitely generated subgroup of $G$ is indicable.

According to the definition above, a group $G$ is indicable if and only if $G$ possesses a normal subgroup $N$ such that the factor group $G / N$ is an infinite cyclic group. Locally indicable groups have been introduced by Graham Higman in [Hi] where he investigated zero-divisors and units in certain group rings. They also form an important class of groups in connection with the solvability of equations over groups (cf. $[\mathrm{Br}]$ ) and they occur naturally and in different ways in topology and geometry as fundamental groups of surfaces and manifolds, for instance as knot groups of classical knots. (cf. [CR]).

Theorem 2.12. Any Conradian group is locally indicable.

A more general version of this theorem has been proved by R.G. Burns and V.W. Hale and later on also by other authors (cf. $[\mathrm{BH}]$ ). A nice proof of Theorem 2.12 is given in [CR, Section 10.1] which can be sketched as follows. Let $G=\left\langle g_{1}, \ldots, g_{n}\right\rangle$ be a non-trivial finitely generated subgroup of a Conradian group. By Proposition 2.7 the group $G$ is also Conradian and there exists $g \in\left\{g_{1}, \ldots, g_{n}\right\}$ such that $G=C_{g}^{+}$. Then $N:=C_{g}^{-}$is normal in $G$ and $G / N$ is a non-trivial, finitely generated, and torsion free abelian group. Thus, $G \cong \oplus_{i=1}^{k} \mathbb{Z}$ for some $k \in \mathbb{N}$ and now it is clear how to obtain a surjective group homomorphism $\varphi: G \longrightarrow G / N \longrightarrow \mathbb{Z}$.

Even the convers of Theorem 2.12 holds true:

Theorem 2.13. Any locally indicable group is Conradian. 
Unlike Theorem 2.12 a proof of Theorem 2.13 requires much more effort (cf. $\overline{\mathrm{Br}}$, $\overline{\mathrm{RR}}$, N]). The fact that the class of all Conradian groups coincides with the class of all locally indicable groups is of particular interest for this paper.

\section{Crossed Products}

In what follows, all rings are associative but not necessarily commutative, with unit element $1 \neq 0$ which is preserved by homomorphisms and inherited by subrings. If $R$ is a ring then we write $R^{\times}$for the multiplicative group of units of $R$ and $R$ is called an integral domain if $R$ contains no non-trivial zero-divisor. By a skew field or division ring we shall understand a ring in which any non-zero element is a unit. In this section we summarize all definitions, propositions, and theorems about crossed products which are needed in this paper. We mainly refer to Section 1.1 of D.S. Passman's book Infinite Crossed Products (cf. $[\mathrm{Pa}]$ ).

Let $R$ be a ring and $G$ a group. A crossed product of $G$ over $R$ is a ring $S$ containing $R$ as a subring which turns $S$ naturally into a left $R$-module which is free with basis $X_{G}=\left\{x_{g} \mid g \in G\right\}$ where $x_{g} \neq x_{h}$ if $g \neq h$ for all $g, h \in G$. By this we mean that the additive structure of $S$ as a ring coincides with the additive structure of $S$ as an $R$ module and with respect to the multiplication there are two mappings $\eta: G \times G \longrightarrow R^{\times}$ and $\alpha: G \longrightarrow$ Aut $R, g \longmapsto \alpha_{g}$ having the following properties:

(1) $1 x_{e}$ is the unit element of $S$,

(2) $a x_{e} \cdot b x_{e}=a b x_{e}$ for all $a, b \in R$,

(3) $a x_{e} \cdot 1 x_{g}=a x_{g}$ for all $a \in R$ and $g \in G$,

(4) $1 x_{g} \cdot 1 x_{h}=\eta(g, h) x_{g h}$ for all $g, h \in G$,

(5) $1 x_{g} \cdot a x_{e}=\alpha_{g}(a) x_{g}$ for all $a \in R$ and $g \in G$.

From this we obtain the following equations for all $g, h, l \in G$ and $a \in R$ :

(6) $\eta(g, e)=\eta(e, g)=1$,

(7) $\alpha_{g} \alpha_{h}(a)=\eta(g, h) \alpha_{g h}(a) \eta(g, h)^{-1}$,

(8) $\alpha_{g}(\eta(h, l)) \eta(g, h l)=\eta(g, h) \eta(g h, l)$.

Because of (1) and (2) we usually write $a$ instead of $a x_{e}$ for all $a \in R$ and $x_{g}$ instead of $1 x_{g}$ for all $g \in G$ such that (3) provides $a \cdot x_{g}=a x_{e} \cdot 1 x_{g}=a x_{g}$. Then any element $x \in S$ can uniquely be written as a finite sum $x=\sum a_{g} x_{g}$ with $a_{g} \in R, g \in G$ and the support of $x$ is defined as the set of all $g \in G$ with non-zero $a_{g}$. Moreover, the multiplication in $S$ turns into the following form:

$$
\left(\sum a_{g} x_{g}\right) \cdot\left(\sum b_{h} x_{h}\right)=\sum a_{g} x_{g} \cdot b_{h} x_{h} \text { where } a_{g} x_{g} \cdot b_{h} x_{h}=a_{g} \alpha_{g}\left(b_{h}\right) \eta(g, h) x_{g h} .
$$

This shows that the structure of $S$ as a ring and as an $R$-module is uniquely determined by $R, G, \eta$, and $\alpha$ and therefore we also write $R[G, \eta, \alpha]$ instead of $S$.

On the other hand if a ring $R$ and a group $G$ are given together with two mappings $\eta: G \times G \longrightarrow R^{\times}$and $\alpha: G \longrightarrow$ Aut $R, g \longmapsto \alpha_{g}$ such that (6),(7), and (8) are satisfied then there exists a crossed product $S=R[G, \eta, \alpha]$ of $G$ over $R$.

Any $x_{g}, g \in G$ is a unit in $R[G, \eta, \alpha]$ such that $x_{g} a x_{g}^{-1}=\alpha_{g}(a)$ for all $a \in R$. This shows that the conjugation by $x_{g}$ defines a ring automorphism of $R[G, \eta, \alpha]$ which extends $\alpha_{g}$ 
to $R[G, \eta, \alpha]$ and which will be denoted by $\alpha_{g}$ again, that is,

$$
\alpha_{g}\left(\sum a_{h} x_{h}\right)=\sum \alpha_{g}\left(a_{h}\right) x_{g} x_{h} x_{g}^{-1} .
$$

If $T$ is a subring of $R$ and $U$ a subgroup of $G$ such that $\alpha_{g}(T)=T$ for all $g \in U$ and and $\eta(g, h) \in T^{\times}$for all $g, h \in U$ then the subring of $R[G, \eta, \alpha]$ which is generated by $T$ and $X_{U}$ is a crossed product of $U$ over $T$ in an obvious way. By abuse of notation, this ring will also be denoted by $T[U, \eta, \alpha]$. The proof of the following proposition is straightforward.

Proposition 3.1. With the notation as above the following holds true: If $N$ is a normal subgroup of $G$ and if $\mathfrak{G}$ is a transversal for $N$ in $G$ such that $e \in \mathfrak{G}$ then $R[G, \eta, \alpha]$ is a crossed product of $G / N$ over $R[N, \eta, \alpha]$ with basis $X_{G / N}=\left\{x_{g} \mid g \in \mathfrak{G}\right\}$. Moreover, for any $g \in G$ the restriction $\bar{\alpha}_{g}$ of $\alpha_{g}$ to $R[N, \eta, \alpha]$ is a ring automorphism of $R[N, \eta, \alpha]$.

If $R[G, \eta, \alpha]$ is a crossed product of $G$ over $R$ and $S$ a $\operatorname{ring}$ containing $R$ as a subring such that any $\alpha_{g}, g \in G$ can be extended uniquely to a ring automorphism $\bar{\alpha}_{g}$ of $S$ then conditions $(6)-(8)$ apply accordingly for $\eta$ and $\bar{\alpha}: G \longrightarrow$ Aut $S, g \longmapsto \bar{\alpha}_{g}$. This is rather obvious for property (6) and (8). In order to prove property (7) we first observe that the automorphism $\alpha_{h}$ can be extended to $S$ by $\bar{\alpha}_{h}$ on the one hand, and by the automorphism of $S$ arising from the composition of $\bar{\alpha}_{g h}$, the conjugation by $\eta(g, h)$, and $\bar{\alpha}_{g}^{-1}$ on the other. Because of the uniqueness of the extension we are done. Thus we have proved

Proposition 3.2. With the notation as above the following holds true: Let $R$ be a subring of the ring $S$ and let $R[G, \eta, \alpha]$ be a crossed product of $G$ over $R$. If for any $g \in G$ there exists a unique ring automorphism $\bar{\alpha}_{g}$ of $S$ extending $\alpha_{g}$ then $R[G, \eta, \alpha]$ coincides in an obvious way with the subring of $S[G, \eta, \bar{\alpha}]$ which is generated by $R$ and $X_{G}$.

The following proposition has been used before by other authors in different variants. For the sake of completeness, we will briefly outline a proof for crossed products of groups which are left-ordered (cf. [Bo]).

Proposition 3.3. Let $R=F[G, \eta, \alpha]$ be a crossed product of a group $G$ with left-order $\leq$ over a skew field $F$. Then $R$ is an integral domain with group of units $F^{\times} X_{G}$. If $\varphi: R \longrightarrow R$ is a ring automorphism then $\varphi(F)=F, \varphi\left(F X_{G}\right)=F X_{G}$, and $R$ is a crossed product of $G$ over $F$ with respect to the basis $X_{G}^{\prime}=\left\{x_{g}^{\prime} \mid g \in G\right\}$ where $x_{g}^{\prime}=\varphi\left(x_{g}\right)$ for all $g \in G$.

Proof. Let $x, y$ be non-zero elements in $R$ where $x=a_{1} x_{g_{1}}+\cdots+a_{m} x_{g_{m}}$ and $y=$ $b_{1} x_{h_{1}}+\cdots+b_{n} x_{h_{n}}$ with $a_{1}, \ldots, a_{m}, b_{1}, \ldots, b_{n} \in F^{\times}$. We assume $g_{1}<g_{2}<\cdots<g_{m}$ and $h_{1}<h_{2}<\cdots<h_{n}$. Then $g_{i} h_{1}<\cdots<g_{i} h_{n}$ follows for all $i \in\{1, \ldots, m\}$. Let $k$ be in $\{1, \ldots, m\}$ such that $g_{k} h_{n} \geq g_{1} h_{n}, \ldots, g_{m} h_{n}$. We conclude

$$
g_{k} h_{n}>g_{i} h_{j} \text { for }(i, j) \neq(k, n) .
$$

Therefore, $a_{k} \alpha_{g_{k}}\left(b_{n}\right) \eta\left(g_{k}, h_{n}\right)$ is the non-zero coefficient of $g_{k} h_{n}$ in the presentation of $x y$ which proves the first claim of the proposition. By similar arguments there exists $l \in\{1, \ldots, m\}$ such that

$$
g_{l} h_{1}<g_{i} h_{j} \text { for }(i, j) \neq(l, 1)
$$


and $g_{l} h_{1}$ is also an element of the support of $x y$. Now, let $x$ be a unit in $R$ and let $x y=1$. In order to show $x \in F^{\times} X_{G}$ we assume $1<m$. Then there exists an $i \in\{1, \ldots, m\}$ different from $l$ and $g_{l} h_{1}<g_{i} h_{1} \leq g_{k} h_{n}$ follows. This shows that the support of $x y$ contains more than one element which contradicts $x y=1$. Since any element of $F^{\times} X_{G}$ is obviously a unit in $R$ the second claim of the proposition is shown. Now, let $\varphi: R \longrightarrow R$ be a ring automorphism. Since $F^{\times} X_{G}$ is the group of units in $R$ we obtain $\varphi\left(F^{\times} X_{G}\right)=F^{\times} X_{G}$, that is $\varphi\left(F X_{G}\right)=F X_{G}$. Next we prove $\varphi(F) \subseteq F$. Then $\varphi^{-1}(F) \subseteq F$ follows and we get $\varphi(F)=F$ as claimed. Let $a$ be in $F$ different from 0 and 1. Since $a-1$ is a unit in $R$ there exist $b \in F^{\times}$and $g \in G$ such that $\varphi(a)=b x_{g}$. Hence, $\varphi(a-1)=\varphi(a)-\varphi(1)=b x_{g}-x_{e} \in F^{\times} X_{G}$ which shows $g=e$. The claim that $R$ is a crossed product of $G$ over $F$ with respect to the basis $X_{G}^{\prime}$ can now be derived by straightforward computation.

Before presenting the last result of this section we discuss two examples where $R$ is assumed to be a (left and right) Ore domain (cf. [Co, Section 5]). Firstly, let $S=R[\langle g\rangle, \eta, \alpha]$ be a crossed product of an infinite cyclic group $G=\langle g\rangle$ over $R$. For any $n \in \mathbb{Z}$ there exists a unit $a_{n} \in R^{\times}$such that $x_{g^{n}}=a_{n} x_{g}^{n}$. Thus $\left\{x_{g}^{n} \mid n \in \mathbb{Z}\right\}$ is an $R$-basis of the free $R$-module $S$, that is, $S$ can be seen as the skew Laurent polynomial ring in $x_{g}$ over $R$ which shows that $R[\langle g\rangle, \eta, \alpha]$ is an Ore domain. In the second example $G$ is isomorphic to a subgroup of the additive group $\mathbb{Q}$. For given $a, b \in S$ there exist $g, g_{1}, \ldots, g_{n} \in G$ such that $a, b \in R x_{g_{1}}+\cdots+R x_{g_{n}}$ and $\left\langle g_{1}, \ldots, g_{n}\right\rangle=\langle g\rangle$. Therefore, $a, b$ belong to the subring of $S$ generated by $R, x_{g}$, and $x_{g^{-1}}$, that is, $a, b \in R[\langle g\rangle, \eta, \alpha]$ which is an Ore domain as mentioned above. Thus, $R[G, \eta, \alpha]$ is an Ore domain too.

Proposition 3.4. Let $R=F[G, \eta, \alpha]$ be a crossed product of a group $G$ over a skew field $F$. If $G$ possesses a Conradian left-order $\leq$ such that $\mathcal{C}_{\leq}$is well-ordered with respect to the inclusion then $R$ is an Ore domain.

Proof. Because of Proposition [2.10 let $\leq$ be a Conradian left-order with maximal rank. We assume that $F[G, \eta, \alpha]$ is not an Ore domain and choose $C \in \mathcal{C}_{\leq}$minimal with respect to the inclusion such that $F[C, \eta, \alpha]$ is not an Ore domain. Then $C$ must have a lower neighbour $C^{\prime} \in \mathcal{C}_{\leq}$, that is, $\left(C^{\prime}, C\right)$ is a convex jump. According to Proposition [3.1, $F[C, \eta, \alpha]$ is a crossed product of $C / C^{\prime}$ over $F\left[C^{\prime}, \eta, \alpha\right]$. Because of the minimality of $C$ we conclude that $F\left[C^{\prime}, \eta, \alpha\right]$ is an Ore domain. Since $C / C^{\prime}$ is isomorphic to a subgroup of the additive group $\mathbb{Q}$ the second example above provides the desired contradiction.

\section{The Complexity}

If $D$ is a division ring with subring $R$ then the rational closure of $R$ in $D$ is the minimal subdivision ring of $D$ containing $R$. If $D$ itself is this rational closure then $D$ is called a division ring of fractions of $R$. In this case any element $d$ of $D$ is built from elements of $R$ by means of addition, multiplication, and the invers operation, that is, $d$ possesses a presentation as a rational expression of elements from $R$. Of course, such a presentation 
is not unique and in general it does not even exist a unique normal form in which any presentation can be transformed. Despite this difficulty it is shown in [DGH] that it is always possible to assign ordinals to the elements of a division ring of fractions in order to describe the complexity of the expressions which are needed to present an element of $D$ by elements from $R$. For $d \in D$ this ordinal is called the complexity of $d$ denoted by $c p(d)$. The complexity allows us to prove statements about the elements of $D$ by transfinite induction which is an essential tool for proving our main theorems. In this section we give a short introduction into the basic concepts of the complexity and we explain how to apply the results from [DGH]. We also present some results which are not included in [DGH] but which are needed here.

In this section $T$ always denotes a ring (which is denoted by $R$ in [DGH]). If $A$ is a subset of $T$ then $A^{-1}$ consists of all $a^{-1}$ where $a \in A \cap T^{\times}$. By the rational closure of $A$ in $T$ we will understand the smallest subring $D$ of $T$ which contains $A$ such that $D^{-1} \subseteq D$. We fix a subset $\mathcal{G}$ of $T^{\times}$(which is denoted by $S$ in [DGH]) and assume that $\mathcal{G}$ is a subgroup of $T^{\times}$and $-g \in \mathcal{G}$ for all $g \in \mathcal{G}$, that is $-\mathcal{G}=\mathcal{G}$. Finally, $D$ denotes the rational closure of $\mathcal{G}$ in $T$. In our applications $T$ always contains a subring $R=F[G, \eta, \alpha]$ which is a crossed product of a left-ordered group $G$ over a skew field $F$. Then $\mathcal{G}$ will be the group of units of $R$, that is, $\mathcal{G}=F^{\times} X_{G}$ and $R$ has no non-trivial zero-divisor. If $D$ is a division ring then $D$ is a division ring of fractions of $F[G, \eta, \alpha]$.

As mentioned above any $a \in D$ is assigned an ordinal denoted by $c p(a)$, e.g., $c p(0)=0$ and $c p(a)=1$ if and only if $a \in \mathcal{G}$. In case of a crossed product as described above we obtain $c p(a)=n$ for any $a=a_{1} x_{g_{1}}+\cdots+a_{n} x_{g_{n}} \in R$ if $a_{1}, \ldots, a_{n} \in F^{\times}$and if $g_{1}, \ldots, g_{n} \in G$ are pairwise distinct. For further applications it is not important to know precisely how $c p(a)$ is defined. It will be enough to know some basic rules which will enable us to apply results from [DGH] or to derive further properties. For $a, b \in D$ we write $a \unlhd b$ if $c p(a) \leq c p(b)$ and $a \triangleleft b$ if $c p(a)<c p(b)$. In the first case we say that $a$ is simpler than $b$ and in the second that $a$ is strictly simpler than $b$.

Besides the complexity of single elements we also need their formal sums. In order to explain this we first have to fix an ordinal $\Delta$ satisfying $\operatorname{cp}(a) \leq \Delta$ for all $a \in D$ and consider the free commutative monoid $\mathbb{N}(\Delta)$ freely generated by $\{\alpha \mid 0<\alpha \leq \Delta\}$. In Section 2 of [DGH] a well-ordering $\leq$ of $\mathbb{N}(\Delta)$ is defined which is compatible with the addition of $\mathbb{N}(\Delta)$. For different $x, y$ in $\mathbb{N}(\Delta)$ the relation $x<y$ can be explained as follows: There exist $\alpha_{1}, \ldots, \alpha_{k} \leq \Delta$ with $\alpha_{1}>\cdots>\alpha_{k}$ and $n_{1}, \ldots, n_{k}, m_{1}, \ldots, m_{k} \in$ $\mathbb{N} \cup\{0\}$ such that $x=n_{1} \alpha_{1}+\cdots+n_{k} \alpha_{k}$ and $y=m_{1} \alpha_{1}+\cdots+m_{k} \alpha_{k}$. Since $x \neq y$ there is a minimal $i \in\{1, \ldots, k\}$ satisfying $n_{i} \neq m_{i}$. Then $x<y$ if and only if $n_{i}<m_{i}$.

Additive Decompositions. If an element $a$ of $D$ can be written as a sum of elements of $D$ which are all strictly simpler than $a$ then $a$ is said to be additively decomposable (otherwise $a$ is called additively indecomposable). Among all these decompositions there are some which are minimal in a certain sense. These specific decompositions can be described by a function $\tau: D \longrightarrow \mathbb{N}(\Delta)$ introduced in [DGH] as follows. Let $a$ be additively decomposable and let $a_{1}, \ldots, a_{n}$ be non-zero elements of $D$ such that $a=a_{1}+\cdots+a_{n}$ and $a_{1}, \ldots, a_{n} \triangleleft a$. Then $a=a_{1}+\cdots+a_{n}$ is called a complete additive decomposition of $a$ if $c p\left(a_{1}\right)+\cdots+c p\left(a_{n}\right) \in \mathbb{N}(\Delta)$ is minimal in a certain sense with respect to the well-order $\leq$ of $\mathbb{N}(\Delta)$ introduced above. Following [DGH] we define $\tau(a):=c p\left(a_{1}\right)+\cdots+c p\left(a_{n}\right)$ whenever $a=a_{1}+\cdots+a_{n}$ is a complete 
additive decomposition. For formal reasons it is useful to define $\tau(a)$ even if $a$ is additively indecomposable. In this case we put $\tau(a)=c p(a)$ and call $a$ itself a complete additive decomposition of $a$. Thus, $a \in D$ is additively indecomposable if and only if $\tau(a)=c p(a)$.

Multiplicative Decompositions. Similar to $\tau$ there exists a function $\rho$ which describes minimal multiplicative decompositions of elements in $D$. Let $a \in D$ be additively indecomposable. Then $a$ is said to be multiplicatively decomposable if $a$ can be written as a product of elements of $D$ which are all strictly simpler than $a$. Otherwise $a$ is called an atom. For example, 0 and the elements from $\mathcal{G}$ are atoms. All other atoms are called proper. Formulations like $a$ is multiplicatively decomposable or $a$ is an atom always include that $a$ is additively indecomposable. If $a$ is multiplicatively decomposable then a decomposition $a=a_{1} \cdots a_{n}$ of $a$ such that $a_{1}, \ldots, a_{n} \triangleleft a$ is called complete if $c p\left(a_{1}\right)+\cdots+c p\left(a_{n}\right) \in \mathbb{N}(\Delta)$ is minimal in a certain sense with respect to the well-order $\leq$ of $\mathbb{N}(\Delta)$ introduced above. In this case we define $\rho(a)=c p\left(a_{1}\right)+\cdots+c p\left(a_{n}\right)$ and moreover if $a$ is an atom we put $\rho(a)=c p(a)$ and call $a$ itself a complete multiplicative decomposition of $a$. Thus, if $a$ is additively indecomposable then $a$ is an atom if and only if $\rho(a)=c p(a)$.

We return to the example where $T$ contains the subring $R=F[G, \eta, \alpha]$ with a skew field $F$, a left-ordered group $G$, and $\mathcal{G}=F^{\times} X_{G}$. Clearly, the subring $D_{[0]}$ of $T$ generated by $\mathcal{G}$ coincides with $F[G, \eta, \alpha]$. Let $a=a_{1} x_{g_{1}}+\cdots+a_{n} x_{g_{n}} \in R$ such that $a_{1}, \ldots, a_{n} \in F^{\times}$ and $g_{1}, \ldots, g_{n} \in G$ are pairwise distinct. Then, $a=a_{1} x_{g_{1}}+\cdots+a_{n} x_{g_{n}}$ is a complete additive decomposition of $a$. Moreover, $a$ is no unit in $R$ if $n>1$ by Proposition 3.3. If $n=2$ and if $a$ is a unit in $T$ then $a^{-1}=\left(a_{1} x_{g_{1}}+a_{2} x_{g_{2}}\right)^{-1}$ is a proper atom in $D$.

The next two propositions will be needed in Section 8. They are not included in [DGH] but they can easily be derived.

Proposition 4.1. Let $x \in D, c p(x)>1$ and $g \in \mathcal{G}$. If $x$ is additively indecomposable then $g x$ is also additively indecomposable and if $x$ is an atom then $g x$ is also an atom. If $x$ is additively indecomposable and $x=x_{1} \cdot x_{2} \cdots x_{n}$ is a complete multiplicative decomposition of $x$ then $c p\left(x_{i}\right)>1$ for all $i=1, \ldots, n$ and $g x=\left(g x_{1}\right) \cdot x_{2} \cdots x_{n}$ is a complete multiplicative decomposition of $g x$. If $x$ is additively decomposable with complete additive decomposition $x=x_{1}+\cdots+x_{n}$ then $g x=g x_{1}+\cdots+g x_{n}$ is a complete additive decomposition of $g x$.

Proof. Let $x$ be additively indecomposable and assume that $g x$ is additively decomposable. Then $g x$ can be written as $g x=a_{1}+a_{2}$ where $a_{1}, a_{2} \triangleleft g x$ and therefore $x=g^{-1} a_{1}+g^{-1} a_{2}$ where $c p(x)=c p(g x)$ and $c p\left(a_{i}\right)=c p\left(g^{-1} a_{i}\right)$ for $i=1,2$ by [DGH, Proposition 4.8]. Thus, $x$ is additively decomposable - a contradiction. Next, let $x$ be an atom. Again, we assume that $g x$ is not an atom. Since $g x$ is additively indecomposable there exist $a_{1}, a_{2} \in D$ such that $g x=a_{1} a_{2}$ where $a_{1}, a_{2} \triangleleft g x$. Then $x=\left(g^{-1} a_{1}\right) a_{2}$ with $g^{-1} a_{1}, a_{2} \triangleleft x$ which is a contradiction. Now, let $x$ be additively indecomposable and let $x=x_{1} \cdot x_{2} \cdots x_{n}$ be a complete multiplicative decomposition of $x$. Any $x_{i}, i=1, \ldots, n$ is an atom by [DGH, Theorem 4.6]. Without loss of generality we just show $\operatorname{cp}\left(x_{1}\right)>1$ and assume $c p\left(x_{1}\right)=1$. Then $n>1$ and $x^{\prime}=x_{1} x_{2}$ is additively indecomposable because of [DGH, Lemma 4.5]. Furthermore, $x^{\prime}=x_{1} x_{2}$ is a complete multiplicative decomposition of $x^{\prime}$ by [DGH, Theorem 4.6] such that 
$\rho\left(x^{\prime}\right)=c p\left(x_{1}\right)+c p\left(x_{2}\right)$. On the other side $x^{\prime}$ is an atom since $x_{1} \in \mathcal{G}$ by assumption. This leads to the contradiction $\rho\left(x^{\prime}\right)=c p\left(x^{\prime}\right)$. Furthermore, let $g x=a_{1} \cdot a_{2} \cdots a_{m}$ be a complete multiplicative decomposition of $g x$. According to [DGH, Proposition 4.2,4.8] and [DGH, Theorem 4.4] we obtain

$$
\begin{aligned}
\rho(x) & \leq c p\left(g^{-1} a_{1}\right)+c p\left(a_{2}\right)+\cdots+c p\left(a_{m}\right) \\
& =c p\left(a_{1}\right)+c p\left(a_{2}\right)+\cdots+c p\left(a_{m}\right)=\rho(g x) \\
& \leq c p\left(g x_{1}\right)+c p\left(x_{2}\right)+\cdots+c p\left(x_{n}\right) \\
& =c p\left(x_{1}\right)+c p\left(x_{2}\right)+\cdots+c p\left(x_{n}\right)=\rho(x)
\end{aligned}
$$

where $\rho$ is the function introduced above. Thus, $\rho(g x)=c p\left(g x_{1}\right)+c p\left(x_{2}\right)+\cdots+c p\left(x_{n}\right)$ which implies the claim because of [DGH, Proposition 4.2].

Finally, let $x$ be additively decomposable with complete additive decomposition $x=$ $x_{1}+\cdots+x_{n}$ and let $g x=a_{1}+\cdots+a_{m}$ be a complete additive decomposition of $g x$. We apply [DGH, Proposition 3.2,4.8] and [DGH, Theorem 3.4] and obtain by arguments similar to those we just used above

$$
\begin{aligned}
\tau(x) & \leq c p\left(g^{-1} a_{1}\right)+\cdots+c p\left(g^{-1} a_{m}\right)=c p\left(a_{1}\right)+\cdots+c p\left(a_{m}\right)=\tau(g x) \\
& \leq c p\left(g x_{1}\right)+\cdots+c p\left(g x_{n}\right)=c p\left(x_{1}\right)+\cdots+c p\left(x_{n}\right)=\tau(x) .
\end{aligned}
$$

Therefore, $\tau(g x)=c p\left(g x_{1}\right)+\cdots+c p\left(g x_{n}\right)$ which implies the claim because of [DGH, Proposition 3.2].

Remark. Corresponding statements hold true for terms like $x g$.

Definition 4.2. Let the notation be as above.

(1) $\Lambda=\{c p(x) \mid x \in D, x \neq 0$, and $x$ is additively indecomposable $\}$.

(2) If $\lambda \in \Lambda$ then $D_{\leq \lambda}$ denotes the subgroup of the additive group of $D$ which is generated by all $a \in D$ satisfying $c p(a) \leq \lambda$.

(3) If $\lambda \in \Lambda$ then $D_{<\lambda}$ denotes the subgroup of the additive group of $D$ which is generated by all $a \in D$ satisfying $c p(a)<\lambda$.

Proposition 4.3. With the notation as above the following hold true:

(1) For all $\lambda \in \Lambda, a \in D_{\leq \lambda}$ there are $a_{1}, \ldots, a_{n} \in D$ such that $a=a_{1}+\cdots+a_{n}$ and $c p\left(a_{1}\right), \ldots, c p\left(a_{n}\right) \leq \lambda$.

(2) $D_{<\lambda}=\{a \in D \mid c p(a)<\lambda\}$ for all $\lambda \in \Lambda$.

(3) Let $a \in D, a \neq 0$, and $\lambda \in \Lambda$. If $a=a_{1}+\cdots+a_{n}$ is a complete additive decomposition of a then a belongs to $D_{\leq \lambda}$ if and only if $c p\left(a_{1}\right), \ldots, c p\left(a_{n}\right) \leq \lambda$.

(4) If $\lambda \in \Lambda, a \in D_{\leq \lambda}$, and $b \in D$ such that $b \triangleleft a$ then $b \in D_{\leq \lambda}$.

(5) $D_{\leq \lambda} \subset D_{\leq \lambda^{\prime}}$ for all $\lambda, \lambda^{\prime} \in \Lambda$ such that $\lambda<\lambda^{\prime}$.

(6) Let $a \in D, c p(a)>1$ be additively indecomposable. For all $a_{1}, \ldots, a_{n} \in D$ such that $a_{1}, \ldots, a_{n} \triangleleft a$ there exists $\lambda \in \Lambda$ with $\lambda<c p(a)$ and $a_{1}, \ldots, a_{n} \in D_{\leq \lambda}$.

(7) For all $\lambda \in \Lambda$ and non-zero $x \in D_{<\lambda}$ there exists $\lambda^{\prime} \in \Lambda$ such that $\lambda^{\prime}<\lambda$ and $x \in D_{\leq \lambda^{\prime}}$.

Proof. (1): Clearly, $-1 \in \mathcal{G}$ since $\mathcal{G}=-\mathcal{G}$ by assumption and [DGH, Proposition 4.8] proves the claim. 
(2): This statement follows from [DGH, Proposition 3.1].

(3): Let $a$ be non-zero and let $a=a_{1}+\cdots+a_{n}$ be a complete additive decomposition of $a$. If $c p\left(a_{1}\right), \ldots, c p\left(a_{n}\right) \leq \lambda$ then $a \in D_{\leq \lambda}$ by definition of $D_{\leq \lambda}$. Conversely, let $a$ be in $D_{\leq \lambda}$, that is, $a=c_{1}+\cdots+c_{m}$ where $c_{1}, \ldots, c_{m} \in D$ and $c p\left(c_{1}\right), \ldots, c p\left(c_{m}\right) \leq \lambda$. It will be enough to show that for all $i=1, \ldots, n$ there is an index $j=1, \ldots, m$ such that $a_{i} \unlhd c_{j}$. Let us assume that there exists an $i \in\{1, \ldots, n\}$ such that $c_{j} \triangleleft a_{i}$ for all $j=1, \ldots, m$ which means $c p\left(c_{1}\right)+\cdots+c p\left(c_{m}\right)<c p\left(a_{1}\right)+\cdots+c p\left(a_{n}\right)$ with respect to the order defined in $\mathbb{N}(\Delta)$. Then $c p\left(c_{1}\right)+\cdots+c p\left(c_{m}\right)<\tau(a)$ because of [DGH, Proposition 3.2]. Applying [DGH, Lemma 3.3] we conclude $a=c_{1}+\cdots+c_{m} \triangleleft a$, a contradiction.

(4): Let $a=a_{1}+\cdots+a_{n}$ and $b=b_{1}+\cdots+b_{m}$ be two complete additive decompositions of $a$ and $b$ respectively. Then $a \neq 0$ since $b \triangleleft a$ and (3) yields $c p\left(a_{1}\right), \ldots, c p\left(a_{n}\right) \leq \lambda$. We apply [DGH, Theorem 3.4] to obtain $\tau(b) \triangleleft \tau(a)$. Thus, for any $i=1, \ldots, m$ there exists an index $j=1, \ldots, n$ such that $b_{i} \unlhd a_{j}$, that is $c p\left(b_{i}\right) \leq c p\left(a_{j}\right) \leq \lambda$. This implies $b \in D_{\leq \lambda}$.

(5): Obviously, $\lambda<\lambda^{\prime}$ provides $D_{\leq \lambda} \subseteq D_{\leq \lambda^{\prime}}$. We choose a non-zero $a \in D$ which is additively indecomposable such that $c p(a)=\lambda^{\prime}$. Then $a$ is a complete additive decomposition of $a$ and $a \in D_{\leq \lambda}$ cannot hold true because of (3).

(6): It will be enough to show that for any $a^{\prime} \in D$ satisfying $a^{\prime} \triangleleft a$ there exists $\lambda \in \Lambda$ such that $a^{\prime} \in D_{\leq \lambda}$ and $\lambda<c p(a)$. If $a^{\prime}=0$ we put $\lambda=1$. Thus, let $a^{\prime} \neq 0$. If $a^{\prime}$ is additively indecomposable we define $\lambda=c p\left(a^{\prime}\right)$. Now, let $a^{\prime}$ be additively decomposable and let $a^{\prime}=a_{1}^{\prime}+\cdots+a_{m}^{\prime}$ be a complete additive decomposition of $a^{\prime}$ where $m>1$. We assume $c p(a) \leq c p\left(a_{i}^{\prime}\right)$ for some $i \in\{1, \ldots, m\}$. Then $c p(a)<c p\left(a_{1}^{\prime}\right)+\cdots+c p\left(a_{m}^{\prime}\right)$ with respect to the given order of $\mathbb{N}(\Lambda)$ and therefore $c p\left(a_{1}^{\prime}\right)+\cdots+c p\left(a_{m}^{\prime}\right)=\tau\left(a^{\prime}\right)<$ $c p\left(a^{\prime}\right)$ by [DGH, Proposition 3.2]. This implies the contradiction $c p(a)<c p\left(a^{\prime}\right)$ and therefore $c p\left(a_{i}^{\prime}\right)<c p(a)$ for all $i=1, \ldots, m$. We may choose $\lambda$ as the maximum of all $c p\left(a_{1}^{\prime}\right), \ldots, c p\left(a_{m}^{\prime}\right)$ since any $a_{i}^{\prime}, i=1, \ldots, m$ is additively indecomposable by [DGH, Theorem 3.6].

(7): If $x$ is additively indecomposable we choose $\lambda^{\prime}=c p(x)$. Thus, let $x=a_{1}+\cdots+a_{n}$ be a complete additive decomposition of $x$ where $n>1$ and let $a$ be a non-zero element of $D$ with $\lambda=c p(a)$. Because of $a_{1}, \ldots, a_{n} \triangleleft x \triangleleft a$ and (6) there exists $\lambda^{\prime} \in \Lambda$ satisfying $\lambda^{\prime}<\lambda$ and $a_{1}, \ldots, a_{n} \in D_{\leq \lambda^{\prime}}$, that is $x \in D_{\leq \lambda^{\prime}}$.

\section{Free Division Rings of Fractions}

In this section we will use all definitions and results from Section 3 without any further explanation and fix the following notation. $R=F[G, \eta, \alpha]$ always denotes a crossed product of $G$ over $F$ with basis $X_{G}=\left\{x_{g} \mid g \in G\right\}$ where $G$ is a group with left-order $\leq$ and $F$ a skew field such that $\mathcal{G}=F^{\times} X_{G}$ is the group of units in $R$. The fact that $G$ is left-ordered is not always needed but this does not appear to be relevant here. Furthermore, $D$ is a division ring of fractions of $R$. If $U$ is a subgroup of $G$ then $F^{\times} X_{U}$ is a subgroup of $\mathcal{G}$ and $D_{U}$ denotes the rational closure of $F^{\times} X_{U}$ in $D$, that is, $D_{U}$ is a division ring of fractions of the crossed product $F[U, \eta, \alpha]$.

Let $U$ be a normal subgroup of $G$ and $h$ an element of $G$. If $t \in \mathcal{G}$ is a preimage of $h$ with 
respect to the canonical group homomorphism $\mathcal{G} \longrightarrow G, a x_{g} \longmapsto g$, that is, $t=a x_{h}$ for some $a \in F^{\times}$, then $t d t^{-1} \in D_{U}$ holds true for all $d \in D_{U}$. This can easily be shown for instance by transfinite induction on the complexity $c p(d)$ of $d$ since $U$ is normal in $G$ where here $c p(d)$ means the complexity of $d$ with respect to the fact that $D_{U}$ is the rational closure of $F[U, \eta, \alpha]$ and therefore of $F^{\times} X_{U}$ in $D$. This implies that the subring of $D$ which is generated by $D_{U}$ and $t$ is the set of all left (and right) polynomials in $t$ over $D_{U}$ which will be denoted by $D_{U}[t]$. We say that $t$ is (left) algebraic over $D_{U}$ if there exist $k \in \mathbb{N}$ and $d_{0}, d_{1}, \ldots, d_{k-1} \in D_{U}$ such that $t^{k}+d_{k-1} t^{k-1}+\cdots+d_{1} t+d_{0}=0$ where (right) algebraic is defined accordingly. Of course both properties are equivalent for $t$ and if $t$ is not algebraic we call $t$ transcendental over $D_{U}$. In this case $D_{U}[t]$ is a skew polynomial ring in the indeterminate $t$ over $D_{U}$.

Definition 5.1. Let the notation be as above. A division ring of fractions $D$ of a crossed product $R=F[G, \eta, \alpha]$ of a left-ordered group $G$ over a skew field $F$ is called Hughes-free if for any non-trivial finitely generated subgroup $H$ of $G$, any normal subgroup $N$ of $H$, and any $h \in H$ such that $H / N=\langle h N\rangle$ is an infinite cyclic group each element $t \in F^{\times} x_{h}$ is transcendental over $D_{N}$.

\section{Remarks.}

(1) The definition of (Hughes-)free division rings of fractions in the original version appeared in [H1] for the first time where $G$ is assumed to be locally indicabel.

(2) If $R=F[G, \eta, \alpha]$ is given as above then it is not clear in general which basis $X_{G}$ of the $F$-vector space $F[G, \eta, \alpha]$ is meant here. For example, in Proposition 3.3 $R$ is a crossed product with respect to $X_{G}$ and $X_{G}^{\prime}$. Moreover, in the definition above besides the group $G$ also the basis $X_{G}$ occurs which leads to some kind of ambiguity. Later on we will see that we have to be more careful in order to avoid misunderstandings and we have to state precisely which basis is used.

(3) It is easy to see that in Definition 5.1 the sentence each element $t \in F^{\times} x_{h}$ is transcendental over $D_{N}$ can be replaced by the sentence there is an element $t \in F^{\times} x_{h}$ which is transcendental over $D_{N}$.

(4) In $[\mathrm{JL}]$ it is shown that any group $\operatorname{ring} F[G]$ of a locally indicable group $G$ over a field $F$ with characteristic 0 has a Hughes-free division ring of fractions. The question whether this is true for an arbitrary crossed product $F[G, \eta, \alpha]$ of a locally indicable group $G$ is still open.

Proposition 5.2. Let $D$ be a division ring of fractions of a crossed product $F[G, \eta, \alpha]$ of a left-ordered group $G$ over a skew field $F$. Then the following statements are equivalent:

(1) D is Hughes-free.

(2) If $H$ is a subgroup of $G$ and $N$ a normal subgroup of $H$ such that $H / N=\langle h N\rangle$ is an infinite cyclic group then each $t \in F^{\times} x_{h}$ is transcendental over $D_{N}$.

(3) If $H$ is a subgroup of $G, N$ a normal subgroup of $H$ such that $H / N=\langle h N\rangle$ is an infinite cyclic group and if $h_{1}, \ldots, h_{n} \in H$ such that $h_{1} N, \ldots, h_{n} N$ are pairwise distinct then $x_{h_{1}}, \ldots, x_{h_{n}}$ are linearly independent over $D_{N}$. 
Proof. " $(1) \Rightarrow(2)$ : "We assume that there is an $a \in F^{\times}$such that $t=a x_{h}$ is algebraic over $D_{N}$, that is, there exist $k \in \mathbb{N}$ and $d_{0}, d_{1}, \ldots, d_{k-1} \in D_{N}$ satisfying

$$
t^{k}+d_{k-1} t^{k-1}+\cdots+d_{1} t+d_{0}=0 .
$$

Clearly, $d_{0}, \ldots, d_{k-1}$ are elements of the rational closure of $F[U, \eta, \alpha]$ in $D$ where $U=\left\langle g_{1}, \ldots, g_{m}\right\rangle$ is a suitable finitely generated subgroup of $N$. We consider the subgroup $H^{\prime}$ of $H$ which is generated by $g_{1}, g_{2}, \ldots, g_{m}$, and $h$. Then, $H^{\prime}$ is a finitely generated subgroup of $G$ and $N^{\prime}:=H^{\prime} \cap N$ is a normal subgroup of $H^{\prime}$ such that $g_{1}, g_{2}, \ldots, g_{m} \in N^{\prime}$ and $h \notin N^{\prime}$. Therefore, $H^{\prime} / N^{\prime}=\left\langle h N^{\prime}\right\rangle$ is an infinite cyclic group. By construction, $d_{0}, d_{1}, \ldots, d_{k-1}$ belong to the rational closure $D_{N^{\prime}}$ of $F\left[N^{\prime}, \eta, \alpha\right]$ in $D$. Since $D$ is a Hughes-free division ring of fractions of $F[G, \eta, \alpha]$ we get the desired contradiction.

$"(2) \Rightarrow(3): "$ Because of $H / N=\langle h N\rangle$ there exist $m_{1}, \ldots, m_{n} \in \mathbb{Z}$ and non-zero $a_{1}, \ldots, a_{n} \in D_{N}$ such that $x_{h_{i}}=a_{i} x_{h}^{m_{i}}$ for all $i=1, \ldots, n$. Moreover, $m_{1}, \ldots, m_{n}$ are pairwise distinct since $h_{1} N, \ldots, h_{n} N$ are pairwise distinct. Now, if $d_{1}, \ldots, d_{n} \in D_{N}$ are given with $d_{1} x_{h_{1}}+\cdots+d_{n} x_{h_{n}}=0$ then $d_{1} a_{1} x_{h}^{m_{1}}+\cdots+d_{n} a_{n} x_{h}^{m_{n}}=0$, that is $d_{1}=\cdots=d_{n}=0$ for $x_{h}$ is transcendental over $D_{N}$ and $a_{1}, \ldots, a_{n} \neq 0$.

$"(3) \Rightarrow(1)$ : " Let $H, N$, and $h$ are assumed as given in Definition 5.1 and let $d_{0}, \ldots, d_{k}$ are elements of $D_{N}$ with $d_{k} x_{h}^{k}+\cdots+d_{1} x_{h}+d_{0}=0$. Since $h N$ has infinite order in $H / N$ the cosets $h^{k} N, \ldots, h N, N$ are pairwise distinct. Thus, $x_{h}^{k}, \ldots, x_{h}, x_{e}$ are linearly independent over $D_{N}$, that is $d_{k}=\cdots=d_{0}=0$.

Proposition 5.2 shows that the two definitions of Hughes-freeness presented in [H1 and [L] for locally indicable groups coincide. It also gives rise to the following definition.

Definition 5.3. Let $D$ be a division ring of fractions of a crossed product $F[G, \eta, \alpha]$ of a left-ordered group $G$ over a skew field $F$. Then $D$ is called strongly Hughes-free if the following holds true: If $H$ is an arbitrary subgroup of $G$ with normal subgroup $N$ of $H$ and if $h_{1}, \ldots, h_{n} \in H, n \in \mathbb{N}$ are given such that $h_{1} N, \ldots, h_{n} N$ are pairwise different, then $x_{h_{1}}, \ldots, x_{h_{n}}$ are linearly independent over the rational closure $D_{N}$ of $F[N, \eta, \alpha]$ in D.

The definition of strongly Hughes-free division rings of fractions in the original version appeared in $[\mathrm{L}]$ where $G$ is assumed to be locally indicabel. The following proposition holds true more generally but in Section 9 we just need this version.

Proposition 5.4. Let the notation be as in Proposition 3.3 and let $D$ be a (strongly) Hughes-free division ring of fractions of $R=F[G, \eta, \alpha]$ with respect to $X_{G}$. Then $D$ is also (strongly) Hughes-free with respect to $X_{G}^{\prime}$.

Proof. We just consider the case where $D$ is strongly Hughes-free. For any $g \in G$ there are uniquely determined $a_{g} \in F^{\times}$and $g^{\prime} \in G$ such that $x_{g}^{\prime}=\varphi\left(x_{g}\right)=a_{g} x_{g^{\prime}}$. The mapping $\psi: G \longrightarrow G, g \longmapsto g^{\prime}$ is bijective and for all $g, h \in G$ we get $\varphi\left(x_{g h}\right)=a_{g h} x_{\psi(g h)}$ and $\varphi\left(x_{g h}\right)=\varphi\left(\eta(g, h)^{-1}\right) a_{g} \alpha_{\psi(g)}\left(a_{h}\right) \eta(\psi(g), \psi(h)) x_{\psi(g) \psi(h)}$. Thus, $\psi$ is a group automorphism. In order to simplify the notation let an apostrophe indicate the image of a term under $\psi$. Now, let $H, N$, and $h_{1}, \ldots, h_{n} \in H$ are given as in Definition 5.3. Then $H^{\prime}$ is a subgroup of $G, N^{\prime}$ a normal subgroup of $H^{\prime}$, and $h_{1}^{\prime} N^{\prime}, \ldots, h_{n}^{\prime} N^{\prime}$ are pairwise 
distinct. Clearly, $F^{\times} X_{N}^{\prime}$ and $F^{\times} X_{N^{\prime}}$ generate the same subring of $D$ such that $D_{N^{\prime}}$ is also the rational closure of $F^{\times} X_{N}^{\prime}$ in $D$. By assumption, $a_{h_{1}} x_{h_{1}^{\prime}}, \ldots, a_{h_{n}} x_{h_{n}^{\prime}}$ are linearly independent over $D_{N^{\prime}}$ where $a_{h_{i}} x_{h_{i}^{\prime}}=x_{h_{i}}^{\prime}$ for all $i=1, \ldots, n$.

Next we introduce another notion of freeness in case that $\leq$ is a Conradian left-order of $G$. For any convex jump $\left(C^{\prime}, C\right)$ of $G$ with respect to $\leq$ let $D_{C}^{+}$be the rational closure of $F[C, \eta, \alpha]$ and $D_{C}^{-}$the rational closure of $F\left[C^{\prime}, \eta, \alpha\right]$ in $D$. If $g \in C \backslash C^{\prime}$, that is $C=C_{g}^{+}$and $C^{\prime}=C_{g}^{-}$, we will also write $D_{g}^{+}$and $D_{g}^{-}$instead of $D_{C}^{+}$and $D_{C}^{-}$ respectively. Then $t d t^{-1} \in D_{g}^{-}$for all $d \in D_{g}^{-}$and $t \in F^{\times} x_{g}$ since $C_{g}^{-}$is normal in $C_{g}^{+}$.

Definition 5.5. Let $D$ be a division ring of fractions of the crossed product $F[G, \eta, \alpha]$ of a group $G$ with Conradian left-order $\leq$ over a skew field $F$. Then $D$ is called free with respect to $\leq$ if for any convex jump $\left(C^{\prime}, C\right)$ of $G$ with respect to $\leq$ the following holds true: If $h_{1}, \ldots, h_{n} \in C, n \in \mathbb{N}$ are given such that $h_{1} C^{\prime}, \ldots, h_{n} C^{\prime}$ are pairwise distinct, then $x_{h_{1}}, \ldots, x_{h_{n}}$ are linearly independent over $D_{C}^{-}$.

Proposition 5.6. Let $D$ be a division ring of fractions of the crossed product $R=$ $F[G, \eta, \alpha]$ where $G$ is a locally indicable group and $F$ is a skew field. If $D$ is strongly Hughes-free then $D$ is free with respect to any Conradian left-order $\leq$ of $G$ and if $D$ is Hughes-free then $D$ is free with respect to any Conradian left-order $\leq$ of $G$ with maximal rank.

Proof. If $D$ is strongly Hughes-free then the statement is obvious. Thus, let $\leq$ be a Conradian left-order of $G$ with maximal rank, let $\left(C^{\prime}, C\right)$ be a convex jump of $G$ with respect to $\leq$, and let $h_{1}, \ldots, h_{n} \in C, n \in \mathbb{N}$ are given such that $h_{1} C^{\prime}, \ldots, h_{n} C^{\prime}$ are pairwise different. We define $H=H^{\prime} C^{\prime}$ where $H^{\prime}$ is the subgroup of $C$ generated by $h_{1}, \ldots, h_{n}$. Then $C^{\prime}$ is a normal subgroup of $H$ and $H / C^{\prime}$ is the subgroup of $C / C^{\prime}$ generated by $h_{1} C^{\prime}, \ldots, h_{n} C^{\prime}$. Hence, $H / C^{\prime}$ is cyclic since $C / C^{\prime}$ is isomorphic to a subgroup of the additive group $\mathbb{Q}$. If $H=C^{\prime}$ then $h_{1}, \ldots, h_{n} \in C^{\prime}$, that is, $n=1$ and we are done. Otherwise, Proposition $\$ 5.2$ can be applied.

The second part of Proposition 5.6 also appears in [J].

\section{A Structure Theorem for Free Division Rings of Fractions}

Throughout this section we shall fix the following notation. $G$ is a (locally indicable) group with Conradian left-order $\leq$ and $F[G, \eta, \alpha]$ is a crossed product of $G$ over a skew field $F$ having a division ring of fractions $D$ which is free with respect to $\leq$. The convex subgroups of $G$ with lower neighbour in $\mathcal{C}_{\leq}$shall be called convex successors (of $G$ with respect to $\leq$ ). They form a subset $\mathcal{C}_{\leq}^{*}$ of $\mathcal{C}_{\leq}$and if $C \in \mathcal{C}_{\leq}^{*}$ is given then $C^{\prime}$ always denotes the lower neighbour of $C$ such that $\left(C^{\prime}, C\right)$ is a convex jump. For example, $C_{g}^{+}$is a convex successor whenever $g \neq e$. We intend to continue using the notation $D_{C}^{+}, D_{C}^{-}, D_{g}^{+}$, and $D_{g}^{-}$as introduced before Definition 5.5 . For any $C \in \mathcal{C}_{\leq}^{*}$ we also fix a transversal $\mathfrak{C}$ for $C^{\prime}$ in $C$ satisfying $e \in \mathfrak{C}$ and $\overline{\mathfrak{C}}, \tilde{\mathfrak{C}}$ are defined analogously for 
$\bar{C}, \tilde{C} \in \mathcal{C}_{\leq}^{*}$. According to Proposition 3.1 the ring $F[C, \eta, \alpha]$ now turns into a crossed product of $C / C^{\prime}$ over $F\left[C^{\prime}, \eta, \alpha\right]$ with respect to the basis $X_{C / C^{\prime}}=\left\{x_{g} \mid g \in \mathfrak{C}\right\}$. Since $D$ is free with respect to $\leq$ the elements of $X_{C / C^{\prime}}$ are linearly independent over $D_{C}^{-}$. This means that the subring of $D$ generated by $D_{C}^{-}$and $X_{C / C^{\prime}}$ is a crossed product of $C / C^{\prime}$ over $D_{C}^{-}$with respect to the basis $X_{C / C^{\prime}}$ which will be denoted by $D_{C}^{-}\left[C / C^{\prime}, \eta, \alpha\right]$. Clearly, $D_{C}^{+}$is the rational closure of $D_{C}^{-}\left[C / C^{\prime}, \eta, \alpha\right]$ in $D$. Because of Proposition 2.9 and Proposition 3.4 the ring $D_{C}^{-}\left[C / C^{\prime}, \eta, \alpha\right]$ is an Ore domain for $C / C^{\prime}$ is abelian and therefore $D_{C}^{+}$is the corresponding Ore division ring of fractions. Next we consider $D_{C}^{-}\left[C / C^{\prime}, \eta, \alpha\right]$ as a subring of the skew field $D_{C}^{-}\left(\left(C / C^{\prime}, \eta, \alpha\right)\right)$ of all formal power series in $C / C^{\prime}$ over $D_{C}^{-}$as given in $\left[\mathrm{Ne}\right.$ that also contains $D_{C}^{+}$as a subdivision ring in a unique manner because $D_{C}^{-}\left[C / C^{\prime}, \eta, \alpha\right]$ meets the Ore condition. Hence, any element $x \in D_{C}^{+}$possesses a unique representation $x=\sum_{g \in \mathfrak{C}} d_{g} x_{g}$ as a formal power series with $d_{g} \in D_{C}^{-}$for all $g \in \mathfrak{C}$ such that the support $\left\{g \in \mathfrak{C} \mid d_{g} \neq 0\right\}$ is well-ordered with respect to the given Conradian left-order of $G$.

Unless otherwise specified, the complexity $c p(d)$ of an element $d \in D$ always refers to the way $d$ is built from elements of $\mathcal{G}=F^{\times} X_{G}$.

Theorem 6.1. With the assumptions stated above, for any $x \in D$ with $c p(x)>1$ the following hold true:

(1) There exist $h \in G$ and $C \in C_{\leq}^{*}$ such that $x_{h}^{-1} x \in D_{C}^{+}$and $x_{h}^{-1} x=\sum_{g \in \mathfrak{C}} d_{g} x_{g}$ where $d_{g} \in D_{C}^{-}$with $d_{g} \triangleleft x$ for all $g \in \mathfrak{C}$.

(2) There exist $h \in G$ and $C \in C_{\leq}^{*}$ such that $x x_{h}^{-1} \in D_{C}^{+}$and $x x_{h}^{-1}=\sum_{g \in \mathfrak{C}} d_{g} x_{g}$ where $d_{g} \in D_{C}^{-}$with $d_{g} \triangleleft x$ for all $g \in \mathfrak{C}$.

\section{Remarks.}

(1) We call $x_{h}^{-1} x=\sum_{g \in \mathfrak{C}} d_{g} x_{g}$ as given above a left representation of $x$ and then $x_{\bar{h} h}^{-1}\left(x_{\bar{h}} x\right)=\sum_{g \in \mathfrak{C}}\left(\alpha_{\bar{h} h}^{-1}(\eta(\bar{h}, h)) d_{g}\right) x_{g}$ is a left representation of $x_{\bar{h}} x$ for all $\bar{h} \in G$. Similarly, $x x_{h}^{-1}=\sum_{g \in \mathfrak{C}} d_{g} x_{g}$ as given above is called a right representation of $x$ and $\left(x x_{\bar{h}}\right) x_{h \bar{h}}^{-1}=\sum_{g \in \mathfrak{C}}\left(d_{g} \alpha_{g}(\eta(h, \bar{h}))\right) x_{g}$ is a right representation of $x x_{\bar{h}}$ for all $\bar{h} \in G$.

(2) We will see later in Theorem 6.2 that the left and right representations of an element $x \in D, c p(x)>1$ is unique in a certain sense.

(3) Because of $d_{g} \triangleleft x$ for all $g \in \mathfrak{C}$ there are at least two non-zero summands in any left and right representation of $x$.

Proof of Theorem 6.1. We will prove both statements of the theorem simultaniously by transfinite induction on the complexity of $x$ and consider the case $x \in F[G, \eta, \alpha]$ first. Then

$$
x=a_{1} x_{g_{1}}+a_{2} x_{g_{2}}+\cdots+a_{n} x_{g_{n}}
$$

where $a_{1}, \ldots, a_{n} \in F^{\times}$and $g_{1}, \ldots, g_{n} \in G$ are pairwise distinct. Moreover, $n \geq 2$ because of $c p(x)>1$. We may choose notation so that $C_{g_{1}}^{+} \subseteq \cdots \subseteq C_{g_{n}}^{+}$and two different cases occur.

Case 1: $C_{g_{1}}^{+} \subset C_{g_{n}}^{+}$. Then

$$
C_{g_{1}}^{+} \subseteq \cdots \subseteq C_{g_{m}}^{+} \subset C_{g_{m+1}}^{+}=\cdots=C_{g_{n}}^{+}
$$


for some $m<n$. We put $C:=C_{g_{n}}^{+}$, that is $C^{\prime}:=C_{g_{n}}^{-}$, and $d_{e}=a_{1} x_{g_{1}}+\cdots+a_{m} x_{g_{m}} \in D_{C}^{-}$ with $d_{e} \triangleleft x$ since $m<n$. Collecting all summands in $a_{m+1} x_{g_{m+1}}+\cdots+a_{n} x_{g_{n}}$ that correspond to the same cosets of $C^{\prime}$ there exist pairwise distinct $h_{1}, \ldots, h_{r} \in \mathfrak{C}$ such that $a_{m+1} x_{g_{m+1}}+\cdots+a_{n} x_{g_{n}}=d_{h_{1}} x_{h_{1}}+\cdots+d_{h_{r}} x_{h_{r}}$ where $d_{h_{i}} \in D_{C}^{-}$and $d_{h_{i}} \triangleleft x$ for all $i=1, \ldots, r$. Finally, we put $h=e$ for both parts of the theorem and obtain

$$
x_{h}^{-1} x=d_{e} x_{e}+d_{h_{1}} x_{h_{1}}+\cdots+d_{h_{r}} x_{h_{r}}=x x_{h}^{-1}
$$

as desired.

Case 2: $C_{g_{1}}^{+}=C_{g_{2}}^{+}=\cdots=C_{g_{n}}^{+}$. Because of $n \geq 2$ there exists at least one index $i$ such that $g_{i}$ is not trivial which yields that $g_{i}$ is not trivial for all $i=1,2, \ldots, n$. Defining $h=g_{1}$ provides

$$
\begin{gathered}
x_{h}^{-1} x=a_{1}^{\prime} x_{e}+a_{2}^{\prime} x_{g_{1}^{-1} g_{2}}+\cdots+a_{n}^{\prime} x_{g_{1}^{-1} g_{n}}, \\
x x_{h}^{-1}=\tilde{a}_{1} x_{e}+\tilde{a}_{2} x_{g_{2} g_{1}^{-1}}+\cdots+\tilde{a}_{n} x_{g_{n} g_{1}^{-1}}
\end{gathered}
$$

where $g_{1}^{-1} g_{i}, g_{i} g_{1}^{-1} \neq e$ for $i \geq 2$ and $a_{1}^{\prime}, \ldots, a_{n}^{\prime}, \tilde{a}_{1}, \ldots, \tilde{a}_{n} \in F^{\times}$. We can now proceed analogously to Case 1 again.

We next turn to the case $x \notin D_{[0]}$ and assume that the theorem has been proved for all elements in $D$ which are strictly simpler than $x$. First of all, let $x$ be an atom. Then $x^{-1} \triangleleft x$ by [DGH, Proposition 4.1]. There exist $h \in G$ and $C \in \mathcal{C}_{\leq}^{*}$ such that $x_{h}^{-1} x^{-1} \in D_{C}^{+}$and

$$
x_{h}^{-1} x^{-1}=\sum_{g \in \mathfrak{C}} d_{g} x_{g} \text { where } d_{g} \in D_{C}^{-} \text {with } d_{g} \triangleleft x^{-1} \text { for all } g \in \mathfrak{C} .
$$

From this we will deduce a right representation $x x_{h}=\sum_{\bar{g} \in \mathfrak{C}} \bar{d}_{\bar{g}} x_{\bar{g}} \in D_{C}^{+}$of $x x_{h}$ where $\bar{d}_{\bar{g}} \in D_{C}^{-}$and $\bar{d}_{\bar{g}} \triangleleft x$ for all $\bar{g} \in \mathfrak{C}$ such that $x x_{h^{-1}}^{-1}=\sum_{\bar{g} \in \mathfrak{C}}\left(\bar{d}_{\bar{g}} \alpha_{\bar{g}}\left(\eta\left(h^{-1}, h\right)^{-1}\right)\right) x_{\bar{g}}$ is a right representation of $x$ since $\bar{d}_{\bar{g}} \alpha \bar{g}\left(\eta\left(h^{-1}, h\right)^{-1}\right) \triangleleft x$ by [DGH, Proposition 4.8].

Now, let $g_{0}$ be minimal in the support of $x_{h}^{-1} x^{-1}$. For any $g$ from this support, $g>g_{0}$ there exist uniquely determined $g^{\prime} \in \mathfrak{C}$ and $a_{g} \in F^{\times} X_{C^{\prime}}$ satisfying $g g_{0}^{-1} C^{\prime}=g^{\prime} C^{\prime}$ and $x_{g} x_{g_{0}}^{-1}=a_{g} x_{g^{\prime}}$. Furthermore, $g^{\prime} C^{\prime}=g_{0}^{-1} g C^{\prime}$ and $g_{0}^{-1} g>e$ for $C / C^{\prime}$ is abelian, that is $g^{\prime}>e$ and $\left\{g^{\prime} \mid d_{g} \neq 0\right\}$ is well-ordered with respect to the given order $\leq$ of $G$. Therefore,

$$
1+\sum_{g^{\prime}>e} d_{g_{0}}^{-1} d_{g^{\prime}}^{\prime} x_{g^{\prime}} \in D_{C}^{-}\left(\left(C / C^{\prime}, \eta, \alpha\right)\right)
$$

where $d_{g^{\prime}}^{\prime}:=d_{g} a_{g}$ and

$$
x_{h}^{-1} x^{-1}=d_{g_{0}}\left(1+\sum_{g^{\prime}>e} d_{g_{0}}^{-1} d_{g^{\prime}}^{\prime} x_{g^{\prime}}\right) x_{g_{0}}
$$

such that

$$
x x_{h}=x_{g_{0}}^{-1}\left(1+\sum_{g^{\prime}>e} d_{g_{0}}^{-1} d_{g^{\prime}}^{\prime} x_{g^{\prime}}\right)^{-1} d_{g_{0}}^{-1} .
$$

According to [Ne, Section4] there exists a power series representation

$$
\left(1+\sum_{g^{\prime}>e} d_{g_{0}}^{-1} d_{g^{\prime}}^{\prime} x_{g^{\prime}}\right)^{-1}=1+\sum_{\tilde{g}>e} \tilde{d}_{\tilde{g}} x_{\tilde{g}},
$$


where $\tilde{d}_{\tilde{g}} \in D_{C}^{-}$for any $\tilde{g}>e$ and next we verify $\tilde{d}_{\tilde{g}} \triangleleft x$. The proof of [Ne, Theorem 4.9] shows that any $\tilde{d}_{\tilde{g}}$ is a sum of products with factors from

$$
\Lambda=\left\{ \pm 1, d_{g_{0}}^{-1}, d_{g^{\prime}}^{\prime}, x_{g^{\prime}}, x_{g^{\prime}}^{-1} \mid g^{\prime} \in \mathfrak{C}\right\} \cup F^{\times} X_{C^{\prime}}
$$

Clearly, \pm 1 , all $x_{g^{\prime}}, x_{g^{\prime}}^{-1}$, and all elements from $F^{\times} X_{C^{\prime}}$ are strictly simpler than $x$. Moreover, [DGH, Proposition 4.1] and $d_{g} \triangleleft x^{-1}$ imply $d_{g}, d_{g}^{-1} \triangleleft x$ such that $d_{g_{0}}^{-1} \triangleleft x$ and $d_{g^{\prime}}^{\prime}=d_{g} a_{g} \triangleleft x$ by [DGH, Proposition 4.8]. Because of [DGH, Proposition 4.1] any product of elements from $\Lambda$ is strictly simpler than $x$ which yields $\tilde{d}_{\tilde{g}} \triangleleft x$. This means

$$
x x_{h}=x_{g_{0}}^{-1}\left(1+\sum_{\tilde{g}>e} \tilde{d}_{\tilde{g}} x_{\tilde{g}}\right) d_{g_{0}}^{-1}=x_{g_{0}}^{-1} d_{g_{0}}^{-1}+\sum_{\tilde{g}>e} x_{g_{0}}^{-1} \tilde{d}_{\tilde{g}} x_{\tilde{g}} d_{g_{0}}^{-1} .
$$

This presentation of $x x_{h}$ can now easily be transformed into a formal power series of the desired form since $x_{g_{0}}^{-1} d_{g_{0}}^{-1} x_{g_{0}}$ and $x_{g_{0}}^{-1} \tilde{d}_{\tilde{g}} x_{\tilde{g}} d_{g_{0}}^{-1} x_{\tilde{g}}^{-1} x_{g_{0}}$ are in $D_{C}^{-}$for all $\tilde{g}>e$ and they are all strictly simpler than $x$. Similar arguments show that $x$ also possesses a left representation if $x$ is an atom.

We continue with the case that $x$ is additively indecomposable and consider the complete multiplicative decomposition $x=x_{1} \cdot x_{2} \cdots x_{n}$ of $x$ with $n \in \mathbb{N}$. According to [DGH, Theorem 4.6] any $x_{i}, i=1, \ldots, n$ is a proper atom. We prove the claim of the theorem for $x$ by induction on $n$ using that the theorem holds true for all elements in $D$ which are strictly simpler than $x$. If $n=1$ then $x$ is a proper atom which has been treated above. Thus, let $n>1$. We define $\bar{x}:=x_{2} \cdots x_{n}$ which is additively indecomposable by [DGH, Lemma 4.5] and $\bar{x}=x_{2} \cdots x_{n}$ is a complete multiplicative decomposition of $\bar{x}$ by [DGH, Theorem 4.6]. We apply the induction hypothesis to obtain $\bar{h} \in G, \bar{C} \in \mathcal{C}_{\leq}^{*}$ such that $x_{\bar{h}}^{-1} \bar{x} \in D_{\bar{C}}^{+}$and

$$
x_{\bar{h}}^{-1} \bar{x}=\sum_{\bar{g} \in \overline{\mathfrak{C}}} \bar{d}_{\bar{g}} x_{\bar{g}} \text { with } \bar{d}_{\bar{g}} \in D_{\bar{C}}^{-} \text {and } \bar{d}_{\bar{g}} \triangleleft \bar{x} \text { for all } \bar{g} \in \overline{\mathfrak{C}} \text {. }
$$

Since $x_{1} x_{\bar{h}}$ is a proper atom there exist $\tilde{h} \in G, \tilde{C} \in \mathcal{C}_{\leq}^{*}$ such that $x_{\tilde{h}}^{-1}\left(x_{1} x_{\bar{h}}\right) \in D_{\tilde{C}}^{+}$and

$$
x_{\tilde{h}}^{-1}\left(x_{1} x_{\bar{h}}\right)=\sum_{\tilde{g} \in \tilde{\mathfrak{C}}} \tilde{d}_{\tilde{g}} x_{\tilde{g}} \text { with } \tilde{d}_{\tilde{g}} \in D_{\tilde{C}}^{-} \text {and } \tilde{d}_{\tilde{g}} \triangleleft x_{1} x_{\bar{h}} \text { for all } \tilde{g} \in \tilde{\mathfrak{C}} \text {. }
$$

We shall distinguish three cases.

Case 1: $\tilde{C} \subset \bar{C}$, that is $\tilde{C} \subseteq \bar{C}^{\prime}$, and $D_{\tilde{C}}^{+}$is a subdivision ring of $D_{\bar{C}}^{-}$. Further,

$$
x_{\tilde{h}}^{-1} x=x_{\tilde{h}}^{-1} x_{1} \bar{x}=x_{\tilde{h}}^{-1} x_{1} x_{\bar{h}} x_{\bar{h}}^{-1} \bar{x}=\sum_{\bar{g} \in \overline{\mathfrak{C}}}\left(x_{\tilde{h}}^{-1} x_{1} x_{\bar{h}} \bar{d}_{\bar{g}}\right) x_{\bar{g}} \text { with } x_{\tilde{h}}^{-1} x_{1} x_{\bar{h}} \bar{d}_{\bar{g}} \in D_{\bar{C}}^{-} .
$$

Since $x_{\tilde{h}}^{-1} x_{1} x_{\bar{h}}$ and $x_{1}$ have the same complexity we conclude $x_{\tilde{h}}^{-1} x_{1} x_{\bar{h}} \bar{d}_{\bar{g}} \triangleleft x_{1} \bar{x}=x$ because of $\bar{d}_{\bar{g}} \triangleleft \bar{x}$ and [DGH, Theorem 4.6].

Case 2: $\bar{C} \subset \tilde{C}$, that is $\bar{C} \subseteq \tilde{C}^{\prime}$, and $D_{\bar{C}}^{+}$is a subdivision ring of $D_{\tilde{C}}^{-}$. From $x_{\bar{h}}^{-1} \bar{x} \in D_{\tilde{C}}^{-}$ we get $x_{\tilde{g}} x_{\bar{h}}^{-1} \bar{x} x_{\tilde{g}}^{-1} \in D_{\tilde{C}}^{-}$and $\tilde{d}_{\tilde{g}} x_{\tilde{g}} x_{\bar{h}}^{-1} \bar{x} x_{\tilde{g}}^{-1} \in D_{\tilde{C}}^{-}$for all $\tilde{g} \in \tilde{\mathfrak{C}}^{-}$where, in addition $c p\left(x_{\tilde{g}} x_{\bar{h}}^{-1} \bar{x} x_{\tilde{g}}^{-1}\right)=c p(\bar{x})$ and $c p\left(\tilde{d}_{\tilde{g}}\right)<c p\left(x_{1} x_{\bar{h}}\right)=c p\left(x_{1}\right)$ imply $\tilde{d}_{\tilde{g}} x_{\tilde{g}} x_{\bar{h}}^{-1} \bar{x} x_{\tilde{g}}^{-1} \triangleleft x_{1} \bar{x}=x$ due to [DGH, Theorem 4.6]. Thus,

$$
x_{\tilde{h}}^{-1} x=x_{\tilde{h}}^{-1} x_{1} \bar{x}=x_{\tilde{h}}^{-1} x_{1} x_{\bar{h}} x_{\bar{h}}^{-1} \bar{x}=\sum_{\tilde{g} \in \tilde{C}}\left(\tilde{d}_{\tilde{g}} x_{\tilde{g}} x_{\bar{h}}^{-1} \bar{x} x_{\tilde{g}}^{-1}\right) x_{\tilde{g}}
$$

is a left representation of $x$. 
Case 3: $\bar{C}=\tilde{C}$. We define $C:=\bar{C}=\tilde{C}$ and obtain $x_{\bar{h}}^{-1} \bar{x}, x_{\tilde{h}}^{-1}\left(x_{1} x_{\bar{h}}\right) \in D_{C}^{+}$with left representations $x_{\bar{h}}^{-1} \bar{x}=\sum_{g \in \mathfrak{C}} \bar{d}_{g} x_{g}$ and $x_{\tilde{h}}^{-1}\left(x_{1} x_{\bar{h}}\right)=\sum_{g \in \mathfrak{C}} \tilde{d}_{g} x_{g}$ where $\bar{d}_{g}, \tilde{d}_{g} \in D_{C}^{-}$ such that $\bar{d}_{g} \triangleleft \bar{x}, \tilde{d}_{g} \triangleleft x_{1} x_{\bar{h}}$. In the skew field $D_{C}^{-}\left(\left(C / C^{\prime}, \eta, \alpha\right)\right)$ of all formal power series in $C / C^{\prime}$ over $D_{C}^{-}$we obtain

$$
x_{\tilde{h}}^{-1} x=x_{\tilde{h}}^{-1} x_{1} x_{\bar{h}} x_{\bar{h}}^{-1} \bar{x}=\left(\sum_{g \in \mathfrak{C}} \tilde{d}_{g} x_{g}\right)\left(\sum_{g \in \mathfrak{C}} \bar{d}_{g} x_{g}\right)
$$

as a product of two formal power series. The resulting series has coefficients which are sums of products with two factors each where the first factor always is simpler than $x_{1}$ and the second is simpler than $\bar{x}$. According to [DGH, Theorem 4.6] all these products are simpler than $x_{1} \bar{x}=x$. Since $x$ is additively indecomposable any sum of these products is simpler than $x$ by [DGH, Proposition 3.1]. This provides the desired left representation of $x=x_{1} \bar{x}$. Similar arguments can be used to show that $x$ has a right representation as stated in the theorem.

We finally turn to the general case where $x$ possesses a complete additive decomposition $x=x_{1}+x_{2}+\cdots+x_{n}$ and we prove the claim of the theorem for $x$ by induction on $n$. If $n=1$ then $x$ is additively indecomposable which has been treated above. Thus, let $n>1$. [DGH, Theorem 3.6] ensures that each $x_{i}$ is additively indecomposable and $\bar{x}:=x_{2}+\cdots+x_{n}$ is a complete additive decomposition of $\bar{x}$ by [DGH, Theorem 3.6]. We will admit for a moment that $c p\left(x_{1}\right), c p(\bar{x})>1$ which enable us to apply the induction hypothesis. Hence, there exist $\bar{h} \in G, \bar{C} \in \mathcal{C}_{\leq}^{*}$ such that $x_{\bar{h}}^{-1} \bar{x} \in D_{\bar{C}}^{+}$and

$$
x_{\bar{h}}^{-1} \bar{x}=\sum_{\bar{g} \in \overline{\mathfrak{C}}} \bar{d}_{\bar{g}} x_{\bar{g}} \text { with } \bar{d}_{\bar{g}} \in D_{\bar{C}}^{-} \text {and } \bar{d}_{\bar{g}} \triangleleft \bar{x} \text { for all } \bar{g} \in \overline{\mathfrak{C}} .
$$

In addition, there are $\tilde{h} \in G, \tilde{C} \in \mathcal{C}_{\leq}^{*}$ satisfying $x_{\tilde{h}}^{-1} x_{1} \in D_{\tilde{C}}^{+}$and

$$
x_{\tilde{h}}^{-1} x_{1}=\sum_{\tilde{g} \in \tilde{\mathfrak{C}}} \tilde{d}_{\tilde{g}} x_{\tilde{g}} \text { with } \tilde{d}_{\tilde{g}} \in D_{\tilde{C}}^{-} \text {and } \tilde{d}_{\tilde{g}} \triangleleft x_{1} \text { for all } \tilde{g} \in \tilde{\mathfrak{C}} \text {. }
$$

We just investigate the case $\tilde{C} \subseteq \bar{C}$. If $\bar{C} \subseteq \tilde{C}$ we proceed similarly. Again, several subcases have to be distinguished.

Case 1: $\tilde{C}=\bar{C}$. We put $h:=\bar{h}^{-1} \tilde{h}$ and consider two subcases where the first one refers to $h \in \tilde{C}=\bar{C}$. Then, we define $C:=\tilde{C}=\bar{C}$ and write

$$
x_{\bar{h}}^{-1} \bar{x}=\sum_{g \in \mathfrak{C}} \bar{d}_{g} x_{g}, x_{\tilde{h}}^{-1} x_{1}=\sum_{g \in \mathfrak{C}} \tilde{d}_{g} x_{g} \text { with } \bar{d}_{g}, \tilde{d}_{g} \in D_{C}^{-} \text {and } \bar{d}_{g} \triangleleft \bar{x}, \tilde{d}_{g} \triangleleft x_{1} .
$$

For all $g \in \mathfrak{C}$ there exist uniquely determined $g^{\prime} \in \mathfrak{C}$ and $u_{g} \in F^{\times} X_{C^{\prime}}$ such that $x_{h} x_{g}=u_{g} x_{g^{\prime}}$ and $\left\{g^{\prime} \mid \tilde{d}_{g} \neq 0\right\}$ is well-ordered with respect to $\leq$. We now introduce $\tilde{d}_{g^{\prime}}^{\prime}:=\left(x_{\bar{h}}^{-1}\left(\eta(\bar{h}, h)^{-1}\right) x_{\bar{h}}\right)\left(x_{h} \tilde{d}_{g} x_{h}^{-1}\right) u_{g} \in D_{C}^{-}$for all $g \in \mathfrak{C}$ where $\tilde{d}_{g}$ and $\tilde{d}_{g^{\prime}}^{\prime}$ have the same complexity. A straightforward calculation shows

$$
x_{\bar{h}}^{-1} x_{1}=\sum_{g^{\prime} \in \mathfrak{C}} \tilde{d}_{g^{\prime}}^{\prime} x_{g^{\prime}} \text { with } \tilde{d}_{g^{\prime}}^{\prime} \in D_{C}^{-} \text {and } \tilde{d}_{g^{\prime}}^{\prime} \triangleleft x_{1} .
$$

Therefore, in this subcase we may assume $\tilde{h}=\bar{h}$. Adding the power series representations of $x_{\bar{h}}^{-1} \bar{x}$ and $x_{\bar{h}}^{-1} x_{1}$ yields a representation of $x_{\bar{h}}^{-1}\left(x_{1}+\bar{x}\right)$ as a power series 
$\sum_{g \in \mathfrak{C}} d_{g} x_{g}$ with coefficients $d_{g}=\tilde{d}_{g}+\bar{d}_{g} \in D_{C}^{-}$where $\tilde{d}_{g} \triangleleft x_{1}$ and $\bar{d}_{g} \triangleleft \bar{x}$, that is, $d_{g} \triangleleft x$ because of [DGH, Theorem 3.6].

Now, we turn to the second subcase of $\tilde{C}=\bar{C}$ that refers to $h \notin \bar{C}$ and define $C=C_{h}^{+} \in \mathcal{C}_{\leq}^{*}$. This provides $\tilde{C}, \bar{C} \subseteq C^{\prime}$ which means that $D_{\tilde{C}}^{+}$and $D_{\bar{C}}^{+}$are subdivision rings of $D_{C}^{-}$. Thus, $x_{\bar{h}}^{-1} \bar{x}, x_{\tilde{h}}^{-1} x_{1} \in D_{C}^{-}$follows. Since $h \in C \backslash C^{\prime}$ there exist $g \in \mathfrak{C}, g \neq e$ and $u \in F^{\times} X_{C^{\prime}}$ satisfying $x_{h}^{-1}=u x_{g}$ such that

$$
x_{\tilde{h}}^{-1} x=x_{\tilde{h}}^{-1} x_{1}+x_{\tilde{h}}^{-1} \bar{x}=d_{e} x_{e}+d_{g} x_{g}
$$

with $d_{e}=x_{\tilde{h}}^{-1} x_{1}$ and $d_{g}=\left(x_{h}^{-1}\left(\left(x_{\bar{h}}^{-1} \eta(\bar{h}, h) x_{\bar{h}}\right)\left(x_{\bar{h}}^{-1} \bar{x}\right)\right) x_{h}\right) u$. Because of $d_{e}, d_{g} \in D_{C}^{-}$, $c p\left(d_{e}\right)=c p\left(x_{1}\right)<c p(x)$, and $c p\left(d_{g}\right)=c p(\bar{g})<x$ this is a left representation of $x$.

Case 2: $\tilde{C} \subset \bar{C}$. Again, let $h=\bar{h}^{-1} \tilde{h}$. As above we investigate the first subcase where $h \in \bar{C}$ and choose $C:=\bar{C}$, that is, $\tilde{C} \subseteq C^{\prime}$. Then, $x_{\tilde{h}}^{-1} x_{1} \in D_{\tilde{C}}^{+} \subseteq D_{C}^{-}$. Because of $h \in C$ there exist $g^{\prime} \in \mathfrak{C}$ and $u \in F^{\times} X_{C^{\prime}}$ such that $x_{h}=u x_{g^{\prime}}$. Now, we define $d_{g^{\prime}}^{\prime}=\left(\left(x_{\bar{h}}^{-1} \eta(\bar{h}, h)^{-1} x_{\bar{h}}\right)\left(x_{h}\left(x_{\tilde{h}}^{-1} x_{1}\right) x_{h}^{-1}\right)\right) u$ and conclude $d_{g^{\prime}}^{\prime} \in D_{C}^{-}$where in addition $c p\left(d_{g^{\prime}}^{\prime}\right)=c p\left(x_{1}\right)$. A straightforward calculation shows $x_{\bar{h}}^{-1} x_{1}=d_{g^{\prime}}^{\prime} x_{g^{\prime}}$ such that

$$
x_{\bar{h}}^{-1} x=x_{\bar{h}}^{-1} x_{1}+x_{\bar{h}}^{-1} \bar{x}=d_{g^{\prime}}^{\prime} x_{g^{\prime}}+\sum_{g \in \mathfrak{C}} \bar{d}_{g} x_{g}=\sum_{g \in \mathfrak{C}} d_{g} x_{g}
$$

where $d_{g} \in\left\{0, \bar{d}_{g}, d_{g^{\prime}}^{\prime}, d_{g^{\prime}}^{\prime}+\bar{d}_{g}\right\} \in D_{C}^{-}$for all $g \in \mathfrak{C}$ and $d_{g} \triangleleft x$ by [DGH, Theorem 3.6]. The second subcase of $\tilde{C} \subset \bar{C}$ deals with $h \notin \bar{C}$ and we proceed exactly as in the second subcase of Case 1.

Recall, that $c p\left(x_{1}\right), c p(\bar{x})>1$ has been presumed at the intermediate stage of the proof where $x$ is assumed to be a general element of $D$ having a proper additive decomposition. Clearly, the case $c p\left(x_{1}\right)=c p(\bar{x})=1$ has already been treated when the theorem has been shown for $x \in F[G, \eta, \alpha]$. Thus, let us suppose for example that $c p\left(x_{1}\right)=1$ and $c p(\bar{x})>1$. Then, $x_{1}=a x_{g}$ with $a \in F^{\times}$and $g \in G$ which means $x_{\tilde{h}}^{-1} x_{1}=d_{e} x_{e}$ where $\tilde{h}=g$ and $d_{e}=x_{g}^{-1} a x_{g} \in F^{\times}$. Even though $x_{\tilde{h}}^{-1} x_{1}=d_{e} x_{e}$ is not a proper left representation we introduce formally $\tilde{C}=\{e\}$ such that $\tilde{C} \subset \bar{C}$ since $c p(\bar{x})>1$ and proceed as in Case 2.

Similar arguments yield that $x$ also has a right representation.

Theorem 6.2. With the assumptions as above, let $x \in D$ with $c p(x)>1$ such that $x_{\bar{h}}^{-1} x \in D_{\bar{C}}^{+}$for some $\bar{h} \in G, \bar{C} \in \mathcal{C}_{\leq}^{*}$ and with respect to this let $x_{\bar{h}}^{-1} x=\sum_{\bar{g} \in \overline{\mathfrak{C}}} \bar{d}_{\bar{g}} x_{\bar{g}}$ such that $\bar{d}_{\bar{g}} \in D_{\bar{C}}^{-}$for all $\bar{g} \in \overline{\mathfrak{C}}$ and $\overline{\bar{d}}_{\bar{g}} \neq 0$ for at least two different $\bar{g} \in \overline{\mathfrak{C}}$. Then the following hold true where the notation is as in the first part of Theorem 6.1:

(1) $C=\bar{C}$.

(2) $h C=\bar{h} C$.

(3) If $c \in C$ with $h=\bar{h} c$ and if $u_{g} \in F^{\times} X_{C^{\prime}}, g^{\prime} \in \mathfrak{C}$ with $x_{c} x_{g}=u_{g} x_{g^{\prime}}$ for all $g \in \mathfrak{C}$ then $\bar{d}_{g^{\prime}}=\alpha_{\bar{h}}^{-1}\left(\eta(\bar{h}, c)^{-1}\right) x_{c} d_{g} x_{c}^{-1} u_{g}$ for all $g \in \mathfrak{C}$.

(4) $\bar{d}_{g} \triangleleft x$ for all $g \in \mathfrak{C}$ and $x_{\bar{h}}^{-1} x=\sum_{g \in \mathfrak{C}} \bar{d}_{g} x_{g}$ is a left representation of $x$. 
Proof. (1): We begin with the special case $\bar{h}=e$ and assume $C \subset \bar{C}$. Then $C \subseteq \bar{C}^{\prime}$ and therefore $x_{h}^{-1} x \in D_{C}^{+} \subseteq D_{\bar{C}}^{-}$.

Case 1: $C_{h}^{+} \subset \bar{C}$. Then $C_{h}^{+} \subseteq \bar{C}^{\prime}$ and $x_{\bar{h}}^{-1} x=x=x_{h}\left(x_{h}^{-1} x\right) \in D_{\bar{C}}^{-}$. On the other hand $x_{\bar{h}}^{-1} x=\sum_{\bar{g} \in \overline{\mathfrak{C}}} \bar{d}_{\bar{g}} x_{\bar{g}} \in D_{\bar{C}}^{-}\left(\left(\bar{C} / \bar{C}^{\prime}, \eta, \alpha\right)\right)$ such that $\bar{d}_{\bar{g}} \neq 0$ for at least two different $\bar{g} \in \overline{\mathfrak{C}}$ which is a contradiction.

Case 2: $C_{h}^{+}=\bar{C}$, that is $C_{h}^{-}=\bar{C}^{\prime}$, and $x x_{h}^{-1}=x_{h}\left(x_{h}^{-1} x\right) x_{h}^{-1} \in D_{\bar{C}}^{-}$follows. There exist $h^{\prime} \in \overline{\mathfrak{C}}, u_{h} \in F^{\times} X_{\bar{C}^{\prime}}$ satisfying $x_{h}=u_{h} x_{h^{\prime}}$. Hence, $x_{\bar{h}}^{-1} x=x=\left(x x_{h}^{-1} u_{h}\right) x_{h^{\prime}}$ with $x x_{h}^{-1} u_{h} \in D_{\bar{C}}^{-}$is a power series representation of $x_{\bar{h}}^{-1} x$ which is a contradiction as in Case 1.

Case 3: $\bar{C} \subset C_{h}^{+}$. Then $\bar{C} \subseteq C_{h}^{-}$follows and therefore $x_{h}^{-1} x \in D_{C}^{+} \subseteq D_{h}^{-}$. Moreover, $x=x_{\bar{h}}^{-1} x \in D_{\bar{C}}^{+} \subseteq D_{h}^{-}$which implies $x_{h}^{-1}=\left(x_{h}^{-1} x\right) x^{-1} \in D_{h}^{-}$. Hence, $x_{e}$ and $x_{h}$ are linearly dependent over $D_{h}^{-}$, a contradition.

Similar arguments disprove $\bar{C} \subset C$ where it should be noted that $d_{g} \triangleleft x$ for $g \in \mathfrak{C}$ will not be used directly. Instead of this we apply the derived property $d_{g} \neq 0$ for at least two different $g \in \mathfrak{C}$. All this shows the first statement of the theorem for the case $\bar{h}=e$. Now, the general situation can be treated as follows. We put $x^{\prime}=x_{\bar{h}}^{-1} x$ with $x_{e}^{-1} x^{\prime}=\sum_{\bar{g} \in \overline{\mathfrak{C}}} \bar{d}_{\bar{g}} x_{\bar{g}}$ and obtain from $x_{h}^{-1} x=\sum_{g \in \mathfrak{C}} d_{g} x_{g}$ a left representation $x_{h^{\prime}}^{-1} x^{\prime}=\sum_{g \in \mathfrak{C}} d_{g}^{\prime} x_{g}$ where $h^{\prime}=\bar{h}^{-1} h$ and $d_{g}^{\prime}=\alpha_{h}^{-1}\left(\eta\left(\bar{h}, h^{\prime}\right)^{-1}\right) d_{g}$ for all $g \in \mathfrak{C}$.

(2): Again, we first suppose $\bar{h}=e$. Then Case 3 above shows that $C=\bar{C} \subset C_{h}^{+}$cannot hold true. Thus $h \in C$ follows which means $h C=\bar{h} C=C$. In the general situation we also define $x^{\prime}=x_{\bar{h}}^{-1} x$ and consider $x_{e}^{-1} x^{\prime}, x_{h^{\prime}}^{-1} x^{\prime}$. This provides $\bar{h}^{-1} h=h^{\prime} \in C$.

(3): Here we just have to transform the left representation $x_{h}^{-1} x=\sum_{g \in \mathfrak{C}} d_{g} x_{g}$ by means of $x_{h}^{-1}=x_{c}^{-1} \alpha_{\bar{h}}^{-1}(\eta(\bar{h}, c)) x_{\bar{h}}^{-1}$ into a formal power series representation of $x_{\bar{h}}^{-1} x$.

(4): This is a direct consequence of statement (3) and [DGH, Proposition 4.8] since $d_{g} \triangleleft x$ for all $g \in \mathfrak{C}$.

\section{Remarks.}

(1) Similar arguments can be used in order to prove a corresponding theorem for right representations.

(2) With the assumptions as above, let $H$ be a convex subgroup of $G$ and let $\leq$ also denote the Conradian left-order of $H$ induced by the left-order $\leq$ of $G$. Then, the rational closure $D_{H}$ of $F[H, \eta, \alpha]$ in $D$ is free with respect to $\leq$ since the convex subgroups of $H$ coincide with the convex subgroups of $G$ contained in $H$. We will apply our results to $D_{H}$ instead of $D$, that is, we replace $G$ by $H$. This means that for any $x \in D_{H}, x \notin F X_{H}$ there exist an $h \in H$, a convex subgroup $\bar{C}$ of $H$ which is a convex successor in $H$, and a left representation $x_{\bar{h}}^{-1} x=\sum_{\bar{g} \in \overline{\mathfrak{C}}} \bar{d}_{\bar{g}} x_{\bar{g}}$ of $x$ where any $\bar{d}_{\bar{g}}, \bar{g} \in \overline{\mathfrak{C}}$ is strictly simpler than $x$ where here simpler refers to the complexity which describes how an element of $D_{H}$ is built from elements in $F[H, \eta, \alpha]$. Thus, $\bar{d}_{\bar{g}} \neq 0$ holds true for at least two different $\bar{g} \in \overline{\mathfrak{C}}$. We now apply Theorem 6.2 and see that this left representation of $x$ 
as an element from $D_{H}$ is also a left representation of $x$ as an element from $D$ since $\bar{C}$ is a convex successor in $G$ as well. Thus, any $\bar{d} \bar{g}, \bar{g} \in \overline{\mathfrak{C}}$ is also strictly simpler than $x$ where now simpler refers to the complexity originally defined for the elements in $D$.

Definition 6.3. Let $x \in D$ such that $c p(x)>1$ and let $x_{h}^{-1} x=\sum_{g \in \mathfrak{C}} d_{g} x_{g}$ be as in Theorem 6.1. Then, $x$ is called reduced if $h C=C$, that is $h \in C$.

Because of Theorem 6.2 the definition of reduced does not depend on the given left representation of $x$. If $x \in D$ is reduced then there is always a uniquely determined left representation $x=\sum_{g \in \mathfrak{C}} d_{g} x_{g}$ which we will call the pure left representation of $x$ and which is also a right representation. Furthermore, for any $x \in D$ with $c p(x)>1$ there exist an $h \in G$ and a reduced $x^{\prime} \in D$ such that $x=x_{h} x^{\prime}$.

\section{Remarks about left representations of sums and products.}

The following remarks apply in a similar manner to right representations. If $x, y \in D$ are given then left representations of $x+y$ and $x \cdot y$ essentially result from adding and multiplying left representations of $x$ and $y$ respectively as described in the proof of Theorem 6.1. We shall give a more detailed explanation of this which will be needed in Section 8. To improve the readability we shall introduce left and right representations also for elements from $F X_{G}$. This will be done in an obvious way, that is, for $a x_{g} \in F X_{G}$ we call $a x_{g}$ itself a left and right representation and one shall always bear in mind that this does not have the proper meaning.

Left representations of sums.

If $x$ and $y$ are in $F X_{G}$ then a left representation of $x+y$ results obviously. Thus, let us assume $c p(x)>1$ or $c p(y)>1$. In the proof of Theorem 6.1 we have explained how to derive a left representation of $x=x_{1}+\bar{x}$ from those of $x$ and $\bar{x}$ and this method can almost completely be applied to $x, y$, and $x+y$. The first observation is that two essentially different cases have to be distinguished where the second case has two subcases:

A1: Up to exchanging the roles of $x$ and $y$ there exist $h \in G, C \in \mathcal{C}_{\leq}^{*}$, and $g \in \mathfrak{C}, g \neq e$ such that $x_{h}^{-1} x, x_{h}^{-1} y x_{g}^{-1} \in D_{C}^{-}$and

$$
x_{h}^{-1}(x+y)=x_{h}^{-1} x+\left(x_{h}^{-1} y x_{g}^{-1}\right) x_{g}
$$

is a left representation of $x+y$.

A2: Up to exchanging the roles of $x$ and $y$ there exist $h \in G$ and $C \in \mathcal{C}_{\leq}^{*}$ such that

$$
x_{h}^{-1} x=\sum_{g \in \mathfrak{C}} d_{g} x_{g} \text { and } x_{h}^{-1} y=\sum_{g \in \mathfrak{C}} d_{g}^{\prime} x_{g},
$$

where the left equation shows a proper left representation of $x$. If the power series given on the right side has more than one non-trivial summand then it is a proper left representation of $y$. However, it is also possible that only one non-zero summand exists. But in any case, $d_{g} \triangleleft x$ and $d_{g}^{\prime} \unlhd y$ hold true for all $g \in \mathfrak{C}$ and two subcases occur: 
A 2.1 There are at least two different $g \in \mathfrak{C}$ with $d_{g}+d_{g}^{\prime} \neq 0$. Then, Theorem 6.2 ensures that

$$
x_{h}^{-1}(x+y)=\sum_{g \in \mathfrak{C}}\left(d_{g}+d_{g}^{\prime}\right) x_{g}
$$

is a proper left representation of $x+y$.

A2.2 There is only one or no $g \in \mathfrak{C}$ with non-zero $d_{g}+d_{g}^{\prime}$. Then,

$$
x_{h}^{-1}(x+y)=\left(d_{g}+d_{g}^{\prime}\right) x_{g} \text { or } x+y=0 .
$$

In this case we do not obtain a proper left representation of $x+y$. However, in further investigations we will frequently use transfinite induction on the complexity of the elements of $D$ and then we will be able to apply even in this subcase the induction hypothesis to $x+y$.

\section{Left representations of products.}

For later use it will be enough to restrict our discussion to the case where $x$ and $y$ are reduced and $c p(x), c p(y)>1$. In the proof of Theorem 6.1 we have seen how to deduce a left representation of $x=x_{1} \cdot \bar{x}$ from those of $x$ and $\bar{x}$ and again, these ideas can almost completely be transferred to $x, y$, and $x \cdot y$. Some arguments are actually becoming simpler since $x$ and $y$ are assumed to be reduced. As in the proof of Theorem 6.1 there are three essentially different cases to be distinguished:

M1: There is a left representation $y=\sum_{g \in \mathfrak{C}} d_{g} x_{g}$ of $y$ such that $x \in D_{C}^{-}$. Then,

$$
x y=\sum_{g \in \mathfrak{C}}\left(x d_{g}\right) x_{g}
$$

is a left representation of $x y$.

M2: There is a left representation $x=\sum_{g \in \mathfrak{C}} d_{g} x_{g}$ of $x$ such that $y \in D_{C}^{-}$. Then,

$$
x y=\sum_{g \in \mathfrak{C}}\left(d_{g} x_{g} y x_{g}^{-1}\right) x_{g}
$$

is a left representation of $x y$.

M3: There are left representations $x=\sum_{g \in \mathfrak{C}} \bar{d}_{g} x_{g}$ and $y=\sum_{g \in \mathfrak{C}} \tilde{d}_{g} x_{g}$ of $x$ and $y$ respectively. Then,

$$
x y=\sum_{g \in \mathfrak{C}} d_{g} x_{g} \in D_{C}^{-}\left(\left(C / C^{\prime}, \eta, \alpha\right)\right),
$$

where

$$
d_{g} x_{g}=\sum_{\substack{\bar{g}, \tilde{g} \in \mathfrak{C} \\ \bar{g} \tilde{g} C^{\prime}=g C^{\prime}}} \bar{d}_{\bar{g}} x_{\bar{g}} \tilde{d}_{\tilde{g}} x_{\tilde{g}} \text { and } d_{g}=\sum_{\substack{\bar{g}, \tilde{g} \in \mathfrak{C} \\ \bar{g} \tilde{g} C^{\prime}=g C^{\prime}}} \bar{d}_{\bar{g}}\left(\left(x_{\bar{g}} \tilde{d}_{\tilde{g}} x_{\bar{g}}^{-1}\right) x_{\bar{g}} x_{\tilde{g}} x_{g}^{-1}\right)
$$

for all $g \in \mathfrak{C}$ such that each $d_{g}$ is a finite sum with summands which are products with two factors each, where the left factor is strictly simpler than $x$ and the right factor strictly simpler than $y$. If $d_{g}$ is non-zero for at least two $g \in \mathfrak{C}$ then $x y=\sum_{g \in \mathfrak{C}} d_{g} x_{g}$ is a left representation of $x y$. Otherwise, there exists a $g \in \mathfrak{C}$ such that $x y=d_{g} x_{g}$ with $d_{g}$ as above. This is no proper left representation in general but also this presentation will be needed in later applications. 


\section{The Vector Space of Formal Power Series}

In this section we summarize all propositions and theorems which refer to Dubrovin's method of embedding left-ordered groups into division rings and which will be used in this paper. They can be found for example in [D2, D3, D4] where, however, some proofs are kept rather short and also results we need are occasionally hidden or they occur as special cases in a broader context. The author of this paper is preparing a book which is devoted to Dubrovin's work on this subject. It will provide a comprehensive representation of his results about the embedding of left-ordered groups into division rings by means of formal power series where emphasis is placed on detailed proofs. This involves for example all propositions and theorems included in this section. The notation we use here is similar to that in [GS] and is also adapted to the subject of this paper. Therefore, it differ from what is introduced in [D2, [D3, D4].

Let $R=F[G, \eta, \alpha]$ be a crossed product of a group $G$ over a skew field $F$ where $G$ is endowed with a left-order $\leq$ which need not be Conradian. We also fix a basis $X_{G}=\left\{x_{g} \mid g \in G\right\}$ of the $F$-vector space $F[G, \eta, \alpha]$. A mapping

$$
m: G \longrightarrow F, g \longmapsto m_{g}
$$

is called a formal power series in $G$ over $F$ if its support $\operatorname{supp} m=\left\{g \in G \mid m_{g} \neq 0\right\}$ is well-ordered with respect to $\leq$. We write $m=\mathcal{O}$ in case of $m(g)=m_{g}=0$ for all $g \in G$. The set of all formal power series in $G$ over $F$ will be denoted by $F((G))$.

Definition 7.1. If $m \in F((G)), m \neq \mathcal{O}$ then $v(m)$ denotes the minimal element of $\operatorname{supp} m$.

For $m=\mathcal{O}$ we put $v(m)=\infty$ where $\infty$ has the obvious meaning.

Definition 7.2. A mapping $f: F((G)) \longrightarrow F((G))$ is said to be $v$-compatible if

$$
v(m) \leq v\left(m^{\prime}\right) \Longleftrightarrow v(f(m)) \leq v\left(f\left(m^{\prime}\right)\right)
$$

for all $m, m^{\prime} \in F((G))$.

In [D2] a $v$-compatible mapping is called monotone. For arbitrary $m, m^{\prime} \in F((G))$ and $a \in F$ we define $m+m^{\prime}$ and $m a$ as usual:

$$
\begin{gathered}
m+m^{\prime}: G \longrightarrow F, g \longmapsto m_{g}+m_{g}^{\prime}, \\
m a: G \longrightarrow F, g \longmapsto m_{g} a .
\end{gathered}
$$

These operations are well-defined and they turn $F((G))$ into a right $F$-vector space. The endomorphism ring $\operatorname{End}(F((G)))$ of $F((G))$ is a left $F$-vector space with respect to the usual operations, that is, $\operatorname{End}(F((G)))$ is a left $F$-algebra.

Proposition 7.3. (cf. [D2, Proposition 5.2]) Any v-compatible endomorphism of $F((G))$ is injective. The set of all v-compatible automorphisms of $F((G))$ is a subgroup of $\operatorname{Aut} F((G))$.

Later on we will see that there exists a naturally embedding of $F[G, \eta, \alpha]$ into the endomorphism ring of $F((G))$. This embedding depends on the basis $X_{G}$ that has been fixed above and for a different basis $X_{G}^{\prime}$ of $F[G, \eta, \alpha]$ we will get a different endomorphism ring and therefore another embedding of $F[G, \eta, \alpha]$. 
An element $m \in F((G))$ shall also be written as a formal sum $m=\sum x_{g} m_{g}$ such that

$$
\sum x_{g} m_{g}+\sum x_{g} m_{g}^{\prime}=\sum x_{g}\left(m_{g}+m_{g}^{\prime}\right),\left(\sum x_{g} m_{g}\right) a=\sum x_{g}\left(m_{g} a\right)
$$

for all $m, m^{\prime} \in F((G))$ and $a \in F$. In case of $\operatorname{supp} m=\left\{g_{1}, \ldots, g_{n}\right\}$ we also use the notation $m=x_{g_{1}} m_{g_{1}}+\cdots+x_{g_{n}} m_{g_{n}}$. To avoid any misunderstanding we occasionally write $F\left(\left(X_{G}\right)\right)$ and $F\left(\left(X_{G}^{\prime}\right)\right)$ instead of $F((G))$ if $F[G, \eta, \alpha]$ will be considered with respect to different bases $X_{G}$ and $X_{G}^{\prime}$ respectively.

Definition 7.4. A set $\left\{m_{i} \mid i \in I\right\} \subseteq F((G))$ of formal power series is said to be summable if for any $g \in G$ there is only a finite number of $i \in I$ such that $m_{i}(g) \neq 0$ and if the union of all corresponding supports $\operatorname{supp} m_{i}, i \in I$ is well-ordered.

If $\left\{m_{i} \mid i \in I\right\}$ is summable then $\sum_{i \in I} m_{i}(g)$ is a finite sum for any $g \in G$ such that $m_{g}:=\sum_{i \in I} m_{i}(g) \in F$ and the support of $m: G \longrightarrow F, g \longmapsto m_{g}$ is well-ordered. In this case we write $\sum_{i \in I} m_{i}=m \in F((G))$ and we obtain a new meaning of the representation of formal power series as formal sums. To see this, let $m=\sum x_{g} m_{g}$ be a non-zero element of $F((G))$. Considering $x_{g} m_{g}$ for $g \in \operatorname{supp} m$ as the formal power series with support $\{g\}$ which maps $g$ onto $m_{g}$ we immediately conclude that $\left\{x_{g} m_{g} \mid g \in \operatorname{supp} m\right\}$ is summable and

$$
\sum_{g \in \operatorname{supp} m} x_{g} m_{g}=m
$$

Lemma 7.5. Let $I, J$ be sets and for all $i \in I, j \in J$ let $m_{i j} \in F((G))$ such that the following conditions are satisfied:

(1) $\operatorname{supp} m_{i j}$ and $\operatorname{supp} m_{i j^{\prime}}$ are disjoint for all $i \in I$ and different $j, j^{\prime} \in J$,

(2) $\left\{m_{i j} \mid j \in J\right\}$ is summable for all $i \in I$,

(3) If $m_{i}=\sum_{j \in J} m_{i j}$ for all $i \in I$ then $\left\{m_{i} \mid i \in I\right\}$ is summable.

Furthermore, let $K$ be a set and let $M_{k} \subseteq I \times J$ for all $k \in K$ such that $M_{k} \cap M_{k^{\prime}}=\emptyset$ for $k \neq k^{\prime}$ and $I \times J=\bigcup_{k \in K} M_{k}$. Then, the following statements hold true:

(4) $\left\{m_{i j} \mid(i, j) \in M_{k}\right\}$ is summable for all $k \in K$,

(5) If $\bar{m}_{k}=\sum_{(i, j) \in M_{k}} m_{i j}$ for all $k \in K$ then $\left\{\bar{m}_{k} \mid k \in K\right\}$ is summable,

(6) $\sum_{i \in I} m_{i}=\sum_{k \in K} \bar{m}_{k}$.

Proof. We first observe that $M=\bigcup_{i \in I, j \in J} \operatorname{supp} m_{i j}$ is well-ordered. Indeed, because of assumption (1) and (2) for all $i \in I$ the support $\operatorname{supp} m_{i}$ coincides with the union of all $\operatorname{supp} m_{i j}, j \in J$ and assumption (3) yields the claim.

Next, we show statement (4). As a subset of $M$, the union of all $\operatorname{supp} m_{i j}$ with $(i, j) \in M_{k}$ is well-ordered. Now, let $g \in G$. We choose $\left(i_{1}, j_{1}\right), \ldots,\left(i_{l}, j_{l}\right) \in M_{k}$ where $i_{1}, \ldots, i_{l}$ are pairwise distinct and $m_{i_{1} j_{1}}(g), \ldots, m_{i_{l} j_{l}}(g)$ are non-zero. Assumption (1) then implies $m_{i_{1}}(g), \ldots, m_{i_{l}}(g) \neq 0$. According to condition (3) there is only a finite number of $i \in I$ such that $(i, j) \in M_{k}$ and $m_{i j}(g) \neq 0$ for some $j \in J$. Let these indices be given as $i_{1}, \ldots, i_{l}$. Because of assumption (2) for any $i \in\left\{i_{1}, \ldots, i_{l}\right\}$ there is only a finite number of $j \in J$ satisfying $m_{i j}(g) \neq 0$ (condition (1) even ensures that there is just one $j$ ). Hence, the number of all $(i, j) \in M_{k}$ with $m_{i j}(g) \neq 0$ is finite.

We turn to the proof of statement (5). The same arguments as used above provide that $\bigcup_{k \in K} \operatorname{supp} \bar{m}_{k}$ is well-ordered. We assume that there exist $g \in G$ and an infinite 
number of pairwise distinct $k_{1}, k_{2}, \cdots \in K$ such that $\bar{m}_{k_{1}}(g), \bar{m}_{k_{2}}(g), \ldots$ are not-trivial. Then, for any $l=1,2, \ldots$ there exists $\left(i_{l}, j_{l}\right) \in M_{k_{l}}$ with $m_{i_{l} j_{l}}(g) \neq 0$ and therefore $m_{i_{l}}(g) \neq 0$. We apply assumption (3) and conclude that there is an $i \in I$ and an infinite number of $j \in J$ satisfying $m_{i j}(g) \neq 0$. This contradicts condition (2).

In order to verify statement (6), let $g \in G$ with $\left\{i \in I \mid m_{i}(g) \neq 0\right\}=\left\{i_{1}, \ldots, i_{k}\right\}$. By assumption (1) for any $l=1, \ldots, k$ there exists exactly one $j_{l} \in J$ with $m_{i_{l} j_{l}}(g) \neq 0$, that is, $m_{i_{l}}(g)=m_{i_{l} j_{l}}(g)$. Thus, $m_{i_{1} j_{1}}(g)+\cdots+m_{i_{k} j_{k}}(g)$ is the image of $g$ with respect to $\sum_{i \in I} m_{i}$. On the other hand, for any $k \in K$ with $\bar{m}_{k}(g) \neq 0$ there exists $(i, j) \in M_{k}$ satisfying $m_{i j}(g) \neq 0$. Condition (1) implies $m_{i}(g) \neq 0$ which means $i \in\left\{i_{1}, \ldots, i_{k}\right\}$ and $j \in\left\{j_{1}, \ldots, j_{k}\right\}$. Hence, $\bar{m}_{k}(g)$ is the sum of all $m_{i_{l} j_{l}}(g)$ such that $\left(i_{l}, j_{l}\right) \in M_{k}$ and therefore $m_{i_{1} j_{1}}(g)+\cdots+m_{i_{k} j_{k}}(g)$ is also the image of $g$ with respect to $\sum_{k \in K} \bar{m}_{k}$.

Definition 7.6. An endomorphism $f$ of $F((G))$ is called continuous if the following condition is satisfied: If $\left\{m_{i} \mid i \in I\right\} \subseteq F((G))$ is summable then $\left\{f\left(m_{i}\right) \mid i \in I\right\}$ is summable and $f\left(\sum_{i \in I} m_{i}\right)=\sum_{i \in I} f\left(m_{i}\right)$.

Continuous endomorphisms are called $\sigma$-linear in [D2].

Proposition 7.7. (cf. [D2, Proposition 5.1]) An endomorphism $f$ of $F((G)$ ) is continuous if for any $m=\sum x_{g} m_{g} \in F((G))$ the set $\left\{f\left(x_{g} m_{g}\right) \mid g \in \operatorname{supp} m\right\}$ is summable and $f(m)=\sum_{g \in \operatorname{supp} m} f\left(x_{g} m_{g}\right)$. The set of all continuous endomorphisms of $F((G))$ is a subalgebra of $\operatorname{End}(F((G)))$.

Theorem 7.8. (cf. [D4, Lemma 3] and [S]) If $f$ is a continuous and $v$-compatible automorphism of $F((G))$ then $f^{-1}$ is also continuous.

Definition 7.9. A mapping $f: F((G)) \longrightarrow F((G))$ is called $v$-compatible on $G$ if

$$
g \leq g^{\prime} \Longleftrightarrow v\left(f\left(x_{g}\right)\right) \leq v\left(f\left(x_{g^{\prime}}\right)\right)
$$

for all $g, g^{\prime} \in G$. And $f$ is called surjective on $G$ if for any $g \in G$ there exists $g^{\prime} \in G$ such that $g=v\left(f\left(x_{g^{\prime}}\right)\right)$.

In [D2] the properties $v$-compatible on $G$ and surjective on $G$ are called locally monotone and locally surjective respectively.

Theorem 7.10. (cf. [D2, Theorem 5.1]) A continuous endomorphism $f$ of $F((G))$ is a $v$-compatible automorphism if and only if $f$ is surjective on $G$ and $v$-compatible on $G$.

Next, we explain how $R=F[G, \eta, \alpha]$ can be considered as a subring of the endomorphism ring of $F((G))$ where the assumptions are as stated above (further details can be found in $[\mathrm{G}, \mathrm{GS}, \mathrm{J}])$. At first, we observe that $F[G, \eta, \alpha]$ can be considered as a subgroup of $F((G))$ with respect to the addition since any $m=a_{g_{1}} x_{g_{1}}+\cdots+a_{g_{n}} x_{g_{n}} \in F[G, \eta, \alpha]$ can be written as $m=x_{g_{1}} m_{g_{1}}+\cdots+x_{g_{n}} m_{g_{n}}$ with $m_{g_{1}}, \ldots, m_{g_{n}} \in F$ which causes an isomorphism between $F[G, \eta, \alpha]$ and the additive group of all formal power series with finite support. Occasionally, it is convenient to interpret power series with finite supports as elements from $F[G, \eta, \alpha]$ in order to apply the multiplication given in $F[G, \eta, \alpha]$. We will use this frequently without any further explanation. 
Let us return to the problem of embedding $R=F[G, \eta, \alpha]$ into $\operatorname{End}(F((G)))$. If $m=\sum x_{g} m_{g}$ is an arbitrary element of $F((G))$ then $\{h g \mid g \in \operatorname{supp} m\}$ is well-ordered for any $h \in G$ since $\leq$ is a left-order of $G$. This ensures that

$$
\iota_{a_{h} x_{h}}: F((G)) \longrightarrow F((G)), \sum x_{g} m_{g} \longmapsto \sum x_{h g}\left(\alpha_{h g}^{-1}\left(a_{h} \eta(h, g)\right) m_{g}\right)
$$

is a well-defined mapping. According to the remark above, it should always be noted that

$$
x_{h g}\left(\alpha_{h g}^{-1}\left(a_{h} \eta(h, g)\right) m_{g}\right)=a_{h} x_{h} x_{g} m_{g}
$$

for arbitrary $g, h \in G$ and $m_{g}, a_{h} \in F$ which yields

$$
\iota_{a_{h} x_{h}} \circ \iota_{a_{h^{\prime}}^{\prime} x_{h^{\prime}}}=\iota_{a_{h} x_{h} a_{h^{\prime}}^{\prime} x_{h^{\prime}}}
$$

for all $h, h^{\prime} \in G$ and $a_{h}, a_{h^{\prime}}^{\prime} \in F$. If $a_{h} \neq 0$ then $\iota_{a_{h} x_{h}}$ is a continuous and $v$-compatible automorphism of $F((G))$ with $\iota_{a_{h} x_{h}}\left(x_{g} F\right) \subseteq x_{h g} F$ for all $g \in G$. Following [D2, D3], we call endomorphisms with this property monomial. For pairwise distinct $h_{1}, \ldots, h_{n} \in G$ and arbitrary $a_{h_{1}}, \ldots, a_{h_{n}} \in F$ we now define

$$
\iota_{a_{h_{1}} x_{h_{1}}+\cdots+a_{h_{n}} x_{h_{n}}}: F((G)) \longrightarrow F((G)), m \longmapsto \iota_{a_{h_{1} x_{h_{1}}}}(m)+\cdots+\iota_{a_{h_{n} x_{h_{n}}}}(m) .
$$

Clearly, $\iota_{a_{h_{1}} x_{h_{1}}+\cdots+a_{h_{n} x_{h_{n}}}}$ is an endomorphism of $F((G))$ which in addition is continuous because of Proposition 7.7. A straightforward calculation shows that

$$
\iota: F[G, \eta, \alpha] \longrightarrow \operatorname{End}(F((G))), a_{h_{1}} x_{h_{1}}+\cdots+a_{h_{n}} x_{h_{n}} \longmapsto \iota_{a_{h_{1}} x_{h_{1}}+\cdots+a_{h_{n}} x_{h_{n}}}
$$

is an injective ring homomorphism. Hence, with respect to the composition $\iota(\mathcal{G})$ is a group of monomial, $v$-compatible, and continuous automorphisms of $F((G))$.

For the sake of simplicity, we shall not distinguish between the elements of $F[G, \eta, \alpha]$ and their images under $\iota$. It will be clear from the context whether $a_{h_{1}} x_{h_{1}}+\cdots+a_{h_{n}} x_{h_{n}}$ is an element from $F[G, \eta, \alpha]$ or $F((G))$ or whether it is the endomorphism of $F((G))$ which maps $m=\sum x_{g} m_{g}$ onto $a_{h_{1}} x_{h_{1}} m+\cdots+a_{h_{n}} x_{h_{n}} m$ where $a_{h_{i}} x_{h_{i}} m=\sum a_{h_{i}} x_{h_{i}} x_{g} m_{g}$ for $i=1, \ldots, n$. Therefore, we also consider $\mathcal{G}$ as a group of monomial, $v$-compatible, and continuous automorphisms of $F((G))$.

Proposition 7.11. (cf. [D1, D2, D3]) Let the notation be as above. Then, any nonzero element from $F[G, \eta, \alpha]$ - regarded as an endomorphism of $F((G))$ - is a continuous and $v$-compatible automorphism.

Proof. We apply Proposition 8.1 of [D2]. Let $\mathcal{G}_{h}=\left\{a_{g} x_{g} \in \mathcal{G} \mid v\left(a_{g} x_{g} x_{h}\right)=h\right\}$ where $h \in G$. Clearly, $v\left(a_{g} x_{g} x_{h}\right)=g h$ implies $\mathcal{G}_{h}=\left\{a x_{e} \mid a \in F^{\times}\right\}$and we obtain for $a_{1} x_{e}, \ldots, a_{n} x_{e} \in \mathcal{G}_{h}$ with

$$
\mathcal{O}=\left(a_{1} x_{e}+\cdots+a_{n} x_{e}\right)\left(x_{h}\right)=a_{1} x_{e} x_{h}+\ldots a_{n} x_{e} x_{h}=\left(a_{1}+\cdots+a_{n}\right) x_{h}
$$

that $a_{1}+\cdots+a_{n}=0$ and therefore $a_{1} x_{e}+\cdots+a_{n} x_{e}=\mathcal{O}$. This proves the claim since $F[G, \eta, \alpha]$ is the subring of $\operatorname{End}(F((G)))$ generated by $\mathcal{G}$. 
Definition 7.12. Let $F[G, \eta, \alpha]$ be a crossed product of $G$ over the skew field $F$ with respect to the basis $X_{G}$ and let $\leq$ be a left-order of $G$. The rational closure $F(G, \eta, \alpha)$ of $F[G, \eta, \alpha]$ in $\operatorname{End}(F((G))$ ) (the rational closure of $\mathcal{G}$ in $\operatorname{End}(F((G)))$ ) is called the Dubrovin-quotient ring of $F[G, \eta, \alpha]$ (with respect to $\leq$ and $X_{G}$ ). If $F(G, \eta, \alpha)$ is a division ring then $F(G, \eta, \alpha)$ is called the Dubrovin-division ring of fractions of $F[G, \eta, \alpha]$.

Proposition 7.13. Let $R=F[G, \eta, \alpha]$ be a crossed product of $G$ over the skew field $F$ with respect to the basis $X_{G}$ and let $\leq$ be a left-order of $G$. Further, let $\varphi: R \longrightarrow R$ be a ring automorphism. If $D$ is the Dubrovin-quotient ring of $F[G, \eta, \alpha]$ with respect to $\leq$ and $X_{G}$ and if $D^{\prime}$ is the Dubrovin-quotient ring of $F[G, \eta, \alpha]$ with respect to $\leq$ and $X_{G}^{\prime}=\left\{x_{g}^{\prime} \mid g \in G\right\}$ where $x_{g}^{\prime}=\varphi\left(x_{g}\right)$ for all $g \in G$ then there exists a unique ring isomorphism $\psi: D \longrightarrow D^{\prime}$ which extends $\varphi$.

Proof. The uniqueness of $\psi$ can easily be proved by transfinite induction on the complexity $c p(d), d \in D$ which describes how $d$ is built in $\operatorname{End}(F((G)))$ from elements of $\mathcal{G}$. Since $\varphi(F)=F$ by Proposition 3.3 we obtain that

$$
\rho: F\left(\left(X_{G}\right)\right) \longrightarrow F\left(\left(X_{G}^{\prime}\right)\right), \sum x_{g} m_{g} \longmapsto \sum x_{g}^{\prime} \varphi\left(m_{g}\right)=\sum \varphi\left(x_{g}\right) \varphi\left(m_{g}\right)
$$

is a group isomorphism between the addivite groups $F\left(\left(X_{G}\right)\right)$ and $F\left(\left(X_{G}^{\prime}\right)\right)$ such that $\rho(m a)=\rho(m) \varphi(a)$ for all $m \in F\left(\left(X_{G}\right)\right)$ and $a \in F$. A straightforward calculation shows that

$$
\tau: \operatorname{End}\left(F\left(\left(X_{G}\right)\right)\right) \longrightarrow \operatorname{End}\left(F\left(\left(X_{G}^{\prime}\right)\right)\right), f \longmapsto \rho f \rho^{-1}
$$

is a ring isomorphism such that $\tau\left(a_{h} x_{h}\right)=\varphi\left(a_{h} x_{h}\right)$ for all $h \in G$ and $a_{h} \in F$. In addition, $\tau$ and $\varphi$ coincide on $F[G, \eta, \alpha]$ and therefore $\tau$ maps the Dubrovin-quotient ring $D$ of $F[G, \eta, \alpha]$ with respect to $\leq$ and $X_{G}$ onto the Dubrovin-quotient ring $D^{\prime}$ of $F[G, \eta, \alpha]$ with respect to $\leq$ and $X_{G}^{\prime}$. The restriction $\psi$ of $\tau$ to $D$ is the desired isomorphism.

The following lemma occurs in [D2, D3, Proposition 7.1] in a slightly modified version. For the sake of completeness we add Dubrovin's proof.

Lemma 7.14. Let $R=F[G, \eta, \alpha]$ be a crossed product of the left-ordered group $G$ over the skew field $F$ and let $U$ be a subgroup of $G$. If $D_{U}$ denotes the rational closure of $F[U, \eta, \alpha]$ in $\operatorname{End}(F((G)))$ then

$$
\operatorname{supp} f(m) \subseteq\{g h \mid g \in U, h \in \operatorname{supp} m\}
$$

for all $f \in D_{U}$ and $m \in F((G))$.

Proof. Let the notation be as above, especially let $\operatorname{End}(F((G)))$ be defined with respect to the fixed basis $X_{G}$ of $F[G, \eta, \alpha]$. We prove the lemma by transfinite induction on the complexity $c p(f), f \in D_{U}$ which describes how $f$ is built in $\operatorname{End}(F((G)))$ from elements of $\mathcal{U}=F^{\times} X_{U}$. The statement is obvious in case of $f=\mathcal{O}$ or $f \in \mathcal{U}$. Thus, let $c p(f)>1$. If $f$ is additively decomposable then there exist $f_{1}, \ldots, f_{n} \in D_{U}$ such that $f_{1}, \ldots, f_{n} \triangleleft f$ and $f=f_{1}+\cdots+f_{n}$. Now, the claim follows immediately by $\operatorname{supp} f(m) \subseteq \operatorname{supp} f_{1}(m) \cup \cdots \cup \operatorname{supp} f_{n}(m)$ and the induction hypothesis. Next, let $f$ be additively indecomposable. Then, $f$ is a unit in $\operatorname{End}(F((G)))$ by [DGH, Theorem 4.9] 
and therefore in $D_{U}$ where $f^{-1} \triangleleft f$. There exist uniquely determined $m_{1}, m_{2} \in F((G))$ with $f(m)=m_{1}+m_{2}$ such that

$$
\operatorname{supp} m_{1} \subseteq\{g h \mid g \in U, h \in \operatorname{supp} m\} \text { and } \operatorname{supp} m_{2} \cap\{g h \mid g \in U, h \in \operatorname{supp} m\}=\emptyset
$$

where $m_{2}=\mathcal{O}$ must be shown. Clearly, $f^{-1}\left(m_{2}\right)=m-f^{-1}\left(m_{1}\right)$ and the induction hypothesis imply supp $f^{-1}\left(m_{2}\right) \subseteq\{g h \mid g \in U, h \in \operatorname{supp} m\}$. Now, let us assume that $m_{2} \neq \mathcal{O}$. Then, supp $f^{-1}\left(m_{2}\right) \neq \emptyset$ and therefore

$$
\left\{g h \mid g \in U, h \in \operatorname{supp} m_{2}\right\} \cap\{g h \mid g \in U, h \in \operatorname{supp} m\} \neq \emptyset .
$$

Hence, there are $g, g^{\prime} \in U, h \in \operatorname{supp} m_{2}$, and $h^{\prime} \in \operatorname{supp} m$ such that $g h=g^{\prime} h^{\prime}$. This yields the contradiction

$$
\operatorname{supp} m_{2} \cap\{g h \mid g \in U, h \in \operatorname{supp} m\} \neq \emptyset .
$$

The next corollary is a direct consequence of Dubrovin's lemma and appears in [J].

Corollary 7.15. Let $R=F[G, \eta, \alpha]$ be a crossed product of a left-ordered group $G$ over a skew field $F$ with respect to the basis $X_{G}$ and let $F(G, \eta, \alpha)$ be the Dubrovinquotient ring of $F[G, \eta, \alpha]$ with respect to $\leq$ and $X_{G}$. If $F(G, \eta, \alpha)$ is a division ring then $F(G, \eta, \alpha)$ is strongly Huges-free.

Proof. Let $H$ be an arbitrary subgroup of $G$ with normal subgroup $N$ and let $h_{1}, \ldots, h_{n} \in H, n \in \mathbb{N}$ are given such that $h_{1} N, \ldots, h_{n} N$ are pairwise different. It has to be shown that the mappings $x_{h_{1}}, \ldots, x_{h_{n}}$ are linearly independent over the rational closure $D_{N}$ of $F[N, \eta, \alpha]$ in $F(G, \eta, \alpha)$. Thus, let $d_{1} x_{h_{1}}+\cdots+d_{n} x_{h_{n}}$ be the zero-map for arbitrarily given $d_{1}, \ldots, d_{n} \in D_{N}$. Then $d_{1} x_{h_{1}}\left(x_{e}\right)+\cdots+d_{n} x_{h_{n}}\left(x_{e}\right)=\mathcal{O}$ which means $d_{1}\left(x_{h_{1}}\right)+\cdots+d_{n}\left(x_{h_{n}}\right)=\mathcal{O}$. Since $N h_{1}, \ldots, N h_{n}$ are pairwise disjoint subsets of $G$ we conclude that $d_{i}\left(x_{h_{i}}\right), i=1, \ldots, n$ have disjoint supports by Lemma 7.14. Thus, $d_{i}\left(x_{h_{i}}\right)=\mathcal{O}$ and therefore $d_{i}=\mathcal{O}$ for all $i=1, \ldots, n$.

\section{Free Division Rings of Fractions and Power Series}

We shall use the notation as introduced in the sections before.

Theorem 8.1. Let $R=F[G, \eta, \alpha]$ be a crossed product of a locally indicable group $G$ over a skew field $F$ with respect to the basis $X_{G}$ and let $\leq$ be a Conradian left-order of $G$. Furthermore, let $D$ be a division ring of fractions of $R$ which is free with respect to $\leq$ and let $\mathcal{R}$ be the Dubrovin-quotient ring of $F[G, \eta, \alpha]$ with respect to $\leq$ and $X_{G}$. Then, there exists a ring isomorphism $\psi: D \longrightarrow \mathcal{R}$ which is the identity on $F[G, \eta, \alpha]$. Especially, $\mathcal{R}$ is a division ring and $D$ is strongly Hughes-free.

Corollary 8.2. Let $R=F[G, \eta, \alpha]$ be a crossed product of a locally indicable group $G$ over a skew field $F$ with respect to the basis $X_{G}$ and let $D, D^{\prime}$ be two division rings of fractions of $R$ which are free with respect to two Conradian left-orders $\leq, \leq$ respectively. Any ring automorphism $\varphi$ of $R$ can be extended to a ring isomorphism $\psi: D \longrightarrow D^{\prime}$. 
Proof. By Theorem 8.1 we may assume that $D=\mathcal{R}$ where $\mathcal{R}$ is the Dubrovin-division ring of fractions of $F[G, \eta, \alpha]$ with respect to $\leq$ and $X_{G}$. Furthermore, by Theorem 8.1 , Propositon 5.6. and Proposition 5.4 we may also assume that $D^{\prime}=\mathcal{R}^{\prime}$ where $\mathcal{R}^{\prime}$ is the Dubrovin-division ring of fractions of $F[G, \eta, \alpha]$ with respect to $\leq$ and $X_{G}^{\prime}=\varphi\left(X_{G}\right)$. It remains to apply Proposition 7.13 .

Remark. Theorem 8.1 is proved in [J] under the special assumption that $\leq$ has maximal rank. Furthermore, in [J] it is also shown that Hughes-freeness and strongly Hughes-freeness coincide which solves P.A. Linnell's Problem 4.8 from (cf. [L]). Here it is a consequence of Theorem 2.13, Proposition 2.10, Proposition 5.6, and Theorem 8.1. Our results also provide the following

Corollary 8.3. Let $R=F[G, \eta, \alpha]$ be a crossed product of a locally indicable group $G$ over a skew field $F$ and let $D$ be a division ring of fractions of $R$. Then $D$ is (strongly) Hughes-free if and only if $D$ is free with respect to at least one Conradian left-order of $G$. In this case, $D$ is free with respect to any Conradian left-order of $G$.

By means of $[\mathrm{JL}]$ we also obtain

Corollary 8.4. Let $G$ be a locally indicable group and let $F$ be a commutative field of characteristic zero. Then for any Conradian left-order the Dubrovin-quotient ring of the group ring $F[G]$ is a division ring.

As we will explain at the end of this section, Theorem 8.1 is an immediate consequence of Lemma 8.6 below. The notation we use is the same as in Theorem 8.1 and Section 4 where Definition 4.2 is of particular importance. The complexity $c p(d)$ for some $d \in D$ always refers to the manner how $d$ is built from elements of $\mathcal{G}=F^{\times} G$.

Lemma 8.5. Let $\lambda \in \Lambda$ and let $\psi_{\lambda}: D_{\leq \lambda} \longrightarrow \mathcal{R}$ be a mapping such that the following conditions are fulfilled:

(1) $\psi_{\lambda}(x)=x$ for all $x \in F[G, \eta, \alpha]$.

(2) $\psi_{\lambda}(x)$ is a continuous endomorphism of $F((G))$ for all $x \in D_{\leq \lambda}$.

(3) If $x \in D_{\leq \lambda}$ is reduced with $c p(x)>1$ and if $x=\sum_{g \in \mathfrak{C}} d_{g} x_{g}$ is the pure left representation of $x$ then $\left(\psi_{\lambda}(x)\right)\left(x_{e}\right)=\sum_{g \in \mathfrak{C}}\left(\psi_{\lambda}\left(d_{g}\right) x_{g}\right)\left(x_{e}\right) \in F((C))$.

(4) $\psi_{\lambda}\left(x_{\tilde{g}} x\right)=x_{\tilde{g}} \psi_{\lambda}(x)$ and $\psi_{\lambda}\left(x x_{\tilde{g}}\right)=\psi_{\lambda}(x) x_{\tilde{g}}$ for all $x \in D_{\leq \lambda}$ and all $\tilde{g} \in G$.

Then the following hold true:

(5) If $x \in D_{\leq \lambda}, c p(x)>1$ is arbitrary and if $x_{h}^{-1} x=\sum_{g \in \mathfrak{C}} d_{g} x_{g}$ is an arbitrary left representation of $x$ then $\left(\psi_{\lambda}(x)\right)\left(x_{e}\right)=x_{h} \sum_{g \in \mathfrak{C}}\left(\psi_{\lambda}\left(d_{g}\right) x_{g}\right)\left(x_{e}\right) \in x_{h} F((C))$.

(6) If $H$ is a convex subgroup of $G$ and $D_{H}$ the rational closure of $F[H, \eta, \alpha]$ in $D$ then $\left(\psi_{\lambda}(x)\right)\left(x_{e}\right) \in F((H))$ for any $x \in D_{\leq \lambda}, c p(x)>1$ which is in $D_{H}$. Especially, $\left(\psi_{\lambda}\left(d_{g}\right)\right)\left(x_{e}\right) \in F\left(\left(C^{\prime}\right)\right)$ for all $d_{g}$ occurring as a coefficient of a left representation of $x$ as given in (5).

(7) If $x \in D_{\leq \lambda}, c p(x)>1$ is arbitrary and if $x_{h}^{-1} x=\sum_{g \in \mathfrak{C}} d_{g} x_{g}$ is an arbitrary left representation of $x$ then $\left(\psi_{\lambda}(x)\right)\left(x_{\bar{g}}\right)=x_{h} \sum_{g \in \mathfrak{C}}\left(\psi_{\lambda}\left(d_{g}\right) x_{g}\right)\left(x_{\bar{g}}\right) \in x_{h} F((C))$ for all $\bar{g} \in C$. 
(8) If $x \in D_{\leq \lambda}, c p(x)>1$ is arbitrary and if $x_{h}^{-1} x=\sum_{g \in \mathfrak{C}} d_{g} x_{g}$ is an arbitrary left representation of $x$ then $\left(\psi_{\lambda}(x)\right)(m)=x_{h} \sum_{g \in \mathfrak{C}}\left(\psi_{\lambda}\left(d_{g}\right) x_{g}\right)(m) \in x_{h} F((C))$ for all $m \in F((C))$.

\section{Remarks.}

(1) Assumption (3) includes that $\left\{\left(\psi_{\lambda}\left(d_{g}\right) x_{g}\right)\left(x_{e}\right) \mid g \in \mathfrak{C}\right\}$ is summable.

(2) The appearance of an infinite sum in the statements above always include that this sum actually exists.

(3) Proposition 4.3 and $d_{g} \triangleleft x$ imply $d_{g} \in D_{\leq \lambda}$ for any $d_{g}$ which occurs as a coefficient of a left representation of $x$ as given above.

Proof. In order to prove statement (5) we first observe that $x_{h}^{-1} x$ is reduced with pure left representation $x_{h}^{-1} x=\sum_{g \in \mathfrak{C}} d_{g} x_{g}$. Clearly, $x_{h}^{-1} x \in D_{\leq \lambda}$ and therefore $\left(\psi_{\lambda}\left(x_{h}^{-1} x\right)\right)\left(x_{e}\right)=\sum_{g \in \mathfrak{C}}\left(\psi_{\lambda}\left(d_{g}\right) x_{g}\right)\left(x_{e}\right) \in F((C))$ by assumption (3). We now apply assumption (4) and conclude $\left(\psi_{\lambda}(x)\right)\left(x_{e}\right)=\left(\psi_{\lambda}\left(x_{h}\left(x_{h}^{-1} x\right)\right)\right)\left(x_{e}\right)=x_{h}\left(\psi_{\lambda}\left(x_{h}^{-1} x\right)\right)\left(x_{e}\right)$ which completes the proof of statement (5). Furthermore, statement (6) follows from statement (5) and remark (2) after Theorem 6.2. We show statement (7). For any $g \in \mathfrak{C}$ there are $g^{\prime} \in \mathfrak{C}$ and $u_{g} \in F^{\times} X_{C^{\prime}}$ with $x_{g} x_{\bar{g}}=u_{g} x_{g^{\prime}}$. Since $g \bar{g} C^{\prime}=\bar{g} g C^{\prime}=g^{\prime} C^{\prime}$ for all $g \in \mathfrak{C}$ the set $\left\{g^{\prime} \mid g \in \mathfrak{C}\right.$ and $\left.d_{g} \neq \mathcal{O}\right\}$ is well-ordered with respect to $\leq$. This means that $x_{h}^{-1}\left(x x_{\bar{g}}\right)=\sum_{g \in \mathfrak{C}}\left(d_{g} u_{g}\right) x_{g^{\prime}}$ is a left representation of $x x_{\bar{g}} \in D_{\leq \lambda}$ and statement (5) yields $\left(\psi_{\lambda}\left(x x_{\bar{g}}\right)\right)\left(x_{e}\right)=x_{h} \sum_{g \in \mathfrak{C}}\left(\psi_{\lambda}\left(d_{g} u_{g}\right) x_{g^{\prime}}\right)\left(x_{e}\right) \in x_{h} F((C))$. Assumption (4) provides $\left(\psi_{\lambda}\left(d_{g} u_{g}\right) x_{g^{\prime}}\right)\left(x_{e}\right)=\left(\psi_{\lambda}\left(d_{g} u_{g} x_{g^{\prime}}\right)\right)\left(x_{e}\right)=\left(\psi_{\lambda}\left(d_{g}\right) x_{g}\right)\left(x_{\bar{g}}\right)$ and $\left(\psi_{\lambda}\left(x x_{\bar{g}}\right)\right)\left(x_{e}\right)=\left(\psi_{\lambda}(x)\right)\left(x_{\bar{g}}\right)$ which proves statement $(7)$. In order to derive statement (8) let $m=\sum_{\bar{g} \in C} x_{\bar{g}} m_{\bar{g}}$ be an arbitrary element of $F((C))$. Assumption (2) ensures that $\psi_{\lambda}(x)$ is continuous and therefore $\left(\psi_{\lambda}(x)\right)(m)=\sum_{\bar{g} \in C}\left(\psi_{\lambda}(x)\right)\left(x_{\bar{g}} m_{\bar{g}}\right)$. Thus,

$$
\left(\psi_{\lambda}(x)\right)(m)=\sum_{\bar{g} \in C} x_{h} \sum_{g \in \mathfrak{C}}\left(\psi_{\lambda}\left(d_{g}\right) x_{g}\right)\left(x_{\bar{g}} m_{\bar{g}}\right)
$$

because of statement (7). By means of $d_{g} \in D_{\leq \lambda}$ and assumption (4) as well as $d_{g} \in D_{C}^{-}$ and statement (6) we conclude

$$
\left(\psi_{\lambda}\left(d_{g}\right) x_{g}\right)\left(x_{\bar{g}} m_{\bar{g}}\right)=\left[x_{g} x_{\bar{g}}\left(\psi_{\lambda}\left(x_{\bar{g}}^{-1} x_{g}^{-1} d_{g} x_{g} x_{\bar{g}}\right)\right)\left(x_{e}\right)\right] m_{\bar{g}} \in x_{g} x_{\bar{g}} F\left(\left(C^{\prime}\right)\right) .
$$

This shows that for a fixed $\bar{g} \in C$ the sets $\operatorname{supp}\left(\psi_{\lambda}\left(d_{g}\right) x_{g}\right)\left(x_{\bar{g}} m_{\bar{g}}\right), g \in \mathfrak{C}$ are pairwise disjoint. We apply Lemma 7.5 and obtain

$$
\begin{aligned}
\left(\psi_{\lambda}(x)\right)(m) & =\sum_{\bar{g} \in C} x_{h} \sum_{g \in \mathfrak{C}}\left(\psi_{\lambda}\left(d_{g}\right) x_{g}\right)\left(x_{\bar{g}} m_{\bar{g}}\right)=x_{h} \sum_{g \in \mathfrak{C}} \sum_{\bar{g} \in C}\left(\psi_{\lambda}\left(d_{g}\right) x_{g}\right)\left(x_{\bar{g}} m_{\bar{g}}\right) \\
& =x_{h} \sum_{g \in \mathfrak{C}}\left(\psi_{\lambda}\left(d_{g}\right) x_{g}\right)\left(\sum_{\bar{g} \in C} x_{\bar{g}} m_{\bar{g}}\right)=x_{h} \sum_{g \in \mathfrak{C}}\left(\psi_{\lambda}\left(d_{g}\right) x_{g}\right)(m) .
\end{aligned}
$$

As we have seen above, $\left(\psi_{\lambda}\left(d_{g}\right) x_{g}\right)\left(x_{\bar{g}} m_{\bar{g}}\right) \in x_{g} x_{\bar{g}} F\left(\left(C^{\prime}\right)\right) \subseteq F((C))$ for all $g \in \mathfrak{C}$, $\bar{g} \in C$ and we conclude $\left(\psi_{\lambda}(x)\right)(m) \in x_{h} F((C))$ which completes the proof.

Now, we are in the position to prove 
Lemma 8.6. For all $\lambda \in \Lambda$ there exists a mapping $\psi_{\lambda}: D_{\leq \lambda} \longrightarrow \mathcal{R}$ such that the following hold true:

(1) If $\lambda^{\prime} \in \Lambda$ with $\lambda^{\prime}<\lambda$ then $\psi_{\lambda}(x)=\psi_{\lambda^{\prime}}(x)$ for all $x \in D_{\leq \lambda^{\prime}}$.

(2) $\psi_{\lambda}(x)=x$ for all $x \in F[G, \eta, \alpha]$.

(3) $\psi_{\lambda}$ is additive on $D_{<\lambda}$.

(4) If $a \in D_{\leq \lambda}$ is a proper atom and $\lambda^{\prime}=c p(a)$ then the restriction of $\psi_{\lambda}$ to $D_{<\lambda^{\prime}}$ is an injective ring homomorphism.

(5) $\psi_{\lambda}(x)$ is a continuous and $v$-compatible automorphism of $F((G))$ for all nonzero $x \in D_{\leq \lambda}$.

(6) If $x \in D_{\leq \lambda}$ is reduced with $c p(x)>1$ and if $x=\sum_{g \in \mathfrak{C}} d_{g} x_{g}$ is the pure left representation of $x$ then $\left(\psi_{\lambda}(x)\right)\left(x_{e}\right)=\sum_{g \in \mathfrak{C}}\left(\psi_{\lambda}\left(d_{g}\right) x_{g}\right)\left(x_{e}\right) \in F((C))$.

(7) $\psi_{\lambda}\left(x_{\tilde{g}} x\right)=x_{\tilde{g}} \psi_{\lambda}(x)$ and $\psi_{\lambda}\left(x x_{\tilde{g}}\right)=\psi_{\lambda}(x) x_{\tilde{g}}$ for all $x \in D_{\leq \lambda}$ and $\tilde{g} \in G$.

Proof. We prove Lemma 8.6 by transfinite induction on $\lambda \in \Lambda$ and consider the case $\lambda=1$ first. Then, $D_{\leq \lambda}=F[G, \eta, \alpha]$ and we choose $\psi_{1}$ as the identity map such that statements (1),(2),(3),(4), and (7) are obviously true. Clearly, statement (5) follows by Proposition [7.11. To prove statement (6) we first choose a left representation of $x$ as given in the proof of Theorem 6.1 which is a finite sum $x_{h}^{-1} x=\sum_{g \in \mathfrak{C}} d_{g} x_{g}$ with $h \in C$ since $x$ is reduced and $d_{g} \in F\left[C^{\prime}, \eta, \alpha\right]$ for all $g \in \mathfrak{C}$. We now transform this left representation into the pure left representation of $x$ as explained before. Thus $h=e$ can be assumed, that is, $\psi_{1}(x)\left(x_{e}\right)=x=\sum_{g \in \mathfrak{C}} d_{g} x_{g}=\sum_{g \in \mathfrak{C}}\left(\psi_{1}\left(d_{g}\right) x_{g}\right)\left(x_{e}\right) \in F((C))$. Now, let $x \in D_{\leq \lambda}$ where $\lambda>1$. If there exists $\lambda^{\prime} \in \Lambda$ with $\lambda^{\prime}<\lambda$ such that $x \in D_{\leq \lambda^{\prime}}$ then we define $\psi_{\lambda}(x)=\psi_{\lambda^{\prime}}(x)$. Clearly, this definition does not depend on $\lambda^{\prime}, \lambda^{\prime}<\lambda$ and statements (1) and (2) are obviously true. Even though we have defined $\psi_{\lambda}(x)$ just in this special case we are already in the position to prove statement (3). Thus, let $x, y \in D_{<\lambda}$. Because of Proposition 4.3 there exists $\lambda^{\prime} \in \Lambda$ with $\lambda^{\prime}<\lambda$ such that $x, y, x+y \in D_{\leq \lambda^{\prime}}$. Therefore, $\psi_{\lambda}(x), \psi_{\lambda}(y)$, and $\psi_{\lambda}(x+y)$ are defined and we can apply the induction hypothesis to $\lambda^{\prime}$. Then, conditions (1)-(4) of Lemma 8.5 are fulfilled where $\lambda$ is replaced by $\lambda^{\prime}$ and statements (5)-(8) of Lemma 8.5 can be used for $\lambda^{\prime}$. With this in mind we now prove:

A: $\psi_{\lambda}(x+y)=\psi_{\lambda}(x)+\psi_{\lambda}(y)$ for all $x, y \in D_{<\lambda}$.

We assume that there exist $x, y \in D_{<\lambda}$ such that $\psi_{\lambda}(x+y) \neq \psi_{\lambda}(x)+\psi_{\lambda}(y)$. Let $m^{+}$ be the larger of the two ordinals $c p(x), c p(y)$ and let $m^{-}$be the smaller. We shall also assume that among all these $x, y$ the pair $\left(m^{+}, m^{-}\right)$is minimal with respect to the lexicographical order. This means, that $\psi_{\lambda}\left(x^{\prime}+y^{\prime}\right)=\psi_{\lambda}\left(x^{\prime}\right)+\psi_{\lambda}\left(y^{\prime}\right)$ for all $x^{\prime}, y^{\prime} \in D$ with $x^{\prime} \unlhd x, y^{\prime} \unlhd y$ whereby $x^{\prime} \triangleleft x$ or $y^{\prime} \triangleleft y$. Since $\psi_{\lambda}(x), \psi_{\lambda}(y)$, and $\psi_{\lambda}(x+y)$ are continuous we conclude $\left(\psi_{\lambda}(x)\right)\left(x_{g}\right)+\left(\psi_{\lambda}(y)\right)\left(x_{q}\right) \neq\left(\psi_{\lambda}(x+y)\right)\left(x_{g}\right)$ for at least one $g \in G$. Because of [DGH, Proposition 4.8] and $\left(\psi_{\lambda}\left(x x_{g}\right)\right)\left(x_{e}\right)+\left(\psi_{\lambda}\left(y x_{g}\right)\right)\left(x_{e}\right) \neq\left(\psi_{\lambda}\left(x x_{g}+y x_{g}\right)\right)\left(x_{e}\right)$ we assume $g=e$ and discuss the three cases A1, A2.1, and A2.2 as presented in the remarks about left representations of sums and products after Definition 6.3. Using the corresponding notation and assumptions we make the following observations:

A1 : Then $\left(\psi_{\lambda}\left(x_{h}^{-1}(x+y)\right)\right)\left(x_{e}\right)=\left(\psi_{\lambda}\left(x_{h}^{-1} x\right)\right)\left(x_{e}\right)+\left(\psi_{\lambda}\left(x_{h}^{-1} y x_{g}^{-1}\right) x_{g}\right)\left(x_{e}\right)$ and therefore $\left(\psi_{\lambda}(x+y)\right)\left(x_{e}\right)=\left(\psi_{\lambda}(x)\right)\left(x_{e}\right)+\left(\psi_{\lambda}(y)\right)\left(x_{e}\right)$.

A2.1: Then $\left(\psi_{\lambda}\left(x_{h}^{-1}(x+y)\right)\right)\left(x_{e}\right)=\sum_{g \in \mathfrak{C}}\left(\psi_{\lambda}\left(d_{g}+d_{g}^{\prime}\right) x_{g}\right)\left(x_{e}\right)$. Because of $d_{g} \triangleleft x$ and $d_{g}^{\prime} \unlhd y$ we now conclude $\psi_{\lambda}\left(d_{g}+d_{g}^{\prime}\right) x_{g}=\psi_{\lambda}\left(d_{g}\right) x_{g}+\psi_{\lambda}\left(d_{g}^{\prime}\right) x_{g}$. According to 
$\left(\psi_{\lambda}\left(x_{h}^{-1} x\right)\right)\left(x_{e}\right)=\sum_{g \in \mathfrak{C}}\left(\psi_{\lambda}\left(d_{g}\right) x_{g}\right)\left(x_{e}\right)$ and $\left(\psi_{\lambda}\left(x_{h}^{-1} y\right)\right)\left(x_{e}\right)=\sum_{g \in \mathfrak{C}}\left(\psi_{\lambda}\left(d_{g}^{\prime}\right) x_{g}\right)\left(x_{e}\right)$ this means $\left(\psi_{\lambda}\left(x_{h}^{-1}(x+y)\right)\right)\left(x_{e}\right)=\left(\psi_{\lambda}\left(x_{h}^{-1} x\right)\right)\left(x_{e}\right)+\left(\psi_{\lambda}\left(x_{h}^{-1} y\right)\right)\left(x_{e}\right)$ and therefore $\left(\psi_{\lambda}(x+y)\right)\left(x_{e}\right)=\left(\psi_{\lambda}(x)\right)\left(x_{e}\right)+\left(\psi_{\lambda}(y)\right)\left(x_{e}\right)$.

A2.2 : Then $\left(\psi_{\lambda}\left(x_{h}^{-1}(x+y)\right)\right)\left(x_{e}\right)=\left(\psi_{\lambda}\left(d_{g}+d_{g}^{\prime}\right) x_{g}\right)\left(x_{e}\right)$. For all $g^{\prime} \in \mathfrak{C}, g^{\prime} \neq g$ we have $d_{g^{\prime}}+d_{g^{\prime}}^{\prime}=0$, that is $\psi_{\lambda}\left(d_{g^{\prime}}\right) x_{g^{\prime}}+\psi_{\lambda}\left(d_{g^{\prime}}^{\prime}\right) x_{g^{\prime}}=0$ and we can proceed as in case A2.1.

In any case we deduce a contradiction and we turn to statement (4). Clearly, Proposition 4.3 yields $\lambda^{\prime} \leq \lambda$ which implies $D_{<\lambda^{\prime}} \subseteq D_{<\lambda} \subseteq D_{\leq \lambda}$ where $D_{<\lambda^{\prime}}$ is a subring of $D_{\leq \lambda}$ by [DGH, Proposition 4.1]. Statement (3) ensures that $\psi_{\lambda}$ is additive on $D_{<\lambda^{\prime}}$. In order to verify the injectivity it is therefore enough to prove that $\psi_{\lambda}(x)$ is not the zero map for any non-zero $x \in D_{<\lambda^{\prime}}$. But this follows from statement (5) by the induction hypothesis since $x \in D_{\leq \lambda^{*}}$ for some $\lambda^{*}<\lambda^{\prime} \leq \lambda$ by Proposition 4.3. It remains to show the multiplicativity. The main argument we shall apply is of crucial importance and later on it will be used again several times. The idea is based on Proposition 4.3 which states that for a finite number of elements $a_{1}, \ldots, a_{n} \in D_{<\lambda^{\prime}}$ any finite number of sums of products of them belongs to a common $D_{\leq \lambda^{*}}$ where $\lambda^{*}<\lambda^{\prime} \leq \lambda$. Hence, for all these terms the corresponding images under $\psi_{\lambda}$ are already defined and the induction hypothesis can be applied. Taking into account all this we are now going to prove:

$\mathbf{M}: \psi_{\lambda}(x y)=\psi_{\lambda}(x) \psi_{\lambda}(y)$ for all $x, y \in D_{<\lambda}$.

We assume that there exist $x, y \in D_{<\lambda}$ such that $\psi_{\lambda}(x y) \neq \psi_{\lambda}(x) \psi_{\lambda}(y)$. Then $c p(x), c p(y)>1$. Similar to the proof of statement $\mathbf{A}$ we can also assume that $x, y$ are minimal in the following sense: If $x^{\prime}, y^{\prime} \in D$ such that $x^{\prime} \unlhd x, y^{\prime} \unlhd y$ whereby $x^{\prime} \triangleleft x$ or $y^{\prime} \triangleleft y$ then $\psi_{\lambda}\left(x^{\prime} y^{\prime}\right)=\psi_{\lambda}\left(x^{\prime}\right) \psi_{\lambda}\left(y^{\prime}\right)$. In addition, $\left(\psi_{\lambda}(x y)\right)\left(x_{e}\right) \neq\left(\psi_{\lambda}(x) \psi_{\lambda}(y)\right)\left(x_{e}\right)$ can be supposed since the composition of continuous endomorphisms is continuous. Moreover, let $x, y$ be reduced. Otherwise there exist $h, \bar{h} \in G$ such that $x_{\bar{h}}^{-1} y, x_{h}^{-1} x x_{\bar{h}}$ are reduced where $\left(\psi_{\lambda}\left(\left(x_{h}^{-1} x x_{\bar{h}}\right)\left(x_{\bar{h}}^{-1} y\right)\right)\right)\left(x_{e}\right) \neq\left(\psi_{\lambda}\left(x_{h}^{-1} x_{\bar{h}}\right) \psi_{\lambda}\left(x_{\bar{h}}^{-1} y\right)\right)\left(x_{e}\right)$ by statement (7). Since $x_{h}^{-1} x x_{\bar{h}}$ and $x_{\bar{h}}^{-1} y$ have the same complexity as $x$ and $y$ respectively they are minimal in the same sense as $x$ and $y$. We discuss the three cases M1,M2, and M3 as presented in the remarks about left representations of sums and products after Definition 6.3 using the corresponding notation and assumptions.

M1: Then $\left(\psi_{\lambda}(x y)\right)\left(x_{e}\right)=\sum_{g \in \mathfrak{C}}\left(\psi_{\lambda}\left(x d_{g}\right) x_{g}\right)\left(x_{e}\right)$ where $\psi_{\lambda}\left(x d_{g}\right) x_{g}=\psi_{\lambda}(x) \psi_{\lambda}\left(d_{g}\right) x_{g}$ for all $g \in \mathfrak{C}$ since $x \unlhd x$ and $d_{g} \triangleleft y$. Now, the continuity of $\psi_{\lambda}(x)$ yields

$$
\begin{aligned}
\left(\psi_{\lambda}(x) \psi_{\lambda}(y)\right)\left(x_{e}\right) & =\left(\psi_{\lambda}(x)\right)\left(\left(\psi_{\lambda}(y)\right)\left(x_{e}\right)\right)=\left(\psi_{\lambda}(x)\right)\left(\sum_{g \in \mathfrak{C}}\left(\psi_{\lambda}\left(d_{g}\right) x_{g}\right)\left(x_{e}\right)\right) \\
& =\sum_{g \in \mathfrak{C}}\left(\psi_{\lambda}(x)\right)\left(\left(\psi_{\lambda}\left(d_{g}\right) x_{g}\right)\left(x_{e}\right)\right)=\sum_{g \in \mathfrak{C}}\left(\psi_{\lambda}(x) \psi_{\lambda}\left(d_{g}\right) x_{g}\right)\left(x_{e}\right) \\
& =\sum_{g \in \mathfrak{C}}\left(\psi_{\lambda}\left(x d_{g}\right) x_{g}\right)\left(x_{e}\right)=\left(\psi_{\lambda}(x y)\right)\left(x_{e}\right) .
\end{aligned}
$$

M2: Then $\left(\psi_{\lambda}(x y)\right)\left(x_{e}\right)=\sum_{g \in \mathfrak{C}}\left(\psi_{\lambda}\left(d_{g} x_{g} y x_{g}^{-1}\right) x_{g}\right)\left(x_{e}\right)$. Because of $d_{g} \triangleleft x$ and $y \unlhd y$ we obtain $\psi_{\lambda}\left(d_{g} x_{g} y x_{g}^{-1}\right) x_{g}=\psi_{\lambda}\left(d_{g}\right) x_{g} \psi_{\lambda}(y)$ for all $g \in \mathfrak{C}$. Moreover, $y \in D_{C}^{-}$due to the specific situation of case $\mathbf{M} 2$ and therefore $\left(\psi_{\lambda}(y)\right)\left(x_{e}\right) \in F((C))$ by statement (6). 
Finally, statement (8) of Lemma 8.5 provides

$$
\begin{aligned}
\left(\psi_{\lambda}(x) \psi_{\lambda}(y)\right)\left(x_{e}\right) & =\left(\psi_{\lambda}(x)\right)\left(\left(\psi_{\lambda}(y)\right)\left(x_{e}\right)\right)=\sum_{g \in \mathfrak{C}}\left(\psi_{\lambda}\left(d_{g}\right) x_{g}\right)\left(\left(\psi_{\lambda}(y)\right)\left(x_{e}\right)\right) \\
& =\sum_{g \in \mathfrak{C}}\left(\psi_{\lambda}\left(d_{g}\right) x_{g} \psi_{\lambda}(y)\right)\left(x_{e}\right)=\sum_{g \in \mathfrak{C}}\left(\psi_{\lambda}\left(d_{g} x_{g} y x_{g}^{-1}\right) x_{g}\right)\left(x_{e}\right) \\
& =\left(\psi_{\lambda}(x y)\right)\left(x_{e}\right) .
\end{aligned}
$$

M3: Then $\left(\psi_{\lambda}(x y)\right)\left(x_{e}\right)=\sum_{g \in \mathfrak{C}}\left(\psi_{\lambda}\left(d_{g}\right) x_{g}\right)\left(x_{e}\right)$ with

$$
d_{g}=\sum_{\substack{\bar{g}, \tilde{g} \in \mathfrak{C} \\ \bar{g} \tilde{g} C^{\prime}=g C^{\prime}}} \bar{d}_{\bar{g}}\left(\left(x_{\bar{g}} \tilde{d}_{\tilde{g}} x_{\bar{g}}^{-1}\right) x_{\bar{g}} x_{\tilde{g}} x_{g}^{-1}\right)=\sum_{\substack{\bar{g}, \tilde{g} \in \mathfrak{C} \\ \bar{g} \tilde{g} C^{\prime}=g C^{\prime}}} \bar{d}_{\bar{g}} x_{\bar{g}} \tilde{d}_{\tilde{g}} x_{\tilde{g}} x_{g}^{-1}
$$

where $\bar{d}_{\bar{g}} \triangleleft x$ and $x_{\bar{g}} \tilde{d}_{\tilde{g}} x_{\tilde{g}} x_{g}^{-1} \triangleleft y$ for all $\bar{g}, \tilde{g} \in \mathfrak{C}$. Because of statement (3) and the minimality property of $x, y$ regarding their complexities we conclude by arguments similar to those above that

$$
\psi_{\lambda}\left(d_{g}\right)=\sum_{\substack{\bar{g}, \tilde{g} \in \mathfrak{C} \\ \bar{g} \tilde{g} C^{\prime}=g C^{\prime}}} \psi_{\lambda}\left(\bar{d}_{\bar{g}}\right) x_{\bar{g}} \psi_{\lambda}\left(\tilde{d}_{\tilde{g}}\right) x_{\tilde{g}} x_{g}^{-1}
$$

for any $g \in \mathfrak{C}$ where all sums are finite. We now proceed as in case M2 and apply for instance statement (8) of Lemma 8.5 since $\left(\psi_{\lambda}(y)\right)\left(x_{e}\right) \in F((C))$ by statement (6):

$$
\begin{aligned}
\left(\psi_{\lambda}(x) \psi_{\lambda}(y)\right)\left(x_{e}\right) & =\psi_{\lambda}(x)\left(\left(\psi_{\lambda}(y)\right)\left(x_{e}\right)\right) \\
& =\sum_{\bar{g} \in \mathfrak{C}}\left(\psi_{\lambda}\left(\bar{d}_{\bar{g}}\right) x_{\bar{g}}\right)\left(\left(\psi_{\lambda}(y)\right)\left(x_{e}\right)\right) \\
& =\sum_{\bar{g} \in \mathfrak{C}}\left(\psi_{\lambda}\left(\bar{d}_{\bar{g}}\right) x_{\bar{g}}\right)\left(\sum_{\tilde{g} \in \mathfrak{C}}\left(\psi_{\lambda}\left(\tilde{d}_{\tilde{g}}\right) x_{\tilde{g}}\right)\left(x_{e}\right)\right) \\
& =\sum_{\bar{g} \in \mathfrak{C}} \sum_{\tilde{g} \in \mathfrak{C}}\left(\psi_{\lambda}\left(\bar{d}_{\bar{g}}\right) x_{\bar{g}}\right)\left(\left(\psi_{\lambda}\left(\tilde{d}_{\tilde{g}}\right) x_{\tilde{g}}\right)\left(x_{e}\right)\right) \\
& =\sum_{\bar{g} \in \mathfrak{C}} \sum_{\tilde{g} \in \mathfrak{C}}\left(\psi_{\lambda}\left(\bar{d}_{\bar{g}}\right) x_{\bar{g}} \psi_{\lambda}\left(\tilde{d}_{\tilde{g}}\right) x_{\tilde{g}}\right)\left(x_{e}\right) .
\end{aligned}
$$

For a fixed $\bar{g} \in \mathfrak{C}$ the sets $\operatorname{supp}\left(\psi_{\lambda}\left(\bar{d}_{\bar{g}}\right) x_{\bar{g}} \psi_{\lambda}\left(\tilde{d}_{\tilde{g}}\right) x_{\tilde{g}}\right)\left(x_{e}\right), \tilde{g} \in \mathfrak{C}$ are pairwise disjoint since $\left(\psi_{\lambda}\left(\bar{d}_{\bar{g}}\right) x_{\bar{g}} \psi_{\lambda}\left(\tilde{d}_{\tilde{g}}\right) x_{\tilde{g}}\right)\left(x_{e}\right) \in x_{\bar{g}} x_{\tilde{g}} F\left(\left(C^{\prime}\right)\right)$ by statement $(6)$ and the induction hypothesis. Thus, Lemma 7.5 can be applied where $I=\left\{\bar{g} \in \mathfrak{C} \mid \bar{d}_{\bar{g}} \neq \mathcal{O}\right\}, J=\left\{\tilde{g} \in \mathfrak{C} \mid \tilde{d}_{\tilde{g}} \neq \mathcal{O}\right\}$, and $K=\mathfrak{C}$ with $M_{g}=\left\{(\bar{g}, \tilde{g}) \in I \times J \mid \bar{g} \tilde{g} C^{\prime}=g C^{\prime}\right\}$ for all $g \in \mathfrak{C}$. We conclude

$$
\begin{aligned}
\sum_{\bar{g} \in \mathfrak{C}} \sum_{\tilde{g} \in \mathfrak{C}}\left(\psi_{\lambda}\left(\bar{d}_{\bar{g}}\right) x_{\bar{g}} \psi_{\lambda}\left(\tilde{d}_{\tilde{g}}\right) x_{\tilde{g}}\right)\left(x_{e}\right) & =\sum_{g \in \mathfrak{C}} \sum_{(\bar{g}, \tilde{g}) \in M_{g}}\left(\psi_{\lambda}\left(\bar{d}_{\bar{g}}\right) x_{\bar{g}} \psi_{\lambda}\left(\tilde{d}_{\tilde{g}}\right) x_{\tilde{g}}\right)\left(x_{e}\right) \\
& =\sum_{g \in \mathfrak{C}}\left(\psi_{\lambda}\left(d_{g}\right) x_{g}\right)\left(x_{e}\right)=\left(\psi_{\lambda}(x y)\right)\left(x_{e}\right) .
\end{aligned}
$$

In any case we deduce a contradiction which finally shows statement $\mathbf{M}$. So far, $\psi_{\lambda}(x)$ has been defined only if $x$ belongs to some $D_{\leq \lambda^{\prime}}$ where $\lambda^{\prime}<\lambda$. Now we treat the general situation and proceed by transfinite induction on the complexity $c p(x)$ using that $\psi_{\lambda}(x)$ has already been defined if $x \in F[G, \eta, \alpha]$ or $x \in D_{\leq \lambda^{\prime}}$ for some $\lambda^{\prime}<\lambda$. 
Case 1: $x$ is a proper atom. Then $x^{-1} \triangleleft x$ and $x^{-1} \in D_{\leq \lambda^{\prime}}$ for some $\lambda^{\prime} \in \Lambda, \lambda^{\prime}<\lambda$ by [DGH, Proposition 4.1] and Proposition 4.3. According to the induction hypothesis it can be assumed that $\psi_{\lambda}\left(x^{-1}\right)$ is a continuous and $v$-compatible automorphism of $F((G))$. We define $\psi_{\lambda}(x)=\left[\psi_{\lambda}\left(x^{-1}\right)\right]^{-1}$ such that statement (5) follows by Proposition 7.3 and Theorem 7.8 . To prove statement (7) let $\tilde{g} \in G$. Then, $x_{\tilde{g}} x$ is a proper atom, $x_{\tilde{g}} x \in D_{\leq \lambda}$, and $\left.\psi_{\lambda}\left(x_{\tilde{g}} x\right)=\left[\psi_{\lambda}\left(\left(x_{\tilde{g}} x\right)^{-1}\right)\right]^{-1}=\left[\psi_{\lambda}\left(x^{-1} x_{\tilde{g}}^{-1}\right)\right]^{-1}=\left[\psi_{\lambda}\left(x^{-1}\right) x_{\tilde{g}}^{-1}\right)\right]^{-1}=$ $x_{\tilde{g}}\left[\psi_{\lambda}\left(x^{-1}\right)\right]^{-1}=x_{\tilde{g}} \psi_{\lambda}(x)$. Clearly, $\psi_{\lambda}\left(x x_{\tilde{g}}\right)=\psi_{\lambda}(x) x_{\tilde{g}}$ can be derived similarly.

We turn to the proof of statement (6). Let $x$ be reduced with pure left representation $x=\sum_{g \in \mathfrak{C}} d_{g} x_{g}$ in $D_{C}^{-}\left(\left(C / C^{\prime}, \eta, \alpha\right)\right)$ where $d_{g} \neq 0$ for at least two $g \in \mathfrak{C}$. Then $x^{-1}=\sum_{g \in \mathfrak{C}} \bar{d}_{g} x_{g}$ in $D_{C}^{-}\left(\left(C / C^{\prime}, \eta, \alpha\right)\right)$ with $\bar{d}_{g} \neq 0$ for at least two $g \in \mathfrak{C}$. This shows that $x^{-1}$ is reduced and that $x^{-1}=\sum_{g \in \mathfrak{C}} \bar{d}_{g} x_{g}$ is the pure left representation of $x^{-1}$. The sets $\operatorname{supp}\left(\psi_{\lambda}\left(d_{g}\right) x_{g}\right)\left(x_{e}\right)$ with $g \in \mathfrak{C}$ are pairwise disjoint since $\operatorname{supp}\left(\psi_{\lambda}\left(d_{g}\right) x_{g}\right)\left(x_{e}\right) \subseteq$ $x_{g} F\left(\left(C^{\prime}\right)\right)$ by induction hypothesis such that $\left\{\left(\psi_{\lambda}\left(d_{g}\right) x_{g}\right)\left(x_{e}\right) \mid g \in \mathfrak{C}\right\}$ is summable. We define $m:=\sum_{g \in \mathfrak{C}}\left(\psi_{\lambda}\left(d_{g}\right) x_{g}\right)\left(x_{e}\right) \in F((C))$. The arguments we shall apply now are essentially the same we already used before in subcase M3 of the proof of statement M. Hence, it will not be necessary to repeat all details again. We will use that $x^{-1}$ and each $d_{g}, \bar{d}_{g}$ belong to $D_{<\lambda^{\prime}}$ with $\lambda^{\prime}=c p(x)$ and that the restriction of $\psi_{\lambda}$ to $D_{<\lambda^{\prime}}$ is an injective ring homomorphism. Moreover, Proposition 4.3 provides $x^{-1} \in D_{\leq \lambda^{*}}$ for some $\lambda^{*} \in \Lambda$ with $\lambda^{*}<\lambda$ such that statements (5)-(8) of Lemma 8.5 can be applied analogously to $x^{-1}$. We obtain

$$
\begin{aligned}
\left(\psi_{\lambda}\left(x^{-1}\right)\right)(m) & =\sum_{\bar{g} \in \mathfrak{C}}\left(\psi_{\lambda}\left(\bar{d}_{\bar{g}}\right) x_{\bar{g}}\right)(m)=\sum_{\bar{g} \in \mathfrak{C}}\left(\psi_{\lambda}\left(\bar{d}_{\bar{g}}\right) x_{\bar{g}}\right)\left(\sum_{g \in \mathfrak{C}}\left(\psi_{\lambda}\left(d_{g}\right) x_{g}\right)\left(x_{e}\right)\right) \\
& =\sum_{\bar{g} \in \mathfrak{C}} \sum_{g \in \mathfrak{C}}\left(\psi_{\lambda}\left(\bar{d}_{\bar{g}} x_{\bar{g}} d_{g} x_{g}\right)\right)\left(x_{e}\right)=\sum_{\tilde{g} \in \mathfrak{C}} \sum_{\substack{\bar{g}, g \in \mathfrak{C} \\
\bar{g} g C^{\prime}=\tilde{g} C^{\prime}}}\left(\psi_{\lambda}\left(\bar{d}_{\bar{g}} x_{\bar{g}} d_{g} x_{g}\right)\right)\left(x_{e}\right) \\
& =\sum_{\tilde{g} \in \mathfrak{C}}\left(\psi_{\lambda}\left(\sum_{\substack{\bar{g}, g \in \mathfrak{C} \\
\bar{g} g C^{\prime}=\tilde{g} C^{\prime}}} \bar{d}_{\bar{g}} x_{\bar{g}} d_{g} x_{g} x_{\tilde{g}}^{-1}\right) x_{\tilde{g}}\right)\left(x_{e}\right)=\sum_{\tilde{g} \in \mathfrak{C}}\left(\psi_{\lambda}\left(\tilde{d}_{\tilde{g}}\right) x_{\tilde{g}}\right)\left(x_{e}\right)
\end{aligned}
$$

where

$$
\tilde{d}_{\tilde{g}}=\sum_{\substack{\bar{g}, g \in \mathfrak{C} \\ \bar{g} g C^{\prime}=\tilde{g} C^{\prime}}} \bar{d}_{\bar{g}} x_{\bar{g}} d_{g} x_{g} x_{\tilde{g}}^{-1} \text { for all } \tilde{g} \in \mathfrak{C} .
$$

On the other hand $\sum_{\bar{g} \in \mathfrak{C}} \bar{d}_{\bar{g}} x_{\bar{g}} \cdot \sum_{g \in \mathfrak{C}} d_{g} x_{g}=\sum_{\tilde{g} \in \mathfrak{C}} \tilde{d}_{\tilde{g}} x_{\tilde{g}}=1 x_{e}$ in $D_{C}^{-}\left(\left(C / C^{\prime}, \eta, \alpha\right)\right)$. Therefore, $\left(\psi_{\lambda}\left(x^{-1}\right)\right)(m)=x_{e}$, that is

$$
\left(\psi_{\lambda}(x)\right)\left(x_{e}\right)=\left(\left[\psi_{\lambda}\left(x^{-1}\right)\right]^{-1}\right)\left(x_{e}\right)=m=\sum_{g \in \mathfrak{C}}\left(\psi_{\lambda}\left(d_{g}\right) x_{g}\right)\left(x_{e}\right) \in F((C)) .
$$

Case 2: $x$ is additively indecomposable. Let $x=x_{1} \cdots \cdots x_{n}$ be a complete multiplicative decomposition of $x$ which implies that any $x_{i}$ is a proper atom by [DGH, Theorem 4.6]. We define $\psi_{\lambda}(x)$ and prove statements (5),(6), and (7) by induction on $n$. The case $n=1$ has been treated above and for $n>1$ we introduce $\bar{x}:=x_{2} \cdots \cdots x_{n}$. Then, $\bar{x}$ is additively indecomposable by [DGH, Lemma 4.5] with $\bar{x}=x_{2} \cdots x_{n}$ as a complete multiplicative decomposition of $\bar{x}$ by [DGH, Theorem 4.6]. Clearly, $x_{1}, \bar{x} \triangleleft x$ yields $x_{1}, \bar{x} \in D_{\leq \lambda}$ and according to Proposition 4.3 there exists $\lambda^{\prime} \in \Lambda$ with $\lambda^{\prime}<\lambda$ such that $x_{1}, \bar{x} \in D_{\leq \lambda^{\prime}}$. Hence, $\psi_{\lambda}\left(x_{1}\right)$ and $\psi_{\lambda}(\bar{x})$ are given and we define $\psi_{\lambda}(x)=\psi_{\lambda}\left(x_{1}\right) \psi_{\lambda}(\bar{x})$. 
Later on we will show that this definition does not depend on the special decomposition $x=x_{1} \cdots x_{n}$. Clearly, statement (5) follows by Proposition 7.7 and we shall prove statement (6). Thus, let $x$ be reduced. There exist $h, \bar{h} \in G$ such that $x_{h}^{-1} \bar{x}$ and $x_{\bar{h}}^{-1} x_{1} x_{h}$ are reduced. [DGH, Proposition 4.2] ensures that $x=\left(x_{1} x_{h}\right) \cdot\left(x_{h}^{-1} x_{2}\right) \cdot x_{3} \cdots x_{n}$ is a complete multiplicative decomposition of $x$ and $x_{\bar{h}}^{-1} x=\left(x_{\bar{h}}^{-1} x_{1} x_{h}\right) \cdot\left(x_{h}^{-1} x_{2}\right) \cdot x_{3} \cdots x_{n}$ is a complete multiplicative decomposition of $x_{\bar{h}}^{-1} x$ by Proposition 4.1. We define $x^{\prime}=x_{\bar{h}}^{-1} x, x_{1}^{\prime}=x_{\bar{h}}^{-1} x_{1} x_{h}$, and $\bar{x}^{\prime}=x_{h}^{-1} \bar{x}$. Now, let $a, b \in D$ arbitrary such that $a \unlhd x_{1}^{\prime}, b \unlhd \bar{x}^{\prime}$ whereby $a \triangleleft x_{1}^{\prime}$ or $b \triangleleft \bar{x}^{\prime}$. Then, $a, b, a b \triangleleft x$ because of [DGH, Theorem 4.6] such that $\psi_{\lambda}(a), \psi_{\lambda}(b)$, and $\psi_{\lambda}(a b)$ are defined and moreover $\psi_{\lambda}(a b)=\psi_{\lambda}(a) \psi_{\lambda}(b)$. The latter statement is not obvious but it can be proved exactly the same way as $\psi_{\lambda}(x y)=\psi_{\lambda}(x) \psi_{\lambda}(y)$ in statement $\mathbf{M}$ and therefore an additional proof will be omitted.

In order to show statement (6) we need the pure left representation of $x=x_{1} \bar{x}$ which will be derived from suitable left representations of $x_{1}^{\prime}$ or $\bar{x}^{\prime}$. We proceed as in the proof of statement (4) and again we have to distinguish three cases which correspond to the three cases M1, M2, and M3 as discussed in the remarks about left representations of sums and products after Definition 6.3.

M1: Then $\bar{x}^{\prime}=\sum_{g \in \mathfrak{C}} d_{g} x_{g}$ and $x_{1}^{\prime} \in D_{C}^{-}$such that $x_{\bar{h}}^{-1} x=x^{\prime}=\sum_{g \in \mathfrak{C}}\left(x_{1}^{\prime} d_{g}\right) x_{g}$ is a left representation of $x$ and $x^{\prime}$ where $\bar{h} \in C$ since $x$ is reduced. For any $g \in \mathfrak{C}$ there exists some $g^{\prime} \in \mathfrak{C}^{\mathfrak{c}}$ satisfying $\bar{h} g C^{\prime}=g^{\prime} C^{\prime}$, that is $x_{\bar{h}} x_{g} x_{g^{\prime}}^{-1} \in F^{\times} X_{C^{\prime}}$. This yields $x=\sum_{g \in \mathfrak{C}}\left(\left(x_{\bar{h}} x_{1}^{\prime} d_{g} x_{\bar{h}}^{-1}\right)\left(x_{\bar{h}} x_{g} x_{g^{\prime}}^{-1}\right)\right) x_{g^{\prime}}$ as the pure left representation of $x$. Applying the induction hypothesis to $\bar{x}^{\prime}$ and Lemma 8.5 we obtain by means of $d_{g} \triangleleft \bar{x}^{\prime}$ for all $g \in \mathfrak{C}:$

$$
\begin{aligned}
\left(\psi_{\lambda}(x)\right)\left(x_{e}\right) & =\left(\psi_{\lambda}\left(x_{1}\right)\right)\left(\left(\psi_{\lambda}(\bar{x})\right)\left(x_{e}\right)\right) \\
& =\left(\psi_{\lambda}\left(x_{\bar{h}} x_{1}^{\prime} x_{h}^{-1}\right)\right)\left(\left(\psi_{\lambda}\left(x_{h} \bar{x}^{\prime}\right)\right)\left(x_{e}\right)\right) \\
& =x_{\bar{h}}\left(\psi_{\lambda}\left(x_{1}^{\prime}\right)\right)\left(\left(\psi_{\lambda}\left(\bar{x}^{\prime}\right)\right)\left(x_{e}\right)\right) \\
& =x_{\bar{h}}\left(\psi_{\lambda}\left(x_{1}^{\prime}\right)\left(\sum_{g \in \mathfrak{C}}\left(\psi_{\lambda}\left(d_{g}\right) x_{g}\right)\left(x_{e}\right)\right)\right. \\
& =x_{\bar{h}} \sum_{g \in \mathfrak{C}}\left(\psi_{\lambda}\left(x_{1}^{\prime}\right) \psi_{\lambda}\left(d_{g}\right) x_{g}\right)\left(x_{e}\right) \\
& =x_{\bar{h}} \sum_{g \in \mathfrak{C}}\left(\psi_{\lambda}\left(x_{1}^{\prime} d_{g}\right) x_{g}\right)\left(x_{e}\right) \\
& =\sum_{g \in \mathfrak{C}}\left(\psi_{\lambda}\left(\left(x_{\bar{h}} x_{1}^{\prime} d_{g} x_{\bar{h}}^{-1}\right)\left(x_{\bar{h}} x_{g} x_{g^{\prime}}^{-1}\right)\right) x_{g^{\prime}}\right)\left(x_{e}\right) \in F((C)) .
\end{aligned}
$$

Thus, statement (6) is shown.

M2: Then $x_{1}^{\prime}=\sum_{g \in \mathfrak{C}} d_{g} x_{g}$ and $\bar{x}^{\prime} \in D_{C}^{-}$such that $x_{\bar{h}}^{-1} x=x^{\prime}=\sum_{g \in \mathfrak{C}}\left(d_{g} x_{g} \bar{x}^{\prime} x_{g}^{-1}\right) x_{g}$ is a left representation of $x$ and $x^{\prime}$. Again, $\bar{h} \in C$ since $x$ is reduced. The same arguments we just applied now show that $x=\sum_{g \in \mathfrak{C}}\left(\left(x_{\bar{h}} d_{g} x_{g} \bar{x}^{\prime} x_{g}^{-1} x_{\bar{h}}^{-1}\right)\left(x_{\bar{h}} x_{g} x_{g^{\prime}}^{-1}\right)\right) x_{g^{\prime}}$ is the pure left representation of $x$ where $x_{\bar{h}} x_{g} x_{g^{\prime}}^{-1} \in F^{\times} X_{C^{\prime}}$ for all $g \in \mathfrak{C}$. Arguments 
we repeatedly used before finally provide

$$
\begin{aligned}
\left(\psi_{\lambda}(x)\right)\left(x_{e}\right) & =\left(\psi_{\lambda}\left(x_{1}\right)\right)\left(\left(\psi_{\lambda}(\bar{x})\right)\left(x_{e}\right)\right)=x_{\bar{h}}\left(\psi_{\lambda}\left(x_{1}^{\prime}\right)\right)\left(\left(\psi_{\lambda}\left(\bar{x}^{\prime}\right)\right)\left(x_{e}\right)\right) \\
& =x_{\bar{h}} \sum_{g \in \mathfrak{C}}\left(\psi_{\lambda}\left(d_{g}\right) x_{g}\right)\left(\left(\psi_{\lambda}\left(\bar{x}^{\prime}\right)\right)\left(x_{e}\right)\right)=x_{\bar{h}} \sum_{g \in \mathfrak{C}}\left(\psi_{\lambda}\left(d_{g} x_{g} \bar{x}^{\prime} x_{g}^{-1}\right) x_{g}\right)\left(x_{e}\right) \\
& =\sum_{g \in \mathfrak{C}}\left(\psi_{\lambda}\left(\left(x_{\bar{h}} d_{g} x_{g} \bar{x}^{\prime} x_{g}^{-1} x_{\bar{h}}^{-1}\right)\left(x_{\bar{h}} x_{g} x_{g^{\prime}}^{-1}\right)\right) x_{g^{\prime}}\right)\left(x_{e}\right) \in F((C))
\end{aligned}
$$

and therefore statement (6).

M3: Then, $x_{1}^{\prime}=\sum_{\bar{g} \in \mathfrak{C}} \bar{d}_{\bar{g}} x_{\bar{g}}$ and $\bar{x}^{\prime}=\sum_{\tilde{g} \in \mathfrak{C}} \tilde{d}_{\tilde{g}} x_{\tilde{g}}$ are the pure left representations of $x_{1}^{\prime}$ and $\bar{x}^{\prime}$ respectively such that $x_{\bar{h}}^{-1} x=x^{\prime}=\sum_{g \in \mathfrak{C}} d_{g} x_{g}$ is a left representation of $x$ and $x^{\prime}$ where $\bar{h} \in C$ since $x$ is reduced and

$$
d_{g}=\sum_{\substack{\bar{g}, \tilde{g} \in \mathfrak{C} \\ \bar{g} \tilde{g} C^{\prime}=g C^{\prime}}} \bar{d}_{\bar{g}} x_{\bar{g}} \tilde{d}_{\tilde{g}} x_{\tilde{g}} x_{g}^{-1} \text { for all } g \in \mathfrak{C} .
$$

Therefore, $x=\sum_{g \in \mathfrak{C}}\left(\left(x_{\bar{h}} d_{g} x_{\bar{h}}^{-1}\right)\left(x_{\bar{h}} x_{g} x_{g^{\prime}}^{-1}\right)\right) x_{g^{\prime}}$ is the pure left representation of $x$ where $g^{\prime} \in \mathfrak{C}$ and $h g C^{\prime}=g^{\prime} C^{\prime}$ for all $g \in \mathfrak{C}$. We obtain

$$
\begin{aligned}
\left(\psi_{\lambda}(x)\right)\left(x_{e}\right) & =\left(\psi_{\lambda}\left(x_{1}\right)\right)\left(\left(\psi_{\lambda}(\bar{x})\right)\left(x_{e}\right)\right) \\
& =x_{\bar{h}}\left(\psi_{\lambda}\left(x_{1}^{\prime}\right)\right)\left(\left(\psi_{\lambda}\left(\bar{x}^{\prime}\right)\right)\left(x_{e}\right)\right) \\
& =x_{\bar{h}} \sum_{\bar{g} \in \mathfrak{C}}\left(\psi_{\lambda}\left(\bar{d}_{\bar{g}}\right) x_{\bar{g}}\right)\left(\left(\psi_{\lambda}\left(\bar{x}^{\prime}\right)\right)\left(x_{e}\right)\right) \\
& =x_{\bar{h}} \sum_{\bar{g} \in \mathfrak{C}} \sum_{\tilde{g} \in \mathfrak{C}}\left(\psi_{\lambda}\left(\bar{d}_{\bar{g}}\right) x_{\bar{g}}\right)\left(\left(\psi_{\lambda}\left(\tilde{d}_{\tilde{g}}\right) x_{\tilde{g}}\right)\left(x_{e}\right)\right) \\
& =x_{\bar{h}} \sum_{\bar{g} \in \mathfrak{C}} \sum_{\tilde{g} \in \mathfrak{C}}\left(\psi_{\lambda}\left(\bar{d}_{\bar{g}} x_{\bar{g}} \tilde{d}_{\tilde{g}} x_{\tilde{g}}\right)\right)\left(x_{e}\right) \\
& =x_{\bar{h}} \sum_{g \in \mathfrak{C}} \sum_{\bar{g} \tilde{g}, \tilde{g} \in \mathfrak{C} C^{\prime}=g C^{\prime}}\left(\psi_{\lambda}\left(\bar{d}_{\bar{g}} x_{\bar{g}} \tilde{d}_{\tilde{g}} x_{\tilde{g}} x_{g}^{-1}\right) x_{g}\right)\left(x_{e}\right) \\
& =x_{\bar{h}} \sum_{g \in \mathfrak{C}}\left(\psi_{\lambda}\left(d_{g}\right) x_{g}\right)\left(x_{e}\right) \\
& =\sum_{g \in \mathfrak{C}}\left(\psi_{\lambda}\left(x_{\bar{h}} d_{g} x_{\bar{h}}^{-1} x_{\bar{h}} x_{g} x_{g^{\prime}}^{-1}\right) x_{g^{\prime}}\right)\left(x_{e}\right) \in F((C))
\end{aligned}
$$

which shows statement (6).

We still need to prove that $\psi_{\lambda}(x)$ is well-defined. Thus, let $x=x_{1} \cdot x_{2} \cdots x_{n}$, $x=y_{1} \cdot y_{2} \cdots y_{m}$ be two complete multiplicative decompositions of $x$. We define $\bar{x}=x_{2} \cdots x_{n}$ and $\bar{y}=y_{2} \cdots y_{m}$. Since both endomorphisms are continuous it is enough to show $\left(\psi_{\lambda}\left(x_{1}\right) \psi_{\lambda}(\bar{x})\right)\left(x_{g}\right)=\left(\psi_{\lambda}\left(y_{1}\right) \psi_{\lambda}(\bar{y})\right)\left(x_{g}\right)$, that is, $\left(\psi_{\lambda}\left(x_{1}\right) \psi_{\lambda}\left(\bar{x} x_{g}\right)\right)\left(x_{e}\right)=$ $\left(\psi_{\lambda}\left(y_{1}\right) \psi_{\lambda}\left(\bar{y} x_{g}\right)\right)\left(x_{e}\right)$ for an arbitrary $g \in G$. According to (the remark after) Proposition 4.1 the element $x x_{g}$ is additively indecomposable with two complete additive decompositions

$$
x x_{g}=x_{1} \cdots x_{n-1} \cdot\left(x_{n} x_{g}\right)=y_{1} \cdots y_{m-1} \cdot\left(y_{m} x_{g}\right) .
$$


Without loss of generality, we may therefore suppose $g=e$. Since $x_{h}^{-1} x$ is reduced for some $h \in G$ we may also assume for the same reason that $x$ itself is reduced. But then $\left(\psi_{\lambda}(x)\right)\left(x_{e}\right)$ depends only on the pure left-representation of $x$ and not on the complete multiplicative decomposition as we have just proved. This shows, that $\psi_{\lambda}(x)$ is welldefined if $x$ is additively indecomposable and also statement (6) is shown. We finally turn to statement (7) and consider $\tilde{g} \in G$. Clearly, $x_{\tilde{g}} x=\left(x_{\tilde{g}} x_{1}\right) \cdot x_{2} \cdots x_{n}$ is a complete multiplicative decomposition of the additively indecomposable element $x_{\tilde{g}} x$ by Proposition 4.1. Hence,

$$
\begin{aligned}
\psi_{\lambda}\left(x_{\tilde{g}} x\right) & =\psi_{\lambda}\left(x_{\tilde{g}} x_{1}\right) \cdot \psi_{\lambda}\left(x_{2} \cdots x_{n}\right) \\
& =x_{\tilde{g}} \psi_{\lambda}\left(x_{1}\right) \cdot \psi_{\lambda}\left(x_{2} \cdots x_{n}\right)=x_{\tilde{g}} \psi_{\lambda}(x)
\end{aligned}
$$

and the claim for $x x_{\tilde{g}}$ follows similarly.

Case 3: Now, let $x$ be an arbitrary element having a complete additive decomposition $x=x_{1}+\cdots+x_{n}, n \in \mathbb{N}$. Then any $x_{i}, i=1, \ldots, n$ is additively indecomposable by [DGH, Theorem 3.6]. We define $\psi_{\lambda}(x)$ and prove statemants (6) and (7) by induction on $n$. The case $n=1$ has been treated above. Thus, let $n>1$ and $\bar{x}=x_{2}+\cdots+x_{n}$ which presents in addition a complete additive decomposition of $\bar{x}$ due to [DGH, Theorem 3.6]. Clearly, $x_{1}, \bar{x} \triangleleft x$ yields $x_{1}, \bar{x} \in D_{\leq \lambda}$ by Proposition 4.3 such that $\psi_{\lambda}\left(x_{1}\right), \psi_{\lambda}(\bar{x})$ are already given by induction hypothesis. We define $\psi_{\lambda}(x)=\psi_{\lambda}\left(x_{1}\right)+\psi_{\lambda}(\bar{x})$ and will prove later on that this definition does not depend on the complete additive decomposition of $x$ as introduced above. In order to show statement (6) let $x$ be reduced and let $c p\left(x_{1}\right)>1$ or $c p(\bar{x})>1$. The pure left representation of $x=x_{1}+\bar{x}$ results from left representations of $x_{1}$ or $\bar{x}$ where two different situations must be considered which correspond to the cases A1 and A2 .

A1: Without loss of generality (if necessary the roles of $x_{1}$ and $\bar{x}$ have to be exchanged) there exist $h \in G, C \in \mathcal{C}_{\leq}^{*}$, and $g \in \mathfrak{C}, g \neq e$ such that $x_{h}^{-1} x_{1}, x_{h}^{-1} \bar{x} x_{g}^{-1} \in D_{C}^{-}$and

$$
x_{h}^{-1} x=x_{h}^{-1}\left(x_{1}+\bar{x}\right)=x_{h}^{-1} x_{1}+\left(x_{h}^{-1} \bar{x} x_{g}^{-1}\right) x_{g}
$$

is a left representation of $x$. Since $x$ is reduced $h \in C$ follows and $x_{1} x_{h}^{-1}, \bar{x} x_{g}^{-1} x_{h}^{-1} \in D_{C}^{-}$. There exist $\bar{h}, \bar{g} \in \mathfrak{C}$ satisfying $h C^{\prime}=\bar{h} C^{\prime}$ and $h g C^{\prime}=\bar{g} C^{\prime}$ where $\bar{h} \neq \bar{g}$ because of $g \notin C$. Hence $x_{1} x_{\bar{h}}^{-1}=\left(x_{1} x_{h}^{-1}\right)\left(x_{h} x_{\bar{h}}^{-1}\right), \bar{x} x_{\bar{g}}^{-1}=\left(\bar{x} x_{g}^{-1} x_{h}^{-1}\right)\left(x_{h} x_{g} x_{\bar{g}}^{-1}\right) \in D_{C}^{-}$such that

$$
x=\left(x_{1} x_{\bar{h}}^{-1}\right) x_{\bar{h}}+\left(\bar{x} x_{\bar{g}}^{-1}\right) x_{\bar{g}}
$$

is the pure left representation of $x$ and statement (6) follows from

$$
\begin{aligned}
\left(\psi_{\lambda}(x)\right)\left(x_{e}\right) & =\left(\psi_{\lambda}\left(x_{1}\right)\right)\left(x_{e}\right)+\left(\psi_{\lambda}(\bar{x})\right)\left(x_{e}\right) \\
& =\left(\psi_{\lambda}\left(x_{1} x_{\bar{h}}^{-1}\right) x_{\bar{h}}\right)\left(x_{e}\right)+\left(\psi_{\lambda}\left(\bar{x} x_{\bar{g}}^{-1}\right) x_{\bar{g}}\right)\left(x_{e}\right) .
\end{aligned}
$$

A2: Then, there exist left representations $x_{h}^{-1} x_{1}=\sum_{g \in \mathfrak{C}} d_{g} x_{g}$ and $x_{h}^{-1} \bar{x}=\sum_{g \in \mathfrak{C}} d_{g}^{\prime} x_{g}$ for $x_{1}$ and $\bar{x}$ respectively. Because of $d_{g}+d_{g}^{\prime} \triangleleft x_{1}+\bar{x}=x$ the subcase A2.2 can not occur such that $x_{h}^{-1} x=x_{h}^{-1} x_{1}+x_{h}^{-1} \bar{x}=\sum_{g \in \mathfrak{C}}\left(d_{g}+d_{g}^{\prime}\right) x_{g}$ is a left representation of $x$. Since $x$ is reduced we obtain

$$
x=\sum_{g \in \mathfrak{C}}\left(\left(x_{h}\left(d_{g}+d_{g}^{\prime}\right) x_{h}^{-1}\right)\left(x_{h} x_{g} x_{g^{\prime}}^{-1}\right)\right) x_{g^{\prime}}
$$


as the pure left representation of $x$ where $g^{\prime} \in \mathfrak{C}$ and $h g C^{\prime}=g^{\prime} C^{\prime}$ for all $g \in \mathfrak{C}$. Now, statement (6) follows from

$$
\begin{aligned}
\left(\psi_{\lambda}(x)\right)\left(x_{e}\right) & =\left(\psi_{\lambda}\left(x_{1}\right)\right)\left(x_{e}\right)+\left(\psi_{\lambda}(\bar{x})\right)\left(x_{e}\right) \\
& =x_{h} \sum_{g \in \mathfrak{C}}\left[\left(\psi_{\lambda}\left(d_{g}\right) x_{g}\right)\left(x_{e}\right)+\left(\psi_{\lambda}\left(d_{g}^{\prime}\right) x_{g}\right)\left(x_{e}\right)\right] \\
& =x_{h} \sum_{g \in \mathfrak{C}}\left(\left(\psi_{\lambda}\left(d_{g}\right)+\psi_{\lambda}\left(d_{g}^{\prime}\right)\right) x_{g}\right)\left(x_{e}\right) \\
& =x_{h} \sum_{g \in \mathfrak{C}}\left(\psi_{\lambda}\left(d_{g}+d_{g}^{\prime}\right) x_{g}\right)\left(x_{e}\right) \\
& =\sum_{g \in \mathfrak{C}}\left(\psi_{\lambda}\left(\left(x_{h}\left(d_{g}+d_{g}^{\prime}\right) x_{h}^{-1}\right)\left(x_{h} x_{g} x_{g^{\prime}}^{-1}\right)\right) x_{g^{\prime}}\right)\left(x_{e}\right) .
\end{aligned}
$$

To show that $\psi_{\lambda}(x)$ is well-defined let $x=x_{1}+\cdots+x_{n}, x=y_{1}+\cdots+y_{m}$ be two complete additive decompositions of $x$ and $\bar{x}=x_{2}+\cdots+x_{n}, \bar{y}=y_{2}+\cdots+y_{m}$. We assume $\psi_{\lambda}\left(x_{1}\right)+\psi_{\lambda}(\bar{x}) \neq \psi_{\lambda}\left(y_{1}\right)+\psi_{\lambda}(\bar{y})$, that is, $\left(\psi_{\lambda}\left(x_{1}\right)+\psi_{\lambda}(\bar{x})\right)\left(x_{g}\right) \neq\left(\psi_{\lambda}\left(y_{1}\right)+\psi_{\lambda}(\bar{y})\right)\left(x_{g}\right)$ and therefore $\left(\psi_{\lambda}\left(x_{1} x_{g}\right)+\psi_{\lambda}\left(\bar{x} x_{g}\right)\right)\left(x_{e}\right) \neq\left(\psi_{\lambda}\left(y_{1} x_{g}\right)+\psi_{\lambda}\left(\bar{y} x_{g}\right)\right)\left(x_{e}\right)$ for some $g \in G$ since both endomorphisms are continuous. Furthermore, $x x_{g}=x_{1} x_{g}+\cdots+x_{n} x_{g}$, $x x_{g}=y_{1} x_{g}+\cdots+y_{m} x_{g}$ are two complete additive decompositions of $x x_{g}$ by Proposition 4.1 which means that we may restrict to the case $g=e$. Since $x_{h}^{-1} x$ is reduced for some $h \in G$ we may also assume for the same reason that $x$ itself is reduced. But then $\left(\psi_{\lambda}(x)\right)\left(x_{e}\right)$ depends only on the pure left-representation of $x$ and not on the complete additive decomposition as we have just proved. This shows, that $\psi_{\lambda}(x)$ is well-defined if $x$ is additively indecomposable and also statement (6) is shown. In order to verify statement (7) we consider an arbitrary $\tilde{g} \in G$. Again, $x_{\tilde{g}} x=x_{\tilde{g}} x_{1}+x_{\tilde{g}} x_{2}+\cdots+x_{\tilde{g}} x_{n}$ is a complete additive decomposition of $x_{\tilde{g}} x$ by Proposition 4.1 such that

$$
\begin{aligned}
\psi_{\lambda}\left(x_{\tilde{g}} x\right) & =\psi_{\lambda}\left(x_{\tilde{g}} x_{1}\right)+\psi_{\lambda}\left(x_{\tilde{g}} x_{2}+\cdots+x_{\tilde{g}} x_{n}\right) \\
& =x_{\tilde{g}} \psi_{\lambda}\left(x_{1}\right)+x_{\tilde{g}} \psi_{\lambda}\left(x_{2}+\cdots+x_{n}\right)=x_{\tilde{g}} \psi_{\lambda}(x) .
\end{aligned}
$$

Similar arguments provide the claim for $x x_{\tilde{g}}$.

It remains to prove statement (5). Since $\psi_{\lambda}(x)$ is continuous by induction hypothesis and Proposition 7.7 we apply Theorem 7.10 where $x \neq 0$ and show that $\psi_{\lambda}(x)$ is surjective on $G$ and $v$-compatible on $G$.

$\psi_{\lambda}(x)$ is $v$-compatible on $G$ : We assume that $\psi_{\lambda}(y)$ is $v$-compatible on $G$ for each $y \in D, y \neq 0$ where $y \triangleleft x$ and consider $\bar{g}, \tilde{g} \in G$ with $\bar{g}<\tilde{g}$. Then, $v\left(\left(\psi_{\lambda}(x)\right)\left(x_{\bar{g}}\right)\right)<$ $v\left(\left(\psi_{\lambda}(x)\right)\left(x_{\tilde{g}}\right)\right)$ has to be shown, that is, $v\left(\left(\psi_{\lambda}\left(x x_{\bar{g}}\right)\right)\left(x_{e}\right)\right)<v\left(\left(\psi_{\lambda}\left(x x_{\bar{g}}\right)\right)\left(x_{\bar{g}}^{-1} x_{\tilde{g}}\right)\right)=$ $v\left(\left(\psi_{\lambda}\left(x x_{\bar{g}}\right)\right)\left(x_{\bar{g}^{-1} \tilde{g}}\right)\right)$ where $e<\bar{g}^{-1} \tilde{g}$. Therefore, without loss of generality let us assume $\bar{g}=e$. Moreover, we may restrict to the case where $c p(x)>1$ and $x$ is reduced. Let $x=\sum_{g \in \mathfrak{C}} d_{g} x_{g} \in D_{C}^{-}\left(\left(C / C^{\prime}, \eta, \alpha\right)\right)$ be the pure left representation of $x$ and where $g_{0}$ is the minimal element in the support of $x$. Two cases have to be differentiated here:

Case 1: $\tilde{g} \notin C$. Then $C \subseteq C_{\tilde{g}}^{-}$and $x \in D_{C}^{+} \subseteq D_{\tilde{g}}^{-}$. This means $x_{\tilde{g}}^{-1} x x_{\tilde{g}} \in D_{\tilde{g}}^{-}$, that is, $\left(\psi_{\lambda}\left(x_{\tilde{g}}^{-1} x x_{\tilde{g}}\right)\right)\left(x_{e}\right) \in F\left(\left(C_{\tilde{g}}^{-}\right)\right)$and $\left(\psi_{\lambda}(x)\right)\left(x_{\tilde{g}}\right)=x_{\tilde{g}}\left(\psi_{\lambda}\left(x_{\tilde{g}}^{-1} x x_{\tilde{g}}\right)\right)\left(x_{e}\right) \in x_{\tilde{g}} F\left(\left(C_{\tilde{g}}^{-}\right)\right)$ follows. Because of $\left(\psi_{\lambda}(x)\right)\left(x_{e}\right) \in F((C)) \subseteq F\left(\left(C_{\tilde{g}}^{-}\right)\right)$and $\tilde{g}>e$ we are done.

Case 2: $\tilde{g} \in C$. For $g \in \mathfrak{C}$ there exists $g^{\prime} \in \mathfrak{C}$ satisfying $g \tilde{g} C^{\prime}=g^{\prime} C^{\prime}$. Since $C / C^{\prime}$ is 
abelian we conclude $g^{\prime}<\bar{g}^{\prime}$ for all $g, \bar{g} \in \mathfrak{C}$ with $g<\bar{g}$. This shows that

$$
x x_{\tilde{g}}=\sum_{g \in \mathfrak{C}}\left(d_{g}\left(x_{g} x_{\tilde{g}} x_{g^{\prime}}^{-1}\right)\right) x_{g^{\prime}}
$$

is a left representation of $x x_{\tilde{g}}$ where $g_{0}^{\prime}$ is the minimal element in the support of $x x_{\tilde{g}}$. For all $g \in \mathfrak{C}$ we obtain

$$
\left(\psi_{\lambda}\left(d_{g}\left(x_{g} x_{\tilde{g}} x_{g^{\prime}}^{-1}\right)\right) x_{g^{\prime}}\right)\left(x_{e}\right) \in x_{g^{\prime}} F\left(\left(C^{\prime}\right)\right),
$$

that is

$$
v\left(\left(\psi_{\lambda}(x)\right)\left(x_{\tilde{g}}\right)\right)=v\left(\left(\psi_{\lambda}\left(x x_{\tilde{g}}\right)\right)\left(x_{e}\right)\right)=v\left(\left(\psi_{\lambda}\left(d_{g_{0}} x_{g_{0}} x_{\tilde{g}}\right)\right)\left(x_{e}\right)\right)=v\left(\left(\psi_{\lambda}\left(d_{g_{0}} x_{g_{0}}\right)\right)\left(x_{\tilde{g}}\right)\right) .
$$

Clearly, $v\left(\left(\psi_{\lambda}(x)\right)\left(x_{e}\right)\right)=v\left(\left(\psi_{\lambda}\left(d_{g_{0}} x_{g_{0}}\right)\right)\left(x_{e}\right)\right)$ follows in a similar fashion. Because of $d_{g_{0}} \triangleleft x$ and $\tilde{g}>e$ we obtain $v\left(\left(\psi_{\lambda}\left(d_{g_{0}} x_{g_{0}}\right)\right)\left(x_{e}\right)\right)<v\left(\left(\psi_{\lambda}\left(d_{g_{0}} x_{g_{0}}\right)\right)\left(x_{\tilde{g}}\right)\right)$ as claimed.

$\psi_{\lambda}(x)$ is surjective on $G$ : We assume that $\psi_{\lambda}(y)$ is surjective on $G$ for all $y \in D, y \neq 0$ where $y \triangleleft x$ and choose an arbitrary $\tilde{g} \in G$. It has to be shown that $v\left(\left(\psi_{\lambda}(x)\right)\left(x_{\bar{g}}\right)\right)=\tilde{g}$ holds true for some $\bar{g} \in G$ which is equivalent to $v\left(\left(\psi_{\lambda}\left(x_{\tilde{g}}^{-1} x\right)\right)\left(x_{\bar{g}}\right)\right)=e$. Thus, let $\tilde{g}=e$ without loss of generality and let $x x_{h}^{-1}=\sum_{g \in \mathfrak{C}} d_{g} x_{g}$ be a right representation of $x$. If there is some $\bar{g} \in G$ satisfying $v\left(\left(\psi_{\lambda}\left(x x_{h}^{-1}\right)\right)\left(x_{\bar{g}}\right)\right)=e$ then $v\left(\left(\psi_{\lambda}(x)\right)\left(x_{h^{-1} \bar{g}}\right)\right)=e$ and vice versa. Therefore, we may choose $h=e$, that is $x=\sum_{g \in \mathfrak{C}} d_{g} x_{g}$. Because of $d_{g_{0}} \triangleleft x$ there exists some $\bar{g} \in G$ satisfying

$$
v\left(\left(\psi_{\lambda}\left(d_{g_{0}} x_{g_{0}} x_{\bar{g}}\right)\right)\left(x_{e}\right)\right)=v\left(\left(\psi_{\lambda}\left(d_{g_{0}} x_{g_{0}}\right)\right)\left(x_{\bar{g}}\right)\right)=e
$$

where $g_{0}$ is minimal in the support of $x$. If $\bar{g} \notin C$, which means $C \subseteq C_{\bar{g}}^{-}$, then $D_{C}^{+} \subseteq D_{\bar{g}}^{-}$ and $x_{\bar{g}}^{-1} d_{g_{0}} x_{g_{0}} x_{\bar{g}} \in D_{\bar{g}}^{-}$since $d_{g_{0}} x_{g_{0}} \in D_{C}^{-} \subseteq D_{\bar{g}}^{-}$. Hence, $x_{\bar{g}}\left(\psi_{\lambda}\left(x_{\bar{g}}^{-1} d_{g_{0}} x_{g_{0}} x_{\bar{g}}\right)\right)\left(x_{e}\right)$ is in $x_{\bar{g}} F\left(\left(C_{\bar{g}}^{-}\right)\right)$. But this would be contrary to $v\left(\left(\psi_{\lambda}\left(d_{g_{0}} x_{g_{0}} x_{\bar{g}}\right)\right)\left(x_{e}\right)\right)=e$. Thus, $\bar{g} \in C$ follows and again for any $g \in \mathfrak{C}$ let $g^{\prime}$ be in $\mathfrak{C}$ satisfying $g \bar{g} C^{\prime}=g^{\prime} C^{\prime}$. Similar to the arguments we used above we conclude $\left(\psi_{\lambda}(x)\right)\left(x_{\bar{g}}\right)=\sum_{g \in \mathfrak{C}}\left(\psi_{\lambda}\left(d_{g}\left(x_{g} x_{\bar{g}} x_{g^{\prime}}^{-1}\right)\right) x_{g^{\prime}}\right)\left(x_{e}\right)$ which provides

$$
v\left(\left(\psi_{\lambda}(x)\right)\left(x_{\bar{g}}\right)\right)=v\left(\left(\psi_{\lambda}\left(d_{g_{0}} x_{g_{0}}\right) x_{\bar{g}}\right)\left(x_{e}\right)\right)=v\left(\left(\psi_{\lambda}\left(d_{g_{0}} x_{g_{0}}\right)\right)\left(x_{\bar{g}}\right)\right)=e .
$$

We finally turn to the proof of Theorem 8.1. For any $x \in D$ there exists some $\lambda \in \Lambda$ satisfying $x \in D_{\leq \lambda}$ and Lemma 8.6 now shows that $\psi(x):=\psi_{\lambda}(x)$ where $\lambda \in \Lambda$ and $x \in D_{\leq \lambda}$ yields a well-defined mapping $\psi: D \longrightarrow \mathcal{R}$. In order to show that $\psi$ is additive let us consider arbitrary elements $x, y \in D$. Then $x, y \in D_{\leq \lambda}$ for some $\lambda \in \Lambda$. We have to verify $\psi_{\lambda}(x+y)=\psi_{\lambda}(x)+\psi_{\lambda}(y)$ which can be done as in the proof of statement A. Using the notation introduced there let us assume $\psi_{\lambda}(x+y) \neq \psi_{\lambda}(x)+\psi_{\lambda}(y)$ and that $\left(\mathrm{m}^{+}, \mathrm{m}^{-}\right)$is minimal with respect to the lexicographical order among all $x, y \in D_{\leq \lambda}$. Now, the arguments we applied in the proof of statement $\mathbf{A}$ can be adopted straightforwardly. Similarly, the multiplicativity of $\psi$ can be derived as statement $\mathbf{M}$. Thus, $\psi$ is a ring homomorphism which in addition is injective because of statement (5) of Lemma 8.6. Therefore, $\psi(D)$ is a subdivision ring of $\mathcal{R}$ containing $F[G, \eta, \alpha]$ as a subring. Since $\mathcal{R}$ is a division ring of fractions of $F[G, \eta, \alpha]$ we conclude that $\psi$ is surjective. 


\section{Hughes' Theorems And An Example}

We now discuss I. Hughes' two main theorems from [H1, $\mathrm{H} 2$ in the light of our results.

Theorem 9.1. Let $R=F[G, \eta, \alpha]$ be a crossed product of a locally indicable group $G$ over a skew field $F$. If $R$ has two Hughes-free division rings of fractions $D$ and $D^{\prime}$, then they are isomorphic by an isomorphism which extends the identity map on $R$.

Proofs are given in [H1, DHS, J]. According to the arguments from [J] we obtain this theorem from Corollary 8.2 and the fact that both division rings are free with respect to a Conradian left-order (with maximal rank) of $G$. Following [H2] we call a locally indicable group $G$ freely embeddable if $F[G, \eta, \alpha]$ has a Hughes-free division ring of fractions for each skew field $F$. The next theorem is the main result of [H2].

Theorem 9.2. Let $G$ be a locally indicable group and let $N$ be a normal subgroup of $G$. If $G / N$ and $N$ are both freely embeddable then $G$ is also freely embeddable.

Proof. Let $F[G, \eta, \alpha]$ be an arbitrary crossed product of $G$ over a skew field $F$. According to Proposition 3.1 we consider $F[G, \eta, \alpha]$ as a crossed product of $G / N$ over the ring $F[N, \eta, \alpha]$. Therefore, we shall write $(F[N, \eta, \alpha])[G / N, \bar{\eta}, \bar{\alpha}]$ instead of $F[G, \eta, \alpha]$ where $\bar{\alpha}_{g}$ is the restriction of $\alpha_{g}$ to $F[N, \eta, \alpha]$ for all $g \in G$ which is an automorphism of $F[N, \eta, \alpha]$. By assumption, $F[N, \eta, \alpha]$ possesses a Hughes-free division ring of fractions $D_{N}$. Because of Theorem 9.1 (or Corollary 8.2 ) any $\bar{\alpha}_{g}, g \in G$ can be extended to an automorphism of $D_{N}$. This extension is unique since $D_{N}$ is a division ring of fractions of $F[N, \eta, \alpha]$ and therefore it will also be denoted by $\bar{\alpha}_{g}$. As explained in Section 3 before Proposition 3.2 the crossed product $D_{N}[G / N, \bar{\eta}, \bar{\alpha}]$ is well-defined and contains $F[G, \eta, \alpha]$ as a subring in the obvious meaning. By assumption, $D_{N}[G / N, \bar{\eta}, \bar{\alpha}]$ possesses a Hughes-free division ring of fractions $D$. Clearly, $D$ is a division ring of fractions of $F[G, \eta, \alpha]$ and it remains to prove that it is Hughes-free. We show that $D$ is free with respect to a Conradian left-order $\leq$ of $G$. Let $\leq_{1}$ and $\leq_{2}$ be Conradian left-orders of $N$ and $G / N$ respectively. Then, $P=P_{\leq_{1}} \cup\left\{g \in G \mid e N<_{2} g N\right\}$ is a positive cone of $G$ of a suitable left-order $\leq$ of $G$. A subgroup $U$ of $G$ is convex in $G$ with respect to $\leq$ if and only if $U \subseteq N$ and $U$ is convex in $N$ with respect to $\leq_{1}$ or $N \subseteq U$ and $U / N$ is a convex subgroup of $G / N$ with respect to $\leq_{2}$. This shows that $\leq$ is Conradian. We consider an arbitrary convex jump $\left(C^{\prime}, C\right)$ of $G$ with respect to $\leq$ and arbitrarily given $h_{1}, \ldots, h_{n} \in C$ such that $h_{1} C^{\prime}, \ldots, h_{n} C^{\prime}$ are pairwise different. Then $C^{\prime} \subset C \subseteq N$ or $N \subseteq C^{\prime} \subset C$. In the first case we obtain that $\left(C^{\prime}, C\right)$ is a convex jump of $N$ and that the rational closure of $F\left[C^{\prime}, \eta, \alpha\right]$ in $D$ coincides with the rational closure $D_{C}^{-}$of $F\left[C^{\prime}, \eta, \alpha\right]$ in $D_{N}$. Since $D_{N}$ is a free division ring of fractions of $F[N, \eta, \alpha]$ with respect to $\leq_{1}$ we conclude that $x_{h_{1}}, \ldots, x_{h_{n}}$ are linearly independent over $D_{C}^{-}$. In the second case we obtain that $D_{C}^{-}$is the rational closure of $F\left[C^{\prime}, \eta, \alpha\right]$ in $D$. Then $\left(C^{\prime} / N, C / N\right)$ is a convex jump of $G / N$ with respect to $\leq_{2}$ by definition of $\leq$ and $\left(h_{1} N\right) C^{\prime} / N, \ldots,\left(h_{n} N\right) C^{\prime} / N$ are pairwise different. Moreover, $D_{C}^{-}$is the rational closure of $D_{N}\left[C^{\prime} / N, \bar{\eta}, \bar{\alpha}\right]$ in $D$. Applying the notation of Proposition 3.1 we choose $h_{1}^{\prime}, \ldots h_{n}^{\prime} \in \mathfrak{G}$ satisfying $h_{1}^{\prime} N=h_{1} N, \ldots, h_{n}^{\prime} N=h_{n} N$. Then, $x_{h_{1}^{\prime}}, \ldots, x_{h_{n}^{\prime}}$ are linearly independent over $D_{C}^{-}$since $D$ is a free division ring of fractions of $D_{N}[G / N, \bar{\eta}, \bar{\alpha}]$ with respect to $\leq_{2}$ and the same holds true for $x_{h_{1}}, \ldots, x_{h_{n}}$. 
We finally apply our results to a locally indicable group $G$ with a left-order which is not Conradian and refer the reader to [D2, D3, GS] for further details. Let $G$ be the knot group of the trefoil which is isomorphic to the braid group $B_{3}$ on 3 strands. It can be written as a semi-direct product $B_{3} \cong F_{2} \rtimes_{\varphi}\langle x\rangle$ where $F_{2}$ is the free group of rank 2 and $\langle x\rangle$ an infinite cyclic group. As a free group $F_{2}$ is endowed with a two-sided order and therefore the group ring $F\left[F_{2}\right]$ of $F_{2}$ over an arbitrary skew field $F$ possesses a Hughesfree division ring of fractions $D_{F_{2}}$ which is given, for instance, as the rational closure of $F\left[F_{2}\right]$ in the corresponding Mal'cev-Neumann division ring of all formal power series in $F_{2}$ over $F$. As explained in the proof of Theorem 9.2 the group ring $F\left[B_{3}\right]$ occurs as a subring of the skew polynomial ring $D_{F_{2}}[x, \varphi]$ which is a left and right Ore-domain. The corresponding division ring of fractions $D_{F_{2}}(x, \varphi)$ is a Hughes-free division ring of fractions of $F\left[B_{3}\right]$. On the other hand $B_{3}$ can be written as $B_{3}=\left\langle u, w \mid w u^{2} w=u\right\rangle$ where $u$ and $w$ generate a semigroup $P \subseteq B_{3}$ which is a positive cone of a left-order $\leq \prime$ of $B_{3}$ that is not Conradian. The corresponding Dubrovin-quotient $\operatorname{ring} \mathcal{R}$ of $F\left[B_{3}\right]$, that is, the rational closure of $F\left[B_{3}\right]$ in the endomorphism ring of the right $F$-vector space $F\left(\left(B_{3}\right)\right)$ of all formal power series in $B_{3}$ over $F$ with respect to $\leq^{\prime}$ is a division ring. Even though $\leq^{\prime}$ is not Conradian the division ring $\mathcal{R}$ is Hughes-free by Corollary 7.15, that is, $\mathcal{R}$ and $D_{F_{2}}(x, \varphi)$ are isomorphic. Therefore, $D_{F_{2}}(x, \varphi)$ has a rank 1 valuation ring possessing a prime ideal which is not completely prime.

\section{REFERENCES}

[Bo] A.A. Bovdi, Crossed products of a semigroup and a ring, Dokl. Akad. Nauk SSSR 137 (1961), 1267-1269, (Russian).

[Br] S. D. Brodskii, Equations over groups, and groups with one defining relation, Sibirsk. Mat. Zh. 25 (1984), no. 2, 84-103.

[BH] R. G. Burns, V. W. Hale, A note on group rings of certain torsion-free groups, Canad. Math. Bull. 15 (1972), 441-445.

[CR] A. Clay, D. Rolfsen, Ordered groups and topology, Graduate Studies in Mathematics 176, American Mathematical Society, Providence, RI, 2016.

[Co] P.M. Cohn, An Introduction to Ring Theory, Springer Undergraduate Mathematics Series, Springer, 2000.

[C] P.F. Conrad, Right-ordered groups, Mich. Math. J. 6 (3) (1959), 267-275.

[DHS] W. Dicks, D. Herbera, J. Sánchez, On a Theorem of Ian Hughes About Division Rings of Fractions, Comm. Alg. 32(3) (2004), 1127-1149.

[D1] N.I. Dubrovin, Invertibility of the group ring of a right-ordered group over a division ring, Mat. Zametki 42 (4) (1987), 508-518 (Russian); translation in Math. Notes 42 (3-4) (1982), 781-786.

[D2] N.I. Dubrovin, The rational closure of group rings of left-orderable groups, Mat. Sbornik 184 (7) (1993), 3-48 (Russian); translation in Russian Acad. Sci. Sb. Math. 79 (2) (1993), 231-263.

[D3] N.I. Dubrovin, The rational closure of group rings of left-ordered groups, SM-DU-254, Duisburg, 1994.

[D4] N.I. Dubrovin, Formal sums and power series over a group, Mat. Sbornik 191 (7) (2000), 13-30 (Russian); translation in Sb. Math. 191 (7-8)(2000), 955-971.

[DGH] N.I. Dubrovin, J. Gräter, T.Hanke, Complexity of Elements in Rings, Algebr. Represent. Theory 6(1) (2003), 33-45.

[G] J. Gräter, Left-Orderings, Power Series, Valuations, unpublished, Universität Potsdam, April 2015

[GS] J. Gräter, R.P. Sperner, On Embedding Left-Ordered Groups into Division Rings, Forum Mathematicum 27 (2015), 485-518. 
[Hö] O. Hölder, Die Axiome der Quantität und die Lehre vom Maß, Ber. Verh. Kgl. sächs. Ges. Wiss. Leipzig, math.-phys. Kl. 53 (1901), 1 - 64.

[Hi] G. Higman, The units of group rings, Proc. London Math. Soc. 46 (1940), no. 2, 231-248.

[H1] I. Hughes, Division Rings of Fractions for Group Rings, Comm. Pure Appl. Math. 23 (1970), 181-188.

[H2] I. Hughes, Division Rings of Fractions for Group Rings II, Comm. Pure Appl. Math. 25 (1972), 127-131.

$[J] \quad$ F. Jakobs, Dubrovin-Rings and Their Connection to Hughes-Free Skew Fields of Fractions, Doctoral Dissertation, Universität Potsdam, 2019.

[JL] A. Jaikin-Zapirain, D. López-Álvarez, The Strong Atiyah Conjecture for One-Relator Groups, preprint (2018), https://arxiv.org/abs/1810.12135.

[KM] V.M. Kopytov, N.Y. Medvedev, Right-Ordered Groups, Siberian School of Algebra and Logic, Consultants Bureau, 1996.

[L] P.A. Linnell, Noncommutative localization in group rings, in Non-commutative localization in algebra and topology, London Math. Soc. Lecture Note Ser., 330 (2006), 40-59.

[N] A. Navas, On the dynamics of (left) orderable groups, Annales de l'institut Fourier 60(2010), no. $5,1685-1740$.

$[\mathrm{Ne}] \quad$ B. H. Neumann, On ordered division rings, Trans. Amer. Math. Soc. 66 (1949), 202-252.

[Pa] D.S. Passman, Infinite Crossed Products, Pure and Applied Mathematics 135, Academic Press, 1996.

[RR] A. Rhemtulla, D. Rolfsen, Local indicability in ordered groups: braids and elementary amenable groups, Proc. Amer. Math. Soc. 130 (2002), no. 9, 2569-2577.

[S] M. Simon, Stetige und v-verträgliche Automorphismen, Diplomarbeit, Universität Potsdam, 2009 .

Universität Potsdam, Institut für Mathematik, Karl-Liebknecht-Strasse 24-25, D14476 Potsdam OT Golm, Germany

E-mail address: graeter@uni-potsdam.de 